Article

\title{
The Third Spectrum of Indium: In III
}

\author{
Swapnil and Tauheed Ahmad *
}

Physics Department, Aligarh Muslim University, Aligarh 202002, India; swapnilamu@gmail.com

* Correspondence: ahmadtauheed@rediffmail.com; Tel.: +91-9837-404-077

Academic Editor: Joseph Reader

Received: 1 February 2017; Accepted: 5 June 2017; Published: 13 June 2017

\begin{abstract}
The present investigation reports on the extended study of the third spectrum of indium (In III). This spectrum was previously analyzed in many articles, but, nevertheless, this study represents a significant extension of the previous analyses. The main new contribution is connected to the observation of transitions involving core-excited configurations. Previous data are critically evaluated and in some cases are corrected. The spectra were recorded on 3-m as well as on 10.7-m normal incidence spectrographs using a triggered spark source. Theoretical calculations were made with Cowan's code. The analysis results in the identifications of 70 spectral lines and determination of 24 new energy levels. In addition, the manuscript represents a compilation of all presently available data on In III.
\end{abstract}

Keywords: spectra; ionized atoms; wavelengths; energy levels; ionization energies

\section{Introduction}

The third spectrum of indium (In III) belongs to the Ag I isoelectronic sequence with the ground state $[\mathrm{Kr}] 4 \mathrm{~d}^{10} 5 \mathrm{~s}^{2} \mathrm{~S}_{1 / 2}$. The outer electronic excitation gives rise to the $[\mathrm{Kr}] 4 \mathrm{~d}^{10} n \ell(n \geq 5$, for $\ell \leq 2$; $n \geq \ell+1$ otherwise) type of configurations with a simple doublet structure, while core excitation involving the configurations such as $4 d^{9} 5 s(5 p+4 f), 4 d^{9} 5 s^{2}$ and $4 d^{9} 5 p^{2}$ makes a complex three-electron system having both doublet and quartet terms.

Several authors studied the In III spectrum, and it is appropriate to summarize their work briefly. The first work on the third spectrum of indium was done by Rao et al. [1], followed by Lang [2], Douglas [3] and Nodwell [4]. Rao et al. [1] identified 12 lines in the wavelength region 2983-5918 and established 13 levels belonging to the $4 \mathrm{~d}^{10}(5 \mathrm{~s}, 6 \mathrm{~s}, 7 \mathrm{~s}, 5 \mathrm{p}, 6 \mathrm{p}, 5 \mathrm{~d}, 6 \mathrm{~d}, 4 \mathrm{f}, 5 \mathrm{f}$ and $5 \mathrm{~g})$ configurations. However, only six of those levels could be verified by later workers [2-4]. Nodwell [4] studied the indium spectrum in more detail. He recorded the indium spectra on a 2-m vacuum grating spectrograph and identified 56 lines of In III in the wavelength region 685-6198 А. He established 27 energy levels including six doubtful. This work is listed in the Atomic Energy Levels (AEL) compilation [5]. Bhatia [6] investigated the In III spectrum more comprehensively using a 3-m normal incidence vacuum spectrograph in the range $340-2300 \AA$ with a 1200 lines/mm grating giving a reciprocal dispersion of $2.775 \AA / \mathrm{mm}$ and a prism spectrograph in the region $2300 \AA$ to $9500 \AA$ with a disruptive electrodeless discharge. He revised and extended the earlier analysis and established the levels of the $4 \mathrm{~d}^{10} n \mathrm{~s}(n=5-12), 4 \mathrm{~d}^{10} n \mathrm{p}(n=5-9), 4 \mathrm{~d}^{10} n \mathrm{~d}(n=5-9), 4 \mathrm{~d}^{10} n \mathrm{f}(n=4-7), 4 \mathrm{~d}^{10} n \mathrm{~g}(n=5-9)$, $4 \mathrm{~d}^{10} n \mathrm{~h}(n=6-9), 4 \mathrm{~d}^{9} 5 \mathrm{~s}^{2}$, and $4 \mathrm{~d}^{9} 5 \mathrm{~s} 5 \mathrm{p}$ configurations. Kaufman et al. [7] studied the core-excited transition array $4 d^{10} 5 s-4 d^{9} 5 s 5 p$ in the isoelectronic sequence from In III to Te VI. The spectra were recorded on 10.7-m normal and grazing incidence spectrographs using a sliding spark source. Out of 23 possible levels of the $4 d^{9} 5 \mathrm{~s} 5 \mathrm{p}$ configuration, they reported only 10 that can combine with the ground level $4 \mathrm{~d}^{10} 5 \mathrm{~s}^{2} \mathrm{~S}_{1 / 2}$. Kilbane et al. [8] studied photoabsorption spectra of In II-IV with a dual laser plasma (DLP) technique. They reported the $4 \mathrm{~d}^{10} 5 \mathrm{~s}-\left\{4 \mathrm{~d}^{9} 5 \mathrm{~s} n \mathrm{p}(n=6-11)+4 \mathrm{~d}^{9} 5 \mathrm{~s} n \mathrm{f}(n=4-11)\right\}$ transition array. They could not observe the $4 d^{10} 5 s-4 d^{9} 5 s 5 p$ transitions as they lie beyond the region 
of their investigation. Recently, Ryabtsev et al. [9] added a new configuration $4 d^{9} 5 p^{2}$ to the In III-Te VI sequence and observed the $4 \mathrm{~d}^{10} 5 \mathrm{p}-4 \mathrm{~d}^{9} 5 \mathrm{p}^{2}$ transition array in the range $250-600 \AA$ using a $6.65-\mathrm{m}$ normal incidence spectrograph equipped with a 1200 lines $/ \mathrm{mm}$ grating giving a reciprocal linear dispersion of $1.25 \AA / \mathrm{mm}$. They were able to determine only 13 levels out of 28 levels of the $4 \mathrm{~d}^{9} 5 \mathrm{p}^{2}$ configuration. Skočić et al. [10] studied Stark shifts of some prominent lines of In III (6s-6p, 6p-6d, and $4 \mathrm{f}-5 \mathrm{~d})$.

As mentioned above, a number of publications on In III appeared in the literature [1-11]. Among these, Bhatia's [6] analysis was the most comprehensive and contained a large number of one-electron configurations. However, after careful examination of these results, a number of irregularities were noticed in Bhatia's results, for example, many lines classified did not match the In III characteristics on our recorded spectra and 17 reported lines have incorrect conversion between wavenumbers and wavelengths. Moreover, the levels of the $4 \mathrm{~d}^{9} 5 \mathrm{~s} 5 \mathrm{p}$ configuration reported by Kaufman et al. [7] and the levels of $4 d^{9} 5 p^{2}$ configuration established by Ryabtsev et al. [9] are still incomplete. These facts prompted us to re-investigate the In III spectrum in detail. A Grotrian energy level diagram of In III is illustrated in Figure 1 showing the basic configurations and possible transition between them.

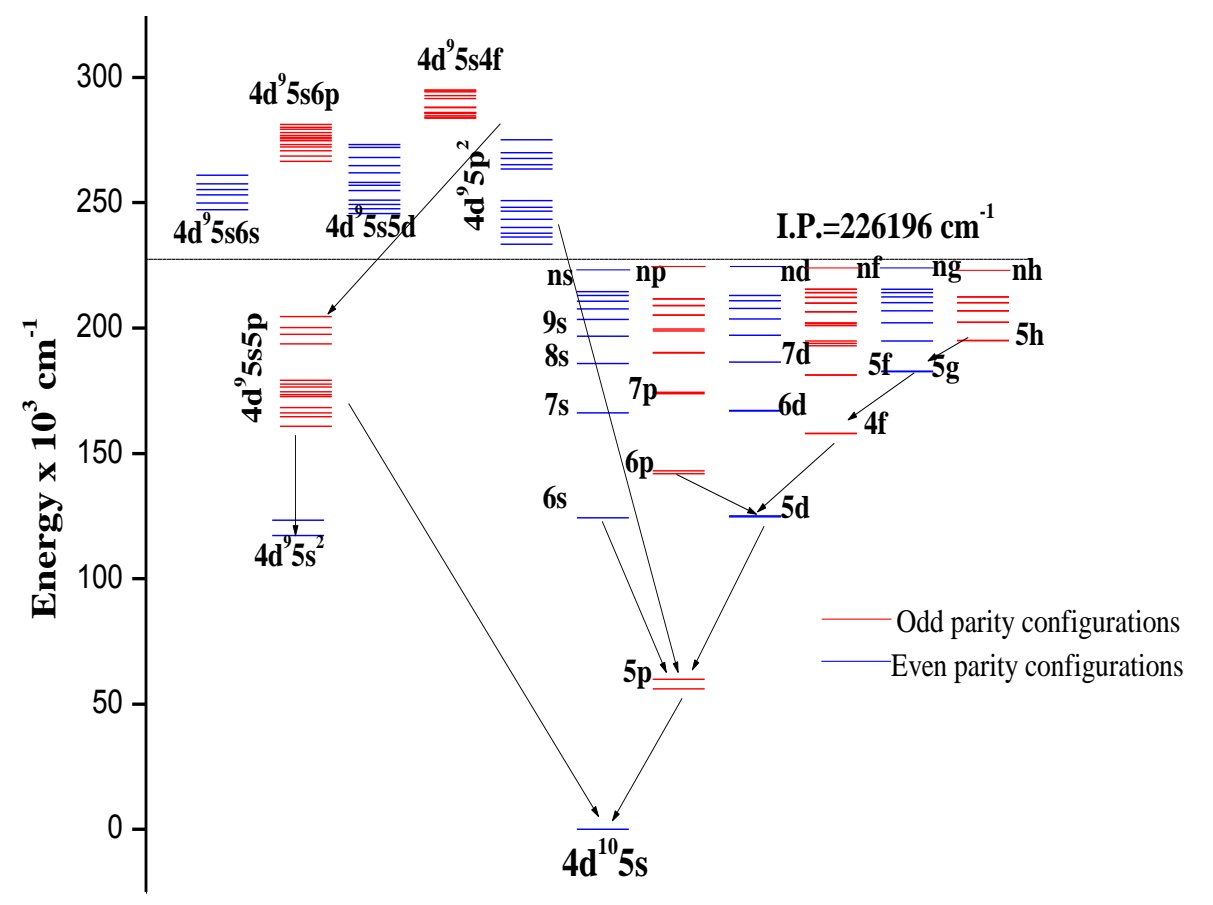

Figure 1. Grotrian diagram of In III. "I.P." denotes the ionization potential (see Section 6). Arrows denote the observed transition arrays.

\section{Experiment Detail}

The spectra were recorded at two different places. A 3-m vacuum spectrograph equipped with 2400 lines per mm holographic grating was employed at Antigonish laboratory in Nova Scotia, Canada with a triggered spark source to cover the wavelength region 350-2080 $\AA$. This spectrograph gives the first order inverse dispersion of $1.385 \AA / \mathrm{mm}$. For ionization separation of the spectral lines, either the charging potential of the source was varied or an inductance with a varying number of turns was inserted in series in the circuit. The charging unit was a $14.5 \mu \mathrm{F}$ low inductance fast charging capacitor and the charging potential was varied between 2 and 6 kV. Y.N. Joshi of St. Francis Xavier University, Antigonish (Canada) provided the indium spectra that were recorded on the 10.7-m normal incidence vacuum spectrograph of the National Institute of Standards and Technology (NIST) also using a triggered spark source. The NIST spectrograph was equipped with 1200 lines/mm grating with 
an inverse dispersion $0.78 \AA / \mathrm{mm}$. The spectrograms were measured either on an Abbec comparator at Aligarh or on a semi-automatic Grant's comparator in Antigonish, Canada. Known standard lines of oxygen, carbon, aluminium and silicon [11] were used as internal standards for the calibration of wavelengths. We estimated our measurements uncertainty for sharp and unblended lines to be within $\pm 0.006 \AA$ for wavelength below $900 \AA$ and $\pm 0.008 \AA$ above that.

\section{Theoretical Calculations}

The ab initio calculations were performed by the Hartree-Fock method with relativistic corrections using Cowan code [12] with superposition of configurations including $4 \mathrm{~d}^{10} n \mathrm{~s}(n=5-12), 4 \mathrm{~d}^{10} n \mathrm{~d}$ $(n=5-9), 4 \mathrm{~d}^{10} n \mathrm{~g}(n=5-9), 4 \mathrm{~d}^{9}\left(5 \mathrm{~s}^{2}+5 \mathrm{p}^{2}\right), 4 \mathrm{~d}^{9} 5 \mathrm{~s}(5 \mathrm{~d}+6 \mathrm{~s})$ configurations for the even parity system and $4 \mathrm{~d}^{10} n \mathrm{p}(n=5-9), 4 \mathrm{~d}^{10} n \mathrm{f}(n=4-7), 4 \mathrm{~d}^{10} n \mathrm{~h}(n=6-9), 4 \mathrm{~d}^{9} 5 \mathrm{~s} n \mathrm{p}(n=5-11), 4 \mathrm{~d}^{9} 5 \mathrm{~s} n \mathrm{f}(n=4-12), 4 \mathrm{~d}^{8} 5 \mathrm{~s}^{2} 5 \mathrm{p}$ for the odd parity matrix involving a total of 52 configurations in our calculations. The initial scaling of the Slater energy parameters was kept at $100 \%$ of the Hartree-Fock values for $E_{a v}$ and $\zeta_{n l}, 85 \%$ for $F^{k}$, and $80 \%$ for the $G^{k}$ as well as $R^{k}$ integrals. These parameters were more refined at a later stage as least squares fitted parametric calculations were performed. The main output from these programs includes the values of energy levels, wavelengths, weighted transition rates and weighted oscillator strengths. The transition probability of lines depends on the line strength and is greatly affected by the cancellation factor [13], is also calculated by Cowan's code programs [12]. The Hartree-Fock (HFR) and least-squares-fitted (LSF) energy parameters used in the present calculations are given in Table 1 along with their scaling factor (ratio of the LSF value to the HFR value) of the parameters. The standard deviations for the even and odd parity systems are $172 \mathrm{~cm}^{-1}$ and $216 \mathrm{~cm}^{-1}$, respectively.

Table 1. Least Square Fitted (LSF) Energy Parameters (in $\mathrm{cm}^{-1}$ ) for In III.

\begin{tabular}{ccccccc}
\hline Configuration & Parameters ${ }^{\mathbf{a}}$ & LSF & STD $^{\#}$ & Group $^{\mathbf{b}}$ & HFR & LSF/HFR \\
\hline \multicolumn{2}{c}{ Even Parity } & & & & & \\
\hline $5 \mathrm{~s}$ & $E_{\mathrm{av}}$ & 1525.6 & 247 & & 1560.9 & 0.9774 \\
$6 \mathrm{~s}$ & $E_{\mathrm{av}}$ & $126,947.0$ & 245 & & $124,323.4$ & 1.0211 \\
$7 \mathrm{~s}$ & $E_{\mathrm{av}}$ & $169,472.1$ & 245 & & $166,231.3$ & 1.0195 \\
$8 \mathrm{~s}$ & $E_{\mathrm{av}}$ & $189,397.9$ & 245 & & $185,887.2$ & 1.0189 \\
$9 \mathrm{~s}$ & $E_{\mathrm{av}}$ & $200,378.4$ & 245 & & $196,742.7$ & 1.0185 \\
$10 \mathrm{~s}$ & $E_{\mathrm{av}}$ & $207,041.1$ & 139 & 1 & $203,380.9$ & 1.0180 \\
$11 \mathrm{~s}$ & $E_{\mathrm{av}}$ & $211,473.3$ & 142 & 1 & $207,734.7$ & 1.0180 \\
$12 \mathrm{~s}$ & $E_{\mathrm{av}}$ & $214,537.1$ & 144 & 1 & $210,744.4$ & 1.0180 \\
$13 \mathrm{~s}$ & $E_{\mathrm{av}}$ & $216,749.1$ & 145 & 1 & $212,917.3$ & 1.0180 \\
$14 \mathrm{~s}$ & $E_{\mathrm{av}}$ & $218,395.3$ & 147 & 1 & $214,534.4$ & 1.0180 \\
$5 \mathrm{~d}$ & $E_{\mathrm{av}}$ & $128,785.8$ & 179 & & $124,706.2$ & 1.0327 \\
& $\zeta(5 \mathrm{~d})$ & 149.6 & 125 & 3 & 120.5 & 1.2415 \\
$6 \mathrm{~d}$ & $E_{\mathrm{av}}$ & $170,730.6$ & 174 & & $167,143.9$ & 1.0215 \\
& $\zeta(6 \mathrm{~d})$ & 65.7 & 55 & 3 & 52.9 & 1.2420 \\
$7 \mathrm{~d}$ & $E_{\mathrm{av}}$ & $190,146.5$ & 174 & & $186,483.2$ & 1.0196 \\
& $\zeta(7 \mathrm{~d})$ & 35.0 & 29 & 3 & 28.2 & 1.2411 \\
$8 \mathrm{~d}$ & $E_{\mathrm{av}}$ & $200,844.9$ & 174 & & $197,127.9$ & 1.0189 \\
& $\zeta(8 \mathrm{~d})$ & 20.9 & 17 & 3 & 16.8 & 1.2440 \\
$9 \mathrm{~d}$ & $E_{\mathrm{av}}$ & $207,385.2$ & 174 & 2 & $203,639.2$ & 1.0184 \\
& $\zeta(9 \mathrm{~d})$ & 13.4 & 11 & 3 & 10.8 & 1.2407 \\
$10 \mathrm{~d}$ & $E_{\mathrm{av}}$ & $211,739.0$ & 177 & 2 & $207,914.3$ & 1.0184 \\
& $\zeta(10 \mathrm{~d})$ & 9.1 & 8 & 3 & 7.3 & 1.2466 \\
$11 \mathrm{~d}$ & $E_{\mathrm{av}}$ & $214,754.5$ & 180 & 2 & $210,875.3$ & 1.0184 \\
& $\zeta(11 \mathrm{~d})$ & 6.5 & 5 & 3 & 5.2 & 1.2500 \\
$12 \mathrm{~d}$ & $E_{\mathrm{av}}$ & $216,933.8$ & 182 & 2 & $213,015.3$ & 1.0184 \\
& $\zeta(12 \mathrm{~d})$ & 4.7 & 4 & 3 & 3.8 & 1.2368 \\
$5 \mathrm{~g}$ & $E_{\mathrm{av}}$ & $186,530.4$ & 173 & & $182,689.5$ & 1.0210 \\
& $\zeta(5 \mathrm{~d})$ & 0.3 & Fixed & & 0.3 & 1.0000 \\
$6 \mathrm{~g}$ & $E_{\mathrm{av}}$ & $198,656.5$ & 173 & & $194,809.9$ & 1.0197 \\
& $\zeta(5 \mathrm{~d})$ & 0.2 & Fixed & & 0.2 & 1.0000 \\
\hline
\end{tabular}


Table 1. Cont.

\begin{tabular}{|c|c|c|c|c|c|c|}
\hline Configuration & Parameters $^{a}$ & LSF & STD $^{\#}$ & Group $^{b}$ & HFR & LSF/HFR \\
\hline \multirow[t]{2}{*}{$7 \mathrm{~g}$} & $E_{\mathrm{av}}$ & $205,968.6$ & 173 & & $202,130.8$ & 1.0190 \\
\hline & $\zeta(5 \mathrm{~d})$ & 0.1 & Fixed & & 0.1 & 1.0000 \\
\hline \multirow[t]{2}{*}{$8 g$} & $E_{\mathrm{av}}$ & $210,713.4$ & 173 & & $206,885.7$ & 1.0185 \\
\hline & $\zeta(5 \mathrm{~d})$ & 0.1 & Fixed & & 0.1 & 1.0000 \\
\hline \multirow[t]{2}{*}{$9 g$} & $E_{\mathrm{av}}$ & $213,967.7$ & 173 & 4 & $210,144.3$ & 1.0182 \\
\hline & $\zeta(5 d)$ & 0.0 & Fixed & & 0.0 & \\
\hline \multirow[t]{2}{*}{$10 \mathrm{~g}$} & $E_{\mathrm{av}}$ & $216,340.0$ & 175 & 4 & $212,474.2$ & 1.0182 \\
\hline & $\zeta(5 \mathrm{~d})$ & 0.0 & Fixed & & 0.0 & \\
\hline \multirow[t]{2}{*}{$11 \mathrm{~g}$} & $E_{\mathrm{av}}$ & $218,091.5$ & 177 & 4 & $214,194.4$ & 1.0182 \\
\hline & $\zeta(11 \mathrm{~g})$ & 0.0 & Fixed & & 0.0 & \\
\hline \multirow[t]{2}{*}{$12 \mathrm{~g}$} & $E_{\mathrm{av}}$ & $219,428.0$ & 178 & 4 & $215,507.0$ & 1.0182 \\
\hline & $\zeta(12 \mathrm{~g})$ & 0.0 & Fixed & & 0.0 & \\
\hline \multirow[t]{2}{*}{$4 d^{9} 5 s^{2}$} & $E_{\mathrm{av}}$ & $122,546.3$ & 196 & & $124,206.8$ & 0.9866 \\
\hline & $\zeta(4 \mathrm{~d})$ & 2827.6 & 53 & 5 & 2706.2 & 1.0449 \\
\hline \multirow[t]{7}{*}{$4 d^{9} 5 p^{2}$} & $E_{\mathrm{av}}$ & $248,495.0$ & 551 & & $246,591.5$ & 1.0077 \\
\hline & $F^{2}(5 p, 5 p)$ & $27,409.1$ & 4453 & & $39,408.9$ & 0.6955 \\
\hline & $\zeta(4 d)$ & 2855.5 & 54 & 5 & 2732.9 & 1.0449 \\
\hline & $\zeta(5 p)$ & 3648.0 & 138 & & 2988.1 & 1.2208 \\
\hline & $F^{2}(4 d, 5 p)$ & $20,103.9$ & 530 & & $24,204.1$ & 0.8306 \\
\hline & $G^{1}(4 d, 5 p)$ & 6453.4 & Fixed & & 7592.2 & 0.8500 \\
\hline & $G^{3}(4 d, 5 p)$ & 5725.3 & Fixed & & 6735.6 & 0.8500 \\
\hline $4 d^{9} 5 s 5 d^{*}$ & $E_{\mathrm{av}}$ & $262,301.3$ & 2850 & & $253,437.2$ & 1.0350 \\
\hline \multirow{2}{*}{$4 d^{9} 5 \mathrm{~s} 6 \mathrm{~s} *$} & $E_{\mathrm{av}}$ & $252,606.4$ & Fixed & & $252,606.4$ & 1.0000 \\
\hline & $\sigma^{\#}$ & 172 & & & & \\
\hline \multicolumn{7}{|l|}{ Odd Parity } \\
\hline \multirow[t]{2}{*}{$5 p$} & $E_{\mathrm{av}}$ & $60,352.9$ & 42 & 1 & $59,151.7$ & 1.0203 \\
\hline & $\zeta(5 p)$ & 2671.6 & 242 & 2 & 2505.1 & 1.0665 \\
\hline \multirow[t]{2}{*}{$6 p$} & $E_{\mathrm{av}}$ & $145,688.0$ & 103 & 1 & $142,788.3$ & 1.0203 \\
\hline & $\zeta(6 p)$ & 863.2 & 78 & 2 & 809.4 & 1.0665 \\
\hline \multirow[t]{2}{*}{$7 \mathrm{p}$} & $E_{\mathrm{av}}$ & $178,073.7$ & 125 & 1 & $174,529.4$ & 1.0203 \\
\hline & $\zeta(7 p)$ & 396.3 & 36 & 2 & 371.6 & 1.0665 \\
\hline \multirow[t]{2}{*}{$8 p$} & $E_{\mathrm{av}}$ & $194,213.5$ & 137 & 1 & $190,348.0$ & 1.0203 \\
\hline & $\zeta(8 p)$ & 216.2 & 20 & 2 & 202.7 & 1.0666 \\
\hline $9 p$ & $\underline{E}_{\mathrm{av}}$ & $203,466.4$ & 143 & 1 & $199,416.7$ & 1.0203 \\
\hline & $\zeta(9 p)$ & 131.0 & 12 & 2 & 122.8 & 1.0668 \\
\hline $10 p$ & $E_{\mathrm{av}}$ & $209,274.8$ & 147 & 1 & $205,109.5$ & 1.0203 \\
\hline & $\zeta(10 p)$ & 85.4 & 8 & 2 & 80.1 & 1.0662 \\
\hline $11 p$ & $E_{\mathrm{av}}$ & $213,160.3$ & 150 & 1 & $208,917.6$ & 1.0203 \\
\hline & $\zeta(11 p)$ & 58.8 & 5 & 2 & 55.1 & 1.0672 \\
\hline $12 p$ & $E_{\mathrm{av}}$ & $215,887.1$ & 152 & 1 & $211,590.2$ & 1.0203 \\
\hline & $\zeta(12 p)$ & 42.1 & 4 & 2 & 39.5 & 1.0658 \\
\hline $4 \mathrm{f}$ & $E_{\mathrm{av}}$ & $162,121.7$ & 202 & & $158,107.9$ & 1.0254 \\
\hline & $\zeta(4 \mathrm{f})$ & 1.2 & Fixed & & 1.2 & 1.0000 \\
\hline $5 f$ & $E_{\mathrm{av}}$ & $185,069.7$ & 206 & & $181,299.2$ & 1.0208 \\
\hline & $\zeta(5 f)$ & 0.8 & Fixed & & 0.8 & 1.0000 \\
\hline $6 f$ & $E_{\mathrm{av}}$ & $191,442.6$ & 406 & 3 & $193,937.2$ & 0.9871 \\
\hline & $\zeta(6 \mathrm{f})$ & 0.5 & Fixed & & 0.5 & 1.0000 \\
\hline $7 f$ & $E_{\mathrm{av}}$ & $198,958.4$ & 422 & 3 & $201,550.9$ & 0.9871 \\
\hline & $\zeta(7 \mathrm{f})$ & 0.3 & Fixed & & 0.3 & 1.0000 \\
\hline $8 \mathrm{f}$ & $E_{\mathrm{av}}$ & $203,826.5$ & 432 & 3 & $206,482.5$ & 0.9871 \\
\hline & $\zeta(8 \mathrm{f})$ & 0.2 & Fixed & & 0.2 & 1.0000 \\
\hline $9 f$ & $E_{\mathrm{av}}$ & $207,155.3$ & 439 & 3 & $209,854.6$ & 0.9871 \\
\hline & $\zeta(9 \mathrm{f})$ & 0.2 & Fixed & & 0.2 & 1.0000 \\
\hline $10 \mathrm{f}$ & $E_{\mathrm{av}}$ & $209,529.7$ & 445 & 3 & $212,260.0$ & 0.9871 \\
\hline & $\zeta(10 \mathrm{f})$ & 0.1 & Fixed & & 0.1 & 1.0000 \\
\hline $11 \mathrm{f}$ & $E_{\mathrm{av}}$ & $211,278.0$ & 448 & 3 & $214,031.1$ & 0.9871 \\
\hline & $\zeta(11 \mathrm{f})$ & 0.1 & Fixed & & 0.1 & 1.0000 \\
\hline $12 \mathrm{f}$ & $E_{\mathrm{av}}$ & $212,609.0$ & 451 & 3 & $215,379.4$ & 0.9871 \\
\hline & $\zeta(12 \mathrm{f})$ & 0.1 & Fixed & & 0.1 & 1.0000 \\
\hline $6 \mathrm{~h}$ & $E_{\mathrm{av}}$ & $198,520.8$ & 109 & 4 & $194,930.4$ & 1.0184 \\
\hline & $\zeta(6 h)$ & 0.1 & Fixed & & 0.1 & 1.0000 \\
\hline $7 \mathrm{~h}$ & $E_{\mathrm{av}}$ & $205,935.4$ & 113 & 4 & $202,210.9$ & 1.0184 \\
\hline & $\zeta(7 \mathrm{~h})$ & 0.1 & Fixed & & 0.1 & 1.0000 \\
\hline
\end{tabular}


Table 1. Cont

\begin{tabular}{ccccccc}
\hline Configuration & Parameters $^{\mathbf{a}}$ & LSF & STD $^{\#}$ & Group $^{\mathbf{b}}$ & HFR & LSF/HFR \\
\hline $8 \mathrm{~h}$ & $E_{\mathrm{av}}$ & $210,752.2$ & 116 & 4 & $206,940.6$ & 1.0184 \\
& $\zeta(8 \mathrm{~h})$ & 0.1 & Fixed & & 0.1 & 1.0000 \\
$9 \mathrm{~h}$ & $E_{\mathrm{av}}$ & $214,053.7$ & 118 & 4 & $210,182.4$ & 1.0184 \\
& $\zeta(9 \mathrm{~h})$ & 0.0 & Fixed & & 0.0 & \\
$10 \mathrm{~h}$ & $E_{\mathrm{av}}$ & $216,415.1$ & 119 & 4 & $212,501.1$ & 1.0184 \\
& $\zeta(10 \mathrm{~h})$ & 0.0 & Fixed & & 0.0 & \\
$4 \mathrm{~d}^{9} 5 \mathrm{~s} 5 \mathrm{p}$ & $E_{\mathrm{av}}$ & $179,088.7$ & 71 & & $177,339.0$ & 1.0099 \\
& $\zeta(4 \mathrm{~d})$ & 2747.9 & 79 & & 2718.8 & 1.0107 \\
& $\zeta(5 \mathrm{p})$ & 3591.3 & 175 & & 2994.7 & 1.1992 \\
& $F^{2}(4 \mathrm{~d}, 5 \mathrm{p})$ & $196,94.2$ & 839 & & $24,199.1$ & 0.8138 \\
& $G^{2}(4 \mathrm{~d}, 5 \mathrm{~s})$ & $12,656.6$ & 1434 & & $13,315.8$ & 0.9505 \\
& $G^{1}(4 \mathrm{~d}, 5 \mathrm{p})$ & 7671.9 & 563 & 5 & 7655.9 & 1.0021 \\
& $G^{3}(4 \mathrm{~d}, 5 \mathrm{p})$ & 6794.6 & 499 & 5 & 6780.4 & 1.0021 \\
& $G^{1}(5 \mathrm{~s}, 5 \mathrm{p})$ & $33,087.5$ & 270 & & $48,386.8$ & 0.6838 \\
$4 \mathrm{~d}^{9} 5 \mathrm{~s} 6 \mathrm{p}$ & $E_{\mathrm{av}}$ & $275,446.9$ & 127 & & $272,620.0$ & 1.0104 \\
& $\sigma^{\#}$ & 216 & & & & \\
\hline
\end{tabular}

a All configuration-interaction parameters $R^{\mathrm{k}}$ for even and odd parity configurations were fixed at $80 \%$ of the Hartree-Fock value. ${ }^{b}$ Parameters in each numbered group were linked together with their ratio fixed at the Hartree-Fock level. " $\sigma$ and STD are the standard deviations of the fit for the levels and parameters, respectively.

* Only $E_{\mathrm{av}}$ of unobserved interacting configurations are given.

\section{Spectrum Analysis}

The initial approach of the analysis was to identify In III lines with correct ionization character. A computer code FIND3 [14] was useful in the analysis to search for new levels. A total of 91 levels have been established, of which 24 are new; they are assembled in Table 2 along with least squares fitted values and LS percentage composition. Two hundred fifty-one lines have been classified in In III and they are given in Table 3 along with their transition probabilities. In the present analysis, apart from the one- electron spectrum $4 \mathrm{~d}^{10} n \ell$, the configurations involving inner-shell excitation, such as $4 d^{9} 5 s(5 p+4 f), 4 d^{9} 5 s^{2}$ and $4 d^{9} 5 p^{2}$ have also been studied extensively. The following sections describe them in detail. 
Table 2. Optimized energy levels of in III.

\begin{tabular}{|c|c|c|c|c|c|c|c|c|c|c|c|c|}
\hline \multirow{3}{*}{$\begin{array}{c}J \\
\text { Even Parity }\end{array}$} & \multicolumn{9}{|c|}{ LS Compositions ${ }^{\mathrm{d}}$} & \multirow{3}{*}{$\begin{array}{c}\text { 3rd } \\
\text { Component }\end{array}$} & \multirow[b]{2}{*}{ No. of Lines ${ }^{e}$} & \multirow[b]{2}{*}{ Lev. Ref. ${ }^{f}$} \\
\hline & Energy $^{a} \mathrm{~cm}^{-1}$ & $\mathrm{Unc}^{\mathrm{b}}$ & $\Delta E \mathrm{o}-\mathrm{c}^{\mathrm{c}} \mathrm{cm}^{-1}$ & & $\begin{array}{c}1 \text { st } \\
\text { Component }\end{array}$ & & & & $\begin{array}{c}\text { 2nd } \\
\text { Component }\end{array}$ & & & \\
\hline & & & & & & & & & & & & \\
\hline 0.5 & 0.00 & 0.3 & 0 & 99 & $4 d^{10} 5 s$ & ${ }^{2} \mathrm{~S}$ & & & & & 18 & B * \\
\hline 2.5 & $115,572.19$ & 0.25 & 71 & 97 & $4 d^{9} 5 s^{2}$ & ${ }^{2} \mathrm{D}$ & 3 & $4 d^{9} 5 p^{2}\left({ }^{1} S\right)$ & ${ }^{2} \mathrm{D}$ & & 9 & B* \\
\hline 1.5 & $122,419.73$ & 0.22 & -74 & 95 & $4 d^{9} 5 s^{2}$ & ${ }^{2} \mathrm{D}$ & 3 & $4 d^{9} 5 p^{2}\left({ }^{1} s\right)$ & ${ }^{2} \mathrm{D}$ & & 16 & $\mathrm{~B}^{*}$ \\
\hline 0.5 & $126,879.89$ & 0.24 & 0 & 100 & $4 \mathrm{~d}^{10} 6 \mathrm{~s}$ & ${ }^{2} \mathrm{~S}$ & & & & & 7 & B* \\
\hline 1.5 & $128,458.36$ & 0.23 & 6 & 97 & $4 d^{10} 5 d$ & ${ }^{2} \mathrm{D}$ & 2 & $4 d^{9} 5 s^{2}$ & ${ }^{2} \mathrm{D}$ & & 14 & B* \\
\hline 2.5 & $128,748.33$ & 0.25 & -6 & 99 & $4 d^{10} 5 \mathrm{~d}$ & ${ }^{2} \mathrm{D}$ & & & & & 10 & B* \\
\hline 0.5 & $169,434.59$ & 0.25 & 0 & 100 & $4 \mathrm{~d}^{10} 7 \mathrm{~s}$ & ${ }^{2} \mathrm{~S}$ & & & & & 3 & B * \\
\hline 1.5 & $170,535.76$ & 0.24 & -8 & 100 & $4 d^{10} 6 \mathrm{~d}$ & ${ }^{2} \mathrm{D}$ & & & & & 4 & B * \\
\hline 2.5 & $170,718.81$ & 0.3 & 8 & 100 & $4 d^{10} 6 \mathrm{~d}$ & ${ }^{2} \mathrm{D}$ & & & & & 2 & B* \\
\hline 3.5 & $186,527.40$ & 0.3 & 0 & 100 & $4 d^{10} 5 g$ & ${ }^{2} \mathrm{G}$ & & & & & 4 & B * \\
\hline 4.5 & $186,528.26$ & 0.3 & -1 & 100 & $4 d^{10} 5 \mathrm{~g}$ & ${ }^{2} \mathrm{G}$ & & & & & 4 & B * \\
\hline 0.5 & $189,374.5$ & 0.3 & 1 & 100 & $4 \mathrm{~d}^{10} 8 \mathrm{~s}$ & ${ }^{2} \mathrm{~S}$ & & & & & 4 & B * \\
\hline 1.5 & $190,038.8$ & 0.3 & -4 & 100 & $4 d^{10} 7 d$ & ${ }^{2} \mathrm{D}$ & & & & & 4 & B* \\
\hline 2.5 & $190,136.3$ & 0.4 & 4 & 100 & $4 d^{10} 7 d$ & ${ }^{2} \mathrm{D}$ & & & & & 2 & B* \\
\hline 4.5 & $198,654.0$ & 0.8 & 0 & 100 & $4 d^{10} 6 g$ & ${ }^{2} \mathrm{G}$ & & & & & 1 & B * \\
\hline 3.5 & $198,654.3$ & 0.4 & 0 & 100 & $4 d^{10} 6 \mathrm{~g}$ & ${ }^{2} \mathrm{G}$ & & & & & 2 & $\mathrm{~B}^{*}$ \\
\hline 0.5 & $200,362.77$ & 0.3 & 0 & 100 & $4 d^{10} 9 \mathrm{~s}$ & ${ }^{2} \mathrm{~S}$ & & & & & 4 & B* \\
\hline 1.5 & $200,778.32$ & 0.23 & -2 & 100 & $4 d^{10} 8 d$ & ${ }^{2} \mathrm{D}$ & & & & & 5 & B* \\
\hline 2.5 & $200,836.01$ & 0.24 & 2 & 100 & $4 d^{10} 8 d$ & ${ }^{2} \mathrm{D}$ & & & & & 3 & B* \\
\hline 3.5 & $205,966.56$ & 0.3 & 1 & 100 & $4 d^{10} 7 g$ & ${ }^{2} \mathrm{G}$ & & & & & 3 & B * \\
\hline 4.5 & $205,966.76$ & 0.3 & 0 & 100 & $4 d^{10} 7 \mathrm{~g}$ & ${ }^{2} \mathrm{G}$ & & & & & 2 & $\mathrm{~B}^{*}$ \\
\hline 0.5 & $207,068.43$ & 0.3 & 38 & 100 & $4 \mathrm{~d}^{10} 10 \mathrm{~s}$ & ${ }^{2} \mathrm{~S}$ & & & & & 4 & B* \\
\hline 1.5 & $207,338.8$ & 0.4 & -3 & 100 & $4 d^{10} 9 d$ & ${ }^{2} \mathrm{D}$ & & & & & 3 & B* \\
\hline 2.5 & $207,379.7$ & 0.4 & 3 & 100 & $4 d^{10} 9 d$ & ${ }^{2} \mathrm{D}$ & & & & & 2 & B * \\
\hline 4.5 & $210,710.88$ & 0.3 & -1 & 100 & $4 d^{10} 8 g$ & ${ }^{2} \mathrm{G}$ & & & & & 1 & B* \\
\hline 3.5 & $210,713.04$ & 0.3 & 1 & 100 & $4 d^{10} 8 g$ & ${ }^{2} \mathrm{G}$ & & & & & 3 & B * \\
\hline 0.5 & $211,462.1$ & 0.3 & -3 & 100 & $4 \mathrm{~d}^{10} 11 \mathrm{~s}$ & ${ }^{2} \mathrm{~S}$ & & & & & 4 & B * \\
\hline 1.5 & $(211,708.6)$ & & & 100 & $4 d^{10} 10 d$ & ${ }^{2} \mathrm{D}$ & & & & & & \\
\hline 2.5 & $(211,732.5)$ & & & 100 & $4 \mathrm{~d}^{10} 10 \mathrm{~d}$ & ${ }^{2} \mathrm{D}$ & & & & & & \\
\hline 4.5 & $213,966.18$ & 0.3 & -1 & 100 & $4 d^{10} 9 g$ & ${ }^{2} \mathrm{G}$ & & & & & 2 & B * \\
\hline 3.5 & $213,966.94$ & 0.4 & 1 & 100 & $4 d^{10} 9 g$ & ${ }^{2} \mathrm{G}$ & & & & & 1 & B * \\
\hline 0.5 & $214,497.7$ & 1.4 & -33 & 100 & $4 d^{10} 12 s$ & ${ }^{2} \mathrm{~S}$ & & & & & 2 & B* \\
\hline 1.5 & $(214,732.2)$ & & & 100 & $4 d^{10} 11 d$ & ${ }^{2} \mathrm{D}$ & & & & & & \\
\hline 2.5 & $(214,749.3)$ & & & 100 & $4 d^{10} 11 d$ & ${ }^{2} \mathrm{D}$ & & & & & & \\
\hline 3.5 & $(216,339)$ & & & 100 & $4 \mathrm{~d}^{10} 10 \mathrm{~g}$ & ${ }^{2} \mathrm{G}$ & & & & & & \\
\hline 4.5 & $(216,339.2)$ & & & 100 & $4 d^{10} 10 \mathrm{~g}$ & ${ }^{2} \mathrm{G}$ & & & & & & \\
\hline 0.5 & $(216,744.7)$ & & & 100 & $4 d^{10} 13 \mathrm{~s}$ & ${ }^{2} \mathrm{~S}$ & & & & & & \\
\hline 1.5 & $(216,917.2)$ & & & 100 & $4 d^{10} 12 d$ & ${ }^{2} \mathrm{D}$ & & & & & & \\
\hline
\end{tabular}


Table 2. Cont.

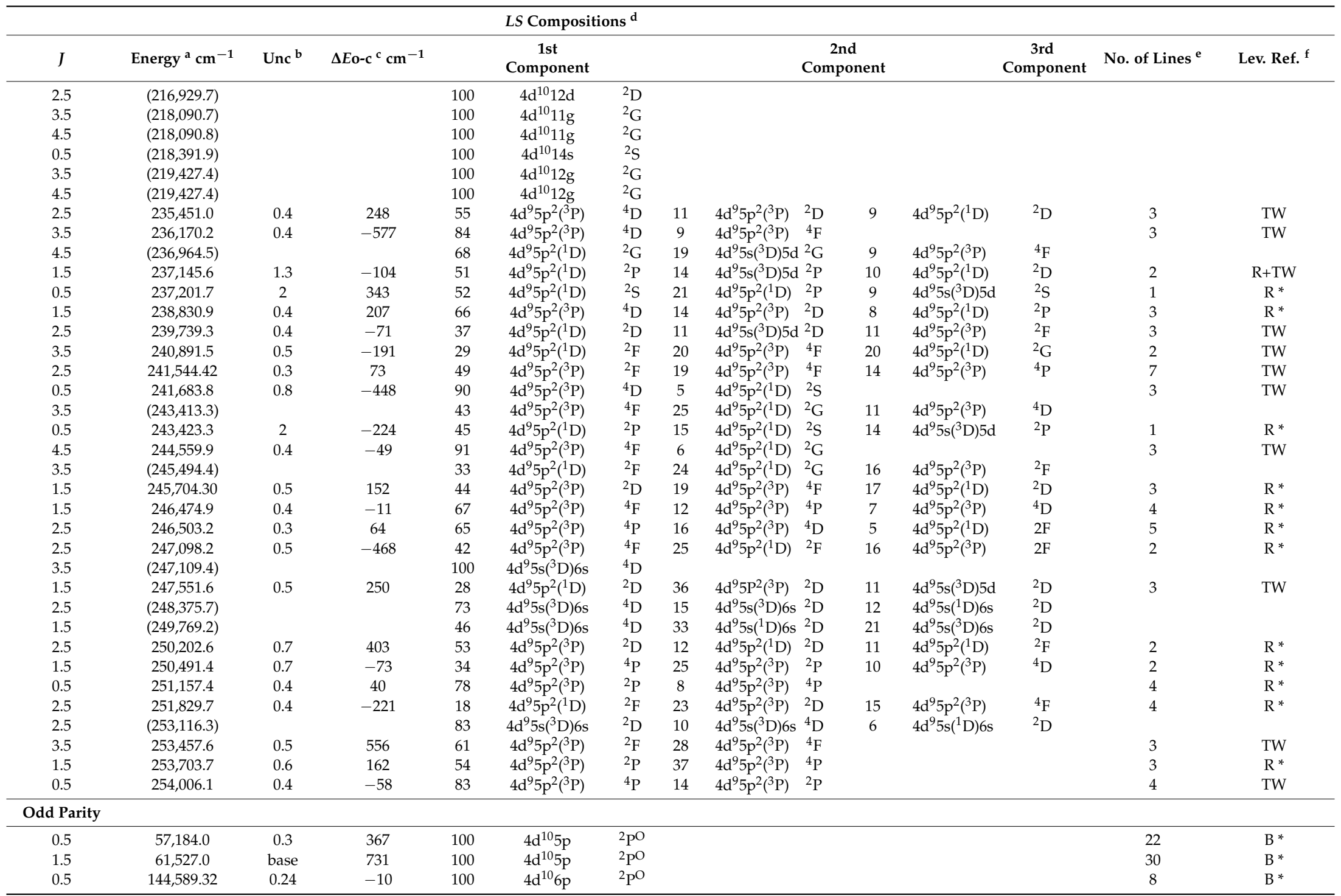


Table 2. Cont.

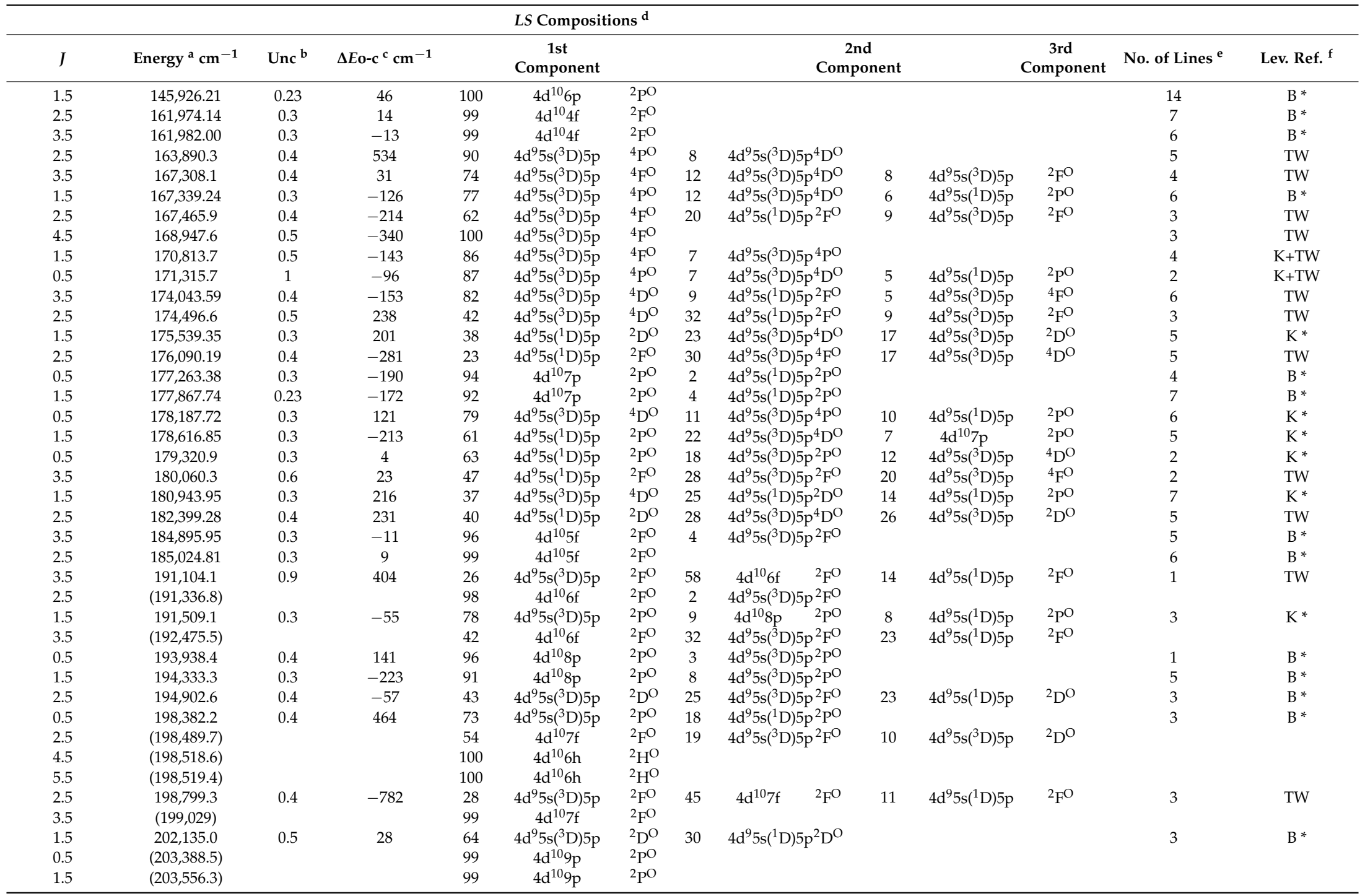


Table 2. Cont.

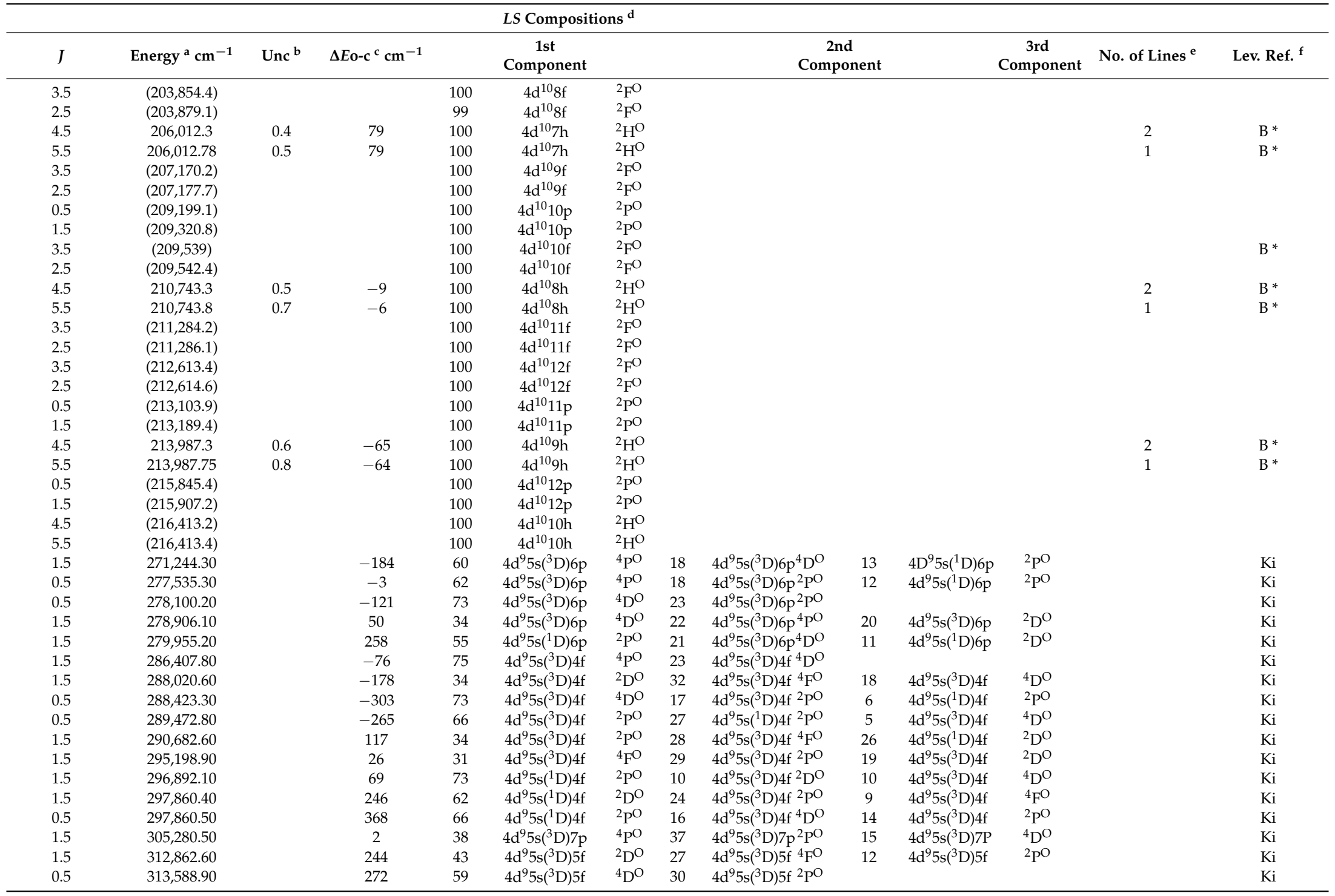


Table 2. Cont.

\begin{tabular}{|c|c|c|c|c|c|c|c|c|c|c|c|c|c|}
\hline \multirow{3}{*}{$J$} & \multirow{3}{*}{$\begin{array}{c}\text { Energy }^{\mathbf{a}} \mathbf{c m}^{-1} \\
314,636.90\end{array}$} & \multirow[b]{2}{*}{ Unc $^{b}$} & \multicolumn{8}{|c|}{ LS Compositions ${ }^{\mathrm{d}}$} & \multirow[b]{2}{*}{$\begin{array}{c}\text { 3rd } \\
\text { Component }\end{array}$} & \multirow[b]{2}{*}{ No. of Lines ${ }^{e}$} & \multirow[b]{2}{*}{ Lev. Ref. ${ }^{\mathrm{f}}$} \\
\hline & & & $\Delta E \mathrm{o}-\mathrm{c}^{\mathrm{c}} \mathrm{cm}^{-1}$ & & $\begin{array}{c}\text { 1st } \\
\text { Component }\end{array}$ & & & $\begin{array}{r}2 \\
\mathrm{Com}_{1}\end{array}$ & nent & & & & \\
\hline & & & -317 & 29 & $4 d^{9} 5 s\left({ }^{3} D\right) 5 f$ & ${ }^{2} \mathrm{P}^{\mathrm{O}}$ & 26 & $4 d^{9} 5 s\left({ }^{3} D\right) 5 f{ }^{4} F^{O}$ & 23 & $4 d^{9} 5 s\left({ }^{1} D\right) 5 f$ & ${ }^{2} \mathrm{D}^{\mathrm{O}}$ & & $\mathrm{Ki}$ \\
\hline 1.5 & $321,653.00$ & & 116 & 70 & $4 d^{9} 5 s\left({ }^{1} D\right) 5 f$ & ${ }^{2} \mathrm{P}^{\mathrm{O}}$ & 9 & $4 d^{9} 5 s\left({ }^{3} D\right) 5 f^{2} D^{O}$ & 9 & $4 d^{9} 5 s\left({ }^{3} D\right) 5 f$ & ${ }^{4} \mathrm{D}^{\mathrm{O}}$ & & $\mathrm{Ki}$ \\
\hline 0.5 & $321,653.40$ & & -352 & 61 & $4 d^{9} 5 s\left({ }^{1} D\right) 5 f$ & ${ }^{2} \mathrm{P}^{\mathrm{O}}$ & 15 & $4 d^{9} 5 s\left({ }^{3} D\right) 5 f^{4} D^{O}$ & 10 & $4 d^{9} 5 s\left({ }^{3} D\right) 5 f$ & ${ }^{2} \mathrm{P}^{\mathrm{O}}$ & & $\mathrm{Ki}$ \\
\hline 1.5 & $325,283.70$ & & -311 & 48 & $4 d^{9} 5 s\left({ }^{3} D\right) 6 f$ & ${ }^{2} \mathrm{D}^{\mathrm{O}}$ & 22 & $\left.4 d^{9} 5 s^{3} D\right) 6 f^{4} F^{O}$ & 14 & $4 d^{9} 5 s\left({ }^{3} D\right) 6 f$ & ${ }^{2} \mathrm{PO}$ & & $\mathrm{Ki}$ \\
\hline 0.5 & $326,170.10$ & & -153 & 39 & $4 d^{9} 5 s\left({ }^{3} D\right) 6 f$ & ${ }^{4} \mathrm{D}^{\mathrm{O}}$ & 36 & $4 \mathrm{~d}^{9} 5 \mathrm{~s}\left({ }^{3} \mathrm{D}\right) 6 \mathrm{f}^{2} \mathrm{P}^{\mathrm{O}}$ & 7 & $4 d^{9} 5 s\left({ }^{3} \mathrm{D}\right) 8 \mathrm{P}$ & ${ }^{2} \mathrm{PO}$ & & $\mathrm{Ki}$ \\
\hline 1.5 & $328,106.90$ & & 348 & 26 & $4 d^{9} 5 s\left({ }^{3} D\right) 6 f$ & ${ }^{2} \mathrm{P}^{\mathrm{O}}$ & 23 & $4 d^{9} 5 s\left({ }^{3} D\right) 6 f^{4} \mathrm{~F}^{O}$ & 18 & $4 d^{9} 5 s\left({ }^{1} D\right) 6 f$ & ${ }^{2} \mathrm{D}^{\mathrm{O}}$ & & $\mathrm{Ki}$ \\
\hline 1.5 & $332,461.90$ & & 162 & 28 & $4 d^{9} 5 s\left({ }^{3} D\right) 6 f$ & ${ }^{2} \mathrm{PO}^{\mathrm{O}}$ & 23 & $4 \mathrm{~d}^{9} 5 \mathrm{~s}\left({ }^{3} \mathrm{D}\right) 6 \mathrm{f}^{4} \mathrm{~F}^{\mathrm{O}}$ & 19 & $4 d^{9} 5 s\left({ }^{3} D\right) 6 f$ & ${ }^{2} \mathrm{D}^{\mathrm{O}}$ & & $\mathrm{Ki}$ \\
\hline 1.5 & $333,429.00$ & & 42 & 46 & $4 d^{9} 5 s\left({ }^{3} D\right) 7 f$ & ${ }^{2} \mathrm{D}^{\mathrm{O}}$ & 17 & $4 \mathrm{~d}^{9} 5 \mathrm{~s}\left({ }^{3} \mathrm{D}\right) 7 \mathrm{f}^{4} \mathrm{~F}^{\mathrm{O}}$ & 16 & $4 d^{9} 5 s\left({ }^{3} D\right) 7 f$ & ${ }^{2} \mathrm{P}^{\mathrm{O}}$ & & $\mathrm{Ki}$ \\
\hline 0.5 & $333,429.80$ & & -63 & 59 & $4 d^{9} 5 s\left({ }^{3} D\right) 7 f$ & ${ }^{2} \mathrm{PO}^{\mathrm{O}}$ & 23 & $4 d^{9} 5 s\left({ }^{3} D\right) 7 f^{4} D^{O}$ & 10 & $4 d^{9} 5 s\left({ }^{1} \mathrm{D}\right) 6 \mathrm{f}$ & ${ }^{2} \mathrm{PO}$ & & $\mathrm{Ki}$ \\
\hline
\end{tabular}

a Energy values are optimized from observed wavelengths using the least squares level optimization code LOPT [15]. Values enclosed in parentheses correspond to unobserved energy levels found from the parametric least squares fitting. ${ }^{\mathrm{b}}$ Uncertainties resulting from the level optimization procedure are given on the level of one standard deviation. They correspond to uncertainties of level separations from $4 \mathrm{~d}^{10} 5 \mathrm{p}^{2} \mathrm{P}_{3 / 2}$. To determine uncertainties of excitation energies from the ground level, the given values should be combined in quadrature with the uncertainty of the ground level, $0.3 \mathrm{~cm}^{-1}$. If this column is blank, the level value was not included in the level optimization. ${ }^{\mathrm{c}}$ Differences between observed energies and those calculated in the parametric least squares fitting. ${ }^{\mathrm{d}}$ Only three leading $L S$ components are given. ${ }^{\mathrm{e}}$ Number of observed lines determining the level in the optimization procedure LOPT [15]. ${ }^{\mathrm{f}}$ Reference to the level source as B, K, R, K+TW, R+TW and TW stand for Bhatia [6], Kaufman et al. [7], Ryabtsev et al. [9], previous value [7] has been revised, previous value [9] has been revised, and this work ; * stands for levels from [6,7,9] re-optimized in this work. Ki stands for Kilbane et al. [8] level values, which have not been included in the level optimization.

Table 3. List of classified lines in In III spectrum.

\begin{tabular}{|c|c|c|c|c|c|c|c|c|c|c|c|c|c|c|c|}
\hline$I_{\text {obs }}{ }^{a}$ & $\mathrm{ch}^{\mathrm{b}}$ & $\lambda_{\text {obs }}{ }^{\mathrm{c}} \AA$ & $\sigma_{\mathrm{obs}} \mathrm{cm}^{-1}$ & $\lambda_{\text {Ritz }}{ }^{\mathrm{d}} \AA$ & $\Delta \lambda_{\text {O-Ritz }}{ }^{\mathrm{e}} \AA$ & & Classification $^{\mathrm{f}}$ & & & & $E_{\text {low }} \mathrm{cm}^{-1}$ & $E_{\mathrm{upp}} \mathrm{cm}^{-1}$ & $g A^{\mathrm{h}} \mathrm{S}^{-1}$ & & Lin. $\operatorname{Ref}^{\mathrm{i}}$ \\
\hline 50 & & $494.715(8)$ & 202,137 & 494.7189(13) & -0.004 & $4 d^{10} 5 \mathrm{~s}$ & $\left({ }^{1} \mathrm{~S}\right)^{2} \mathrm{~S}_{0.5}$ & - & $4 d^{9} 5 \mathrm{~s} 5 p$ & $\left({ }^{3} \mathrm{D}\right)^{2} \mathrm{D}_{1.5}$ & 0.0 & $202,135.0$ & $1.42 \mathrm{E}+08$ & \# & TW \\
\hline 200 & & $504.080(6)$ & $198,381.2$ & $504.0775(12)$ & 0.003 & $4 d^{10} 5 \mathrm{~s}$ & $\left({ }^{1} \mathrm{~S}\right)^{2} \mathrm{~S}_{0.5}$ & - & $4 d^{9} 5 s 5 p$ & $\left({ }^{3} \mathrm{D}\right)^{2} \mathrm{P}_{0.5}$ & 0.0 & $198,382.2$ & $5.08 \mathrm{E}+09$ & & TW \\
\hline 50 & & $508.066(6)$ & $196,824.8$ & $508.0730(12)$ & -0.007 & $4 d^{10} 5 p$ & $\left({ }^{1} \mathrm{~S}\right)^{2} \mathrm{P}_{0.5}$ & - & $4 d^{9} 5 p^{2}$ & $\left({ }^{3} \mathrm{P}\right)^{4} \mathrm{P}_{0.5}$ & $57,184.0$ & $254,006.1$ & $8.17 \mathrm{E}+08$ & & TW \\
\hline 100 & & $508.846(6)$ & $196,523.1$ & $508.8548(15)$ & -0.009 & $4 d^{10} 5 p$ & $\left({ }^{1} \mathrm{~S}\right)^{2} \mathrm{P}_{0.5}$ & - & $4 d^{9} 5 p^{2}$ & $\left({ }^{3} \mathrm{P}\right)^{2} \mathrm{P}_{15}$ & $57,184.0$ & $253,703.7$ & $4.60 \mathrm{E}+08$ & \# & $\mathrm{R}$ \\
\hline 20 & & $514.583(8)$ & 194,332 & 514.5798(11) & 0.003 & $4 d^{10} 5 \mathrm{~s}$ & $\left({ }^{1} \mathrm{~S}\right)^{2} \mathrm{~S}_{05}$ & - & $4 d^{10} 8 p$ & $\left({ }^{1} \mathrm{~S}^{2} \mathrm{P}_{15}\right.$ & 0.0 & $194,333.3$ & $3.30 \mathrm{E}+09$ & & B \\
\hline 750 & & $515.532(6)$ & $193,974.4$ & $515.5346(12)$ & -0.003 & $4 d^{10} 5 p$ & $\left({ }^{1}\right)^{2} \mathrm{P}_{05}$ & - & $4 d^{9} 5 p^{2}$ & $\left({ }^{3} \mathrm{P}\right)^{2} \mathrm{P}_{0.5}$ & $57,184.0$ & $251,157.4$ & $3.94 \mathrm{E}+09$ & & $\mathrm{R}$ \\
\hline 45 & & $519.544(6)$ & $192,476.5$ & $519.5369(11)$ & 0.007 & $4 d^{10} 5 p$ & $\left({ }^{1}\right)^{2} \mathrm{P}_{1.5}$ & - & $4 d^{9} 5 p^{2}$ & $\left({ }^{3} \mathrm{P}\right)^{4} \mathrm{P}_{0.5}$ & $61,527.0$ & $254,006.1$ & $2.39 \mathrm{E}+08$ & & TW \\
\hline 620 & & $520.357(6)$ & $192,175.8$ & $520.3544(15)$ & 0.003 & $4 d^{10} 5 p$ & $\left({ }^{1}\right)^{2} \mathrm{P}_{1.5}$ & - & $4 d^{9} 5 p^{2}$ & $\left({ }^{3} \mathrm{P}\right)^{2} \mathrm{P}_{1.5}$ & $61,527.0$ & $253,703.7$ & $8.31 \mathrm{E}+09$ & & $\mathrm{R}$ \\
\hline 120 & & $522.166(6)$ & $191,510.0$ & 522.1684(11) & -0.002 & $4 d^{10} 5 \mathrm{~s}$ & $\left({ }^{1} \mathrm{~S}^{2} \mathrm{~S}_{0.5}\right.$ & - & $4 d^{9} 5 s 5 p$ & $\left({ }^{3} \mathrm{D}\right)^{2} \mathrm{P}_{1.5}$ & 0.0 & $191,509.1$ & $1.07 \mathrm{E}+10$ & & K \\
\hline 300 & & $525.300(6)$ & $190,367.4$ & $525.2995(14)$ & 0.001 & $4 d^{10} 5 p$ & $\left({ }^{1} \mathrm{~S}\right)^{2} \mathrm{P}_{05}$ & - & $4 d^{9} 5 p^{2}$ & $\left({ }^{1} \mathrm{D}\right)^{2} \mathrm{D}_{1.5}$ & $57,184.0$ & $247,551.6$ & $1.85 \mathrm{E}+10$ & & $\mathrm{R}$ \\
\hline 250 & & $525.482(6)$ & $190,301.5$ & $525.4786(12)$ & 0.003 & $4 d^{10} 5 p$ & $\left({ }^{1} \mathrm{~S}\right)^{2} \mathrm{P}_{1.5}$ & - & $4 d^{9} 5 p^{2}$ & $\left({ }^{1} \mathrm{D}\right)^{2} \mathrm{~F}_{2.5}$ & $61,527.0$ & $251,829.7$ & $5.20 \mathrm{E}+09$ & & $\mathrm{R}$ \\
\hline
\end{tabular}


Table 3. Cont

\begin{tabular}{|c|c|c|c|c|c|c|c|c|c|c|c|c|c|c|c|}
\hline$I_{\mathrm{obs}}{ }^{\mathrm{a}}$ & $\mathrm{ch}^{\mathrm{b}}$ & $\lambda_{\text {obs }}{ }^{\mathrm{c}} \AA$ & $\sigma_{\text {obs }} \mathrm{cm}^{-1}$ & $\lambda_{\text {Ritz }}{ }^{\mathrm{d}} \AA$ & $\Delta \lambda_{\text {O-Ritz }}{ }^{\mathrm{e}} \AA$ & & Classification $^{\mathrm{f}}$ & & & & $E_{\text {low }} \mathrm{cm}^{-1}$ & $E_{\text {upp }} \mathrm{cm}^{-1}$ & $g A^{\mathrm{h}} \mathrm{S}^{-1}$ & & Lin. $\operatorname{Ref}^{\mathrm{i}}$ \\
\hline 540 & & $527.348(6)$ & $189,628.1$ & $527.3416(12)$ & 0.006 & $4 d^{10} 5 p$ & $\left({ }^{1} \mathrm{~S}\right)^{2} \mathrm{P}_{1.5}$ & - & $4 d^{9} 5 p^{2}$ & $\left({ }^{3} \mathrm{P}\right)^{2} \mathrm{P}_{0.5}$ & $61,527.0$ & $251,157.4$ & $3.46 \mathrm{E}+09$ & & $\mathrm{R}$ \\
\hline 230 & & $528.287(6)$ & $189,291.0$ & $528.2874(12)$ & 0.000 & $4 d^{10} 5 p$ & $\left({ }^{1} \mathrm{~S}\right)^{2} \mathrm{P}_{0.5}$ & - & $4 d^{9} 5 p^{2}$ & $\left({ }^{3} \mathrm{P}\right)^{4} \mathrm{~F}_{1.5}$ & $57,184.0$ & $246,474.9$ & $1.15 \mathrm{E}+06$ & \# & $\mathrm{R}$ \\
\hline 630 & & $529.200(6)$ & $188,964.5$ & $529.2002(19)$ & 0.000 & $4 d^{10} 5 p$ & $\left({ }^{1} \mathrm{~S}\right)^{2} \mathrm{P}_{1.5}$ & - & $4 d^{9} 5 p^{2}$ & $\left({ }^{3} \mathrm{P}\right)^{4} \mathrm{P}_{1.5}$ & $61,527.0$ & $250,491.4$ & 7.53E+09 & & $\mathrm{R}$ \\
\hline 450 & & $530.000(6)$ & $188,679.2$ & $530.0102(20)$ & -0.010 & $4 d^{10} 5 p$ & $\left({ }^{1} \mathrm{~S}\right)^{2} \mathrm{P}_{1.5}$ & - & $4 d^{9} 5 p^{2}$ & $\left({ }^{3} \mathrm{P}\right)^{2} \mathrm{D}_{2.5}$ & $61,527.0$ & $250,202.6$ & $2.73 \mathrm{E}+10$ & & $\mathrm{R}$ \\
\hline 460 & & $530.448(6)$ & $188,519.9$ & $530.4469(14)$ & 0.001 & $4 d^{10} 5 p$ & $\left({ }^{1} \mathrm{~S}\right)^{2} \mathrm{P}_{0.5}$ & - & $4 d^{9} 5 p^{2}$ & $\left({ }^{3} \mathrm{P}\right)^{2} \mathrm{D}_{1.5}$ & $57,184.0$ & $245,704.3$ & $4.21 \mathrm{E}+09$ & & $\mathrm{R}$ \\
\hline 300 & & $540.613(6)$ & $184,975.2$ & $540.6101(10)$ & 0.003 & $4 d^{10} 5 p$ & $\left({ }^{1} \mathrm{~S}\right)^{2} \mathrm{P}_{15}$ & - & $4 d^{9} 5 p^{2}$ & $\left({ }^{3} \mathrm{P}\right)^{4} \mathrm{P}_{25}$ & $61,527.0$ & $246,503.2$ & $4.37 \mathrm{E}+09$ & & $\mathrm{R}$ \\
\hline 200 & & $540.678(6)$ & $184,953.0$ & $540.6928(11)$ & -0.015 & $4 d^{10} 5 p$ & $\left({ }^{(} \mathrm{S}\right)^{2} \mathrm{P}_{1.5}$ & - & $4 d^{9} 5 p^{2}$ & $\left({ }^{3} \mathrm{P}\right)^{4} \mathrm{~F}_{1.5}$ & $61,527.0$ & $246,474.9$ & $6.21 \mathrm{E}+08$ & & $\mathrm{R}$ \\
\hline 480 & & $549.764(6)$ & $181,896.2$ & $549.764(6)$ & 0.000 & $4 d^{10} 5 p$ & $\left({ }^{1} \mathrm{~S}\right)^{2} \mathrm{P}_{1.5}$ & - & $4 d^{9} 5 p^{2}$ & $\left({ }^{1} \mathrm{D}\right){ }^{2} \mathrm{P}_{0.5}$ & $61,527.0$ & $243,423.3$ & $2.42 \mathrm{E}+09$ & & $\mathrm{R}$ \\
\hline 570 & & $550.518(6)$ & $181,647.1$ & $550.5186(13)$ & -0.001 & $4 d^{10} 5 p$ & $\left({ }^{1} \mathrm{~S}\right)^{2} \mathrm{P}_{0.5}$ & - & $4 d^{9} 5 p^{2}$ & $\left({ }^{3} \mathrm{P}\right)^{4} \mathrm{D}_{1.5}$ & $57,184.0$ & $238,830.9$ & $3.66 \mathrm{E}+09$ & & $\mathrm{R}$ \\
\hline 25 & & $552.660(6)$ & $180,943.1$ & $552.6573(11)$ & 0.003 & $4 d^{10} 5 s$ & $\left({ }^{1} \mathrm{~S}\right)^{2} \mathrm{~S}_{0.5}$ & - & $4 d^{9} 5 s 5 p$ & $\left({ }^{3} \mathrm{D}\right)^{4} \mathrm{D}_{1.5}$ & 0.0 & $180,943.95$ & $4.73 \mathrm{E}+08$ & & $\mathrm{~K}$ \\
\hline 15 & & $555.069(6)$ & $180,157.8$ & $555.0720(24)$ & -0.003 & $4 d^{10} 5 p$ & $\left({ }^{1} \mathrm{~S}\right)^{2} \mathrm{P}_{15}$ & - & $4 d^{9} 5 p^{2}$ & $\left({ }^{3} \mathrm{P}\right)^{4} \mathrm{D}_{0.5}$ & $61,527.0$ & $241,683.8$ & $3.56 \mathrm{E}+08$ & & TW \\
\hline 20 & & $555.501(6)$ & $180,017.7$ & $555.501(6)$ & 0.000 & $4 d^{10} 5 p$ & $\left({ }^{1} \mathrm{~S}\right)^{2} \mathrm{P}_{0.5}$ & - & $4 d^{9} 5 p^{2}$ & $\left({ }^{1} \mathrm{D}\right)^{2} \mathrm{~S}_{0.5}$ & $57,184.0$ & $237,201.7$ & $4.56 \mathrm{E}+09$ & & TW \\
\hline 390 & & $555.669(6)$ & $179,963.3$ & $555.674(4)$ & -0.005 & $4 d^{10} 5 p$ & $\left({ }^{1} \mathrm{~S}\right)^{2} \mathrm{P}_{0.5}$ & - & $4 d^{9} 5 p^{2}$ & $\left({ }^{1} \mathrm{D}\right){ }^{2} \mathrm{P}_{1.5}$ & $57,184.0$ & $237,145.6$ & $1.08 \mathrm{E}+09$ & & $\mathrm{R}$ \\
\hline 150 & & $557.662(6)$ & $179,320.1$ & $557.6595(13)$ & 0.003 & $4 d^{10} 5 s$ & $\left({ }^{1} \mathrm{~S}\right)^{2} \mathrm{~S}_{0.5}$ & - & $4 d^{9} 5 s 5 p$ & $\left({ }^{1} \mathrm{D}\right)^{2} \mathrm{P}_{0.5}$ & 0.0 & $179,320.9$ & $1.47 \mathrm{E}+09$ & & K \\
\hline 150 & & $559.857(6)$ & $178,617.0$ & $559.8576(11)$ & -0.001 & $4 d^{10} 5 s$ & $\left({ }^{1} \mathrm{~S}\right)^{2} \mathrm{~S}_{0.5}$ & - & $4 d^{9} 5 \mathrm{~s} 5 \mathrm{p}$ & $\left({ }^{1} \mathrm{D}\right)^{2} \mathrm{P}_{1.5}$ & 0.0 & $178,616.85$ & $1.27 \mathrm{E}+10$ & & K \\
\hline 130 & & $561.210(6)$ & $178,186.4$ & $561.2059(11)$ & 0.004 & $4 d^{10} 5 s$ & $\left({ }^{1} \mathrm{~S}\right)^{2} \mathrm{~S}_{0.5}$ & - & $4 d^{9} 5 s 5 p$ & $\left({ }^{3} \mathrm{D}\right)^{4} \mathrm{D}_{0.5}$ & 0.0 & $178,187.72$ & $5.47 \mathrm{E}+09$ & & K \\
\hline 200 & & $562.214(6)$ & $177,868.2$ & $562.2155(11)$ & -0.001 & $4 d^{10} 5 s$ & $\left({ }^{1} \mathrm{~S}\right)^{2} \mathrm{~S}_{0.5}$ & - & $4^{10} 7 \mathrm{p}$ & $\left({ }^{1} \mathrm{~S}\right)^{2} \mathrm{P}_{1.5}$ & 0.0 & $177,867.74$ & $5.56 \mathrm{E}+09$ & & B, TW \\
\hline 160 & & $564.131(6)$ & $177,263.8$ & $564.1323(11)$ & -0.001 & $4 d^{10} 5 s$ & $\left({ }^{1} \mathrm{~S}\right)^{2} \mathrm{~S}_{0.5}$ & - & $4^{10} 7 \mathrm{p}$ & $\left({ }^{1} \mathrm{~S}\right)^{2} \mathrm{P}_{0.5}$ & 0.0 & $177,263.38$ & 3.77E+09 & & B, TW \\
\hline 480 & & $569.421(6)$ & $175,617.0$ & $569.416(4)$ & 0.005 & $4 d^{10} 5 p$ & $\left({ }^{1} \mathrm{~S}\right)^{2} \mathrm{P}_{1.5}$ & - & $4 d^{9} 5 p^{2}$ & $\left({ }^{1} \mathrm{D}\right)^{2} \mathrm{P}_{1.5}$ & $61,527.0$ & $237,145.6$ & $1.72 \mathrm{E}+09$ & & $\mathrm{R}$ \\
\hline 80 & & $569.677(6)$ & $175,538.1$ & $569.6728(12)$ & 0.004 & $4 d^{10} 5 s$ & $\left({ }^{1} \mathrm{~S}\right)^{2} \mathrm{~S}_{0.5}$ & - & $4 d^{9} 5 s 5 p$ & $\left({ }^{1} \mathrm{D}\right)^{2} \mathrm{D}_{1.5}$ & 0.0 & $175,539.35$ & $7.80 \mathrm{E}+08$ & & $\mathrm{~K}$ \\
\hline 80 & & $583.723(6)$ & $171,314.1$ & $583.718(3)$ & 0.005 & $4 d^{10} 5 s$ & $\left({ }^{1} \mathrm{~S}\right)^{2} \mathrm{~S}_{0.5}$ & - & $4 d^{9} 5 s 5 p$ & $\left({ }^{3} \mathrm{D}\right){ }^{4} \mathrm{P}_{0.5}$ & 0.0 & $171,315.7$ & $9.39 \mathrm{E}+08$ & & TW \\
\hline 50 & & $585.440(6)$ & $170,811.7$ & $585.4331(18)$ & 0.007 & $4 d^{10} 5 s$ & $\left({ }^{1} \mathrm{~S}\right)^{2} \mathrm{~S}_{0.5}$ & - & $4 d^{9} 5 s 5 p$ & $\left({ }^{3} \mathrm{D}\right){ }^{4} \mathrm{~F}_{1.5}$ & 0.0 & $170,813.7$ & $2.95 \mathrm{E}+07$ & & K \\
\hline 100 & & $597.596(6)$ & $167,337.1$ & $597.5885(13)$ & 0.008 & $4 d^{10} 5 s$ & $\left({ }^{1} \mathrm{~S}\right)^{2} \mathrm{~S}_{0.5}$ & - & $4 d^{9} 5 \mathrm{~s} 5 \mathrm{p}$ & $\left({ }^{3} \mathrm{D}\right){ }^{4} \mathrm{P}_{1.5}$ & 0.0 & $167,339.24$ & $1.18 \mathrm{E}+09$ & & B, TW \\
\hline 30 & & $635.672(8)$ & $157,313.8$ & $635.673(6)$ & -0.001 & $4 d^{10} 5 p$ & $\left({ }^{1} \mathrm{~S}\right)^{2} \mathrm{P}_{0.5}$ & - & $4 d^{10} 12 s$ & $\left({ }^{1} S\right)^{2} S_{0.5}$ & $57,184.0$ & $214,497.7$ & $3.71 \mathrm{E}+07$ & & B \\
\hline 32 & & $648.185(8)$ & $154,276.9$ & 648.1801(15) & 0.005 & $4 d^{10} 5 p$ & $\left({ }^{1} \mathrm{~S}\right)^{2} \mathrm{P}_{0.5}$ & - & $4 d^{10} 11 \mathrm{~s}$ & $\left({ }^{1} \mathrm{~S}\right)^{2} \mathrm{~S}_{0.5}$ & $57,184.0$ & $211,462.1$ & $5.21 \mathrm{E}+07$ & & B \\
\hline 33 & & 653.721(8) & $152,970.5$ & $653.720(6)$ & 0.001 & $4 d^{10} 5 p$ & $\left({ }^{1} \mathrm{~S}\right)^{2} \mathrm{P}_{1.5}$ & - & $4 d^{10} 12 s$ & $\left({ }^{1} \mathrm{~S}\right)^{2} \mathrm{~S}_{0.5}$ & $61,527.0$ & $214,497.7$ & $6.79 \mathrm{E}+07$ & & B \\
\hline 41 & & $665.979(8)$ & $150,154.9$ & $665.9794(17)$ & 0.000 & $4 d^{10} 5 p$ & $\left({ }^{1} \mathrm{~S}\right)^{2} \mathrm{P}_{0.5}$ & - & $4 d^{10} 9 \mathrm{~d}$ & $\left({ }^{1} \mathrm{~S}\right)^{2} \mathrm{D}_{1.5}$ & $57,184.0$ & $207,338.8$ & $9.52 \mathrm{E}+07$ & & B \\
\hline 40 & & $666.963(8)$ & $149,933.4$ & $666.9552(15)$ & 0.008 & $4 d^{10} 5 p$ & $\left({ }^{1} \mathrm{~S}\right)^{2} \mathrm{P}_{1.5}$ & - & $4 d^{10} 11 s$ & $\left({ }^{1} \mathrm{~S}\right)^{2} \mathrm{~S}_{0.5}$ & $61,527.0$ & $211,462.1$ & $9.55 \mathrm{E}+07$ & & B \\
\hline 40 & & 667.177(8) & $149,885.3$ & $667.1807(15)$ & -0.004 & $4 d^{10} 5 p$ & $\left({ }^{1} \mathrm{~S}\right)^{2} \mathrm{P}_{0.5}$ & - & $4 \mathrm{~d}^{10} 10 \mathrm{~s}$ & $\left({ }^{1} \mathrm{~S}\right)^{2} \mathrm{~S}_{0.5}$ & $57,184.0$ & $207,068.43$ & $7.66 \mathrm{E}+07$ & & B \\
\hline 80 & & $685.273(6)$ & $145,927.2$ & 685.2779(16) & -0.005 & $4 d^{10} 5 s$ & $\left({ }^{1} \mathrm{~S}\right)^{2} \mathrm{~S}_{0.5}$ & - & $4 d^{10} 6 p$ & $\left.{ }^{(1} \mathrm{S}\right)^{2} \mathrm{P}_{1.5}$ & 0.0 & $145,926.21$ & $4.63 \mathrm{E}+07$ & & B, TW \\
\hline 41 & & 685.612(8) & $145,855.1$ & $685.6232(17)$ & -0.011 & $4 d^{10} 5 p$ & $\left({ }^{1} \mathrm{~S}\right)^{2} \mathrm{P}_{1.5}$ & - & $4 d^{10} 9 d$ & $\left({ }^{1} \mathrm{~S}\right)^{2} \mathrm{D}_{2.5}$ & $61,527.0$ & $207,379.7$ & $1.68 \mathrm{E}+08$ & & B \\
\hline 67 & & $685.815(8)$ & $145,811.9$ & $685.8156(17)$ & -0.001 & $4 d^{10} 5 p$ & $\left({ }^{1} \mathrm{~S}\right)^{2} \mathrm{P}_{1.5}$ & - & $4 d^{10} 9 d$ & $\left({ }^{1} \mathrm{~S}\right)^{2} \mathrm{D}_{1.5}$ & $61,527.0$ & $207,338.8$ & $1.88 \mathrm{E}+07$ & & B \\
\hline 48 & & $687.076(8)$ & $145,544.3$ & $687.0896(15)$ & -0.014 & $4 d^{10} 5 p$ & $\left({ }^{1} \mathrm{~S}\right)^{2} \mathrm{P}_{1.5}$ & - & $4 \mathrm{~d}^{10} 10 \mathrm{~s}$ & $\left({ }^{1} \mathrm{~S}\right)^{2} \mathrm{~S}_{0.5}$ & $61,527.0$ & $207,068.43$ & $1.41 \mathrm{E}+08$ & & B \\
\hline 200 & & $691.610(6)$ & $144,590.2$ & $691.6140(17)$ & -0.004 & $4 d^{10} 5 s$ & $\left({ }^{1} \mathrm{~S}\right)^{2} \mathrm{~S}_{0.5}$ & - & $4 d^{10} 6 p$ & $\left({ }^{1} \mathrm{~S}\right)^{2} \mathrm{P}_{0.5}$ & 0.0 & $144,589.32$ & $2.10 \mathrm{E}+07$ & & B, TW \\
\hline 50 & & $696.399(8)$ & $143,595.8$ & 696.4064(13) & -0.007 & $4 d^{10} 5 p$ & $\left({ }^{1} \mathrm{~S}\right)^{2} \mathrm{P}_{0.5}$ & - & $4 d^{10} 8 d$ & $\left({ }^{1} \mathrm{~S}\right)^{2} \mathrm{D}_{1.5}$ & $57,184.0$ & $200,778.32$ & $1.79 \mathrm{E}+08$ & & B \\
\hline 65 & & 698.422(8) & $143,179.9$ & $698.4276(15)$ & -0.006 & $4 d^{10} 5 p$ & $\left({ }^{1} \mathrm{~S}\right)^{2} \mathrm{P}_{0.5}$ & - & $4 d^{10} 9 s$ & $\left({ }^{1} \mathrm{~S}\right)^{2} \mathrm{~S}_{0.5}$ & $57,184.0$ & $200,362.77$ & $1.20 \mathrm{E}+08$ & & B \\
\hline 68 & & $717.834(8)$ & $139,308.0$ & $717.8287(12)$ & 0.005 & $4 d^{10} 5 p$ & $\left({ }^{1} \mathrm{~S}\right)^{2} \mathrm{P}_{1.5}$ & - & $4 d^{10} 8 \mathrm{~d}$ & $\left({ }^{1} \mathrm{~S}\right)^{2} \mathrm{D}_{2.5}$ & $61,527.0$ & $200,836.01$ & $3.09 \mathrm{E}+08$ & & B \\
\hline 60 & & $718.135(8)$ & $139,249.6$ & $718.1260(12)$ & 0.009 & $4 d^{10} 5 p$ & $\left({ }^{1} \mathrm{~S}\right)^{2} \mathrm{P}_{1.5}$ & - & $4 d^{10} 8 d$ & $\left({ }^{1} S^{2} D_{1.5}\right.$ & $61,527.0$ & $200,778.32$ & $3.45 \mathrm{E}+07$ & & B \\
\hline
\end{tabular}


Table 3. Cont

\begin{tabular}{|c|c|c|c|c|c|c|c|c|c|c|c|c|c|c|c|}
\hline$I_{\mathrm{obs}}{ }^{\mathrm{a}}$ & $\mathrm{ch}^{\mathrm{b}}$ & $\lambda_{\text {obs }}{ }^{\mathrm{c}} \AA$ & $\sigma_{\mathrm{obs}} \mathrm{cm}^{-1}$ & $\lambda_{\text {Ritz }}{ }^{\mathrm{d}} \AA$ & $\Delta \lambda_{\text {O-Ritz }}{ }^{\mathrm{e}} \AA$ & & Classification $^{\mathrm{f}}$ & & & & $E_{\text {low }} \mathrm{cm}^{-1}$ & $E_{\text {upp }} \mathrm{cm}^{-1}$ & $g A^{\mathrm{h}} \mathrm{S}^{-1}$ & & Lin. $\operatorname{Ref}^{\mathrm{i}}$ \\
\hline 70 & & $720.281(8)$ & $138,834.7$ & $720.2755(15)$ & 0.006 & $4 d^{10} 5 p$ & $\left({ }^{1} \mathrm{~S}\right)^{2} \mathrm{P}_{1.5}$ & - & $4 d^{10} 9 \mathrm{~s}$ & $\left({ }^{1} \mathrm{~S}\right)^{2} \mathrm{~S}_{0.5}$ & $61,527.0$ & $200,362.77$ & $2.20 \mathrm{E}+08$ & & B \\
\hline 15 & & $752.699(6)$ & $132,855.2$ & 752.7014(21) & -0.002 & $4 d^{10} 5 p$ & $\left({ }^{1} \mathrm{~S}\right)^{2} \mathrm{P}_{0.5}$ & - & $4 d^{10} 7 d$ & $\left({ }^{1} \mathrm{~S}\right)^{2} \mathrm{D}_{1.5}$ & $57,184.0$ & $190,038.8$ & $3.91 \mathrm{E}+08$ & & B, TW \\
\hline 20 & & $756.484(6)$ & $132,190.5$ & $756.4840(21)$ & 0.000 & $4 d^{10} 5 p$ & $\left({ }^{1} \mathrm{~S}\right)^{2} \mathrm{P}_{0.5}$ & - & $4 d^{10} 8 s$ & $\left({ }^{1} \mathrm{~S}\right)^{2} \mathrm{~S}_{0.5}$ & $57,184.0$ & $189,374.5$ & $2.04 \mathrm{E}+08$ & & B, TW \\
\hline 10 & & $777.547(6)$ & $128,609.6$ & $777.549(3)$ & -0.002 & $4 d^{10} 5 p$ & $\left({ }^{1} \mathrm{~S}\right)^{2} \mathrm{P}_{1.5}$ & - & $4 d^{10} 7 \mathrm{~d}$ & $\left({ }^{1} \mathrm{~S}\right)^{2} \mathrm{D}_{2.5}$ & $61,527.0$ & $190,136.3$ & $6.60 \mathrm{E}+08$ & & B, TW \\
\hline 65 & & $778.142(8)$ & $128,511.2$ & $778.1387(21)$ & 0.003 & $4 d^{10} 5 p$ & $\left({ }^{1} \mathrm{~S}\right)^{2} \mathrm{P}_{1.5}$ & - & $4 d^{10} 7 \mathrm{~d}$ & $\left({ }^{1} \mathrm{~S}\right)^{2} \mathrm{D}_{1.5}$ & $61,527.0$ & $190,038.8$ & $7.34 \mathrm{E}+07$ & & B \\
\hline 30 & & $782.187(6)$ & $127,846.7$ & $782.1819(20)$ & 0.005 & $4 d^{10} 5 p$ & $\left({ }^{1} \mathrm{~S}\right)^{2} \mathrm{P}_{1.5}$ & - & $4 d^{10} 8 \mathrm{~s}$ & $\left({ }^{1} \mathrm{~S}\right)^{2} \mathrm{~S}_{0.5}$ & $61,527.0$ & $189,374.5$ & $3.73 \mathrm{E}+08$ & & B, TW \\
\hline 180 & & $882.207(6)$ & $113,352.1$ & $882.2095(22)$ & -0.003 & $4 d^{10} 5 p$ & $\left({ }^{1} \mathrm{~S}\right)^{2} \mathrm{P}_{0.5}$ & - & $4 d^{10} 6 \mathrm{~d}$ & $\left({ }^{1} \mathrm{~S}\right)^{2} \mathrm{D}_{1.5}$ & $57,184.0$ & $170,535.76$ & $1.12 \mathrm{E}+09$ & & B, TW \\
\hline 150 & & $890.870(6)$ & $112,249.8$ & $890.8639(23)$ & 0.006 & $4 d^{10} 5 p$ & $\left({ }^{1} \mathrm{~S}\right)^{2} \mathrm{P}_{0.5}$ & - & $4 \mathrm{~d}^{10} 7 \mathrm{~s}$ & $\left({ }^{1} \mathrm{~S}\right)^{2} \mathrm{~S}_{0.5}$ & $57,184.0$ & $169,434.59$ & $4.00 \mathrm{E}+08$ & & B, TW \\
\hline 120 & & $915.824(6)$ & $109,191.3$ & $915.8196(21)$ & 0.004 & $4 d^{10} 5 p$ & $\left({ }^{1} \mathrm{~S}\right)^{2} \mathrm{P}_{1.5}$ & - & $4 d^{10} 6 \mathrm{~d}$ & $\left({ }^{1} \mathrm{~S}\right)^{2} \mathrm{D}_{2.5}$ & $61,527.0$ & $170,718.81$ & $1.84 \mathrm{E}+09$ & & B, TW \\
\hline 25 & & $917.355(6)$ & $109,009.1$ & 917.3575(20) & -0.002 & $4 d^{10} 5 p$ & $\left({ }^{1} \mathrm{~S}\right)^{2} \mathrm{P}_{1.5}$ & - & $4 d^{10} 6 \mathrm{~d}$ & $\left({ }^{1} \mathrm{~S}\right)^{2} \mathrm{D}_{1.5}$ & $61,527.0$ & $170,535.76$ & $2.04 \mathrm{E}+08$ & & B, TW \\
\hline 120 & & $926.723(8)$ & $107,907.1$ & $926.7189(21)$ & 0.004 & $4 d^{10} 5 p$ & $\left({ }^{1} \mathrm{~S}\right)^{2} \mathrm{P}_{1.5}$ & - & $4 d^{10} 7 s$ & $\left({ }^{1} \mathrm{~S}\right)^{2} \mathrm{~S}_{0.5}$ & $61,527.0$ & $169,434.59$ & $7.20 \mathrm{E}+08$ & & B \\
\hline 200 & & $1153.839(8)$ & $86,667.2$ & $1153.844(5)$ & -0.005 & $d^{9} 5 s 5 p$ & $\left({ }^{3} \mathrm{D}\right){ }^{4} \mathrm{P}_{1.5}$ & - & $4 d^{9} 5 p^{2}$ & $\left({ }^{3} \mathrm{P}\right)^{4} \mathrm{P}_{0.5}$ & $167,339.24$ & $254,006.1$ & $3.60 \mathrm{E}+08$ & \# & TW \\
\hline 6 & & $1162.895(8)$ & $85,992.3$ & $1162.903(6)$ & -0.008 & $d^{9} 5 s 5 p$ & $\left({ }^{3} \mathrm{D}\right){ }^{4} \mathrm{~F}_{2.5}$ & - & $4 d^{9} 5 p^{2}$ & $\left({ }^{3} \mathrm{P}\right)^{2} \mathrm{~F}_{3.5}$ & $167,465.9$ & $253,457.6$ & $3.59 \mathrm{E}+09$ & & TW \\
\hline 50 & & 1201.523(8) & $83,227.7$ & 1201.532(5) & -0.009 & $4 d^{9} 5 s^{2}$ & $\left({ }^{2} \mathrm{D}\right)^{2} \mathrm{D}_{2.5}$ & - & $4 d^{9} 5 \mathrm{~s} 5 \mathrm{p}$ & $\left({ }^{3} \mathrm{D}\right)^{2} \mathrm{~F}_{2.5}$ & $115,572.19$ & $198,799.3$ & $2.25 \mathrm{E}+09$ & & TW \\
\hline 80 & & $1210.468(8)$ & $826,12.7$ & $1210.465(5)$ & 0.003 & $d^{9} 5 s 5 p$ & $\left({ }^{3} \mathrm{D}\right)^{4} \mathrm{P}_{2.5}$ & - & $4 d^{9} 5 p^{2}$ & $\left({ }^{3} \mathrm{P}\right)^{4} \mathrm{P}_{2.5}$ & $163,890.3$ & $246,503.2$ & $2.49 \mathrm{E}+09$ & & TW \\
\hline 300 & $\mathrm{w}$ & $1254.458(16)$ & $79,715.7$ & $1254.465(7)$ & -0.007 & $4 d^{9} 5 s^{2}$ & $\left({ }^{2} \mathrm{D}\right)^{2} \mathrm{D}_{1.5}$ & - & $4 d^{9} 5 \mathrm{~s} 5 \mathrm{p}$ & $\left({ }^{3} D\right)^{2} D_{1.5}$ & $122,419.73$ & $202,135.0$ & $7.59 \mathrm{E}+09$ & & B, TW \\
\hline 300 & $\mathrm{w}$ & $1260.567(16)$ & $79,329.4$ & $1260.551(6)$ & 0.016 & $4 d^{9} 5 s^{2}$ & $\left({ }^{2} \mathrm{D}\right)^{2} \mathrm{D}_{2.5}$ & - & $4 d^{9} 5 s 5 p$ & $\left({ }^{3} \mathrm{D}\right)^{2} \mathrm{D}_{2.5}$ & $115,572.19$ & $194,902.6$ & $1.49 \mathrm{E}+10$ & & TW \\
\hline 200 & & $(1263.152)$ & $79,167.0$ & 1263.201(5) & & $4 d^{9} 5 s 5 p$ & $\left({ }^{3} \mathrm{D}\right){ }^{4} \mathrm{P}_{1.5}$ & - & $4 d^{9} 5 p^{\frac{2}{2}}$ & $\left({ }^{3} \mathrm{P}\right){ }^{4} \mathrm{P}_{2.5}$ & $167,339.24$ & $246,503.2$ & $3.92 \mathrm{E}+09$ & & TW \\
\hline 100 & & (1263.594) & $79,139.3$ & $1263.653(6)$ & & $4 d^{9} 5 s 5 p$ & $\left({ }^{3} \mathrm{D}\right){ }^{4} \mathrm{P}_{1.5}$ & - & $4 d^{9} 5 p^{2}$ & $\left({ }^{3} \mathrm{P}\right)^{4} \mathrm{~F}_{1.5}$ & $167,339.24$ & $246,474.9$ & $3.06 \mathrm{E}+08$ & & TW \\
\hline 15 & & $1285.588(8)$ & $77,785.4$ & $1285.577(6)$ & 0.011 & $4 d^{9} 5 s 5 p$ & $\left({ }^{3} \mathrm{D}\right)^{4} \mathrm{D}_{3.5}$ & - & $4 d^{9} 5 p^{2}$ & $\left({ }^{1} \mathrm{D}\right)^{2} \mathrm{~F}_{2.5}$ & $174,043.59$ & $251,829.7$ & $4.50 \mathrm{E}+06$ & \# & TW \\
\hline 20 & & 1287.752(8) & $77,654.7$ & 1287.762(5) & -0.010 & $4 d^{9} 5 s 5 p$ & $\left({ }^{3} D\right){ }^{4} P_{2.5}$ & - & $4 d^{9} 5 p^{2}$ & $\left({ }^{3} \mathrm{P}\right)^{2} \mathrm{~F}_{2.5}$ & $163,890.3$ & $241,544.42$ & $1.29 \mathrm{E}+09$ & & TW \\
\hline 150 & & $1294.468(8)$ & $77,251.8$ & $1294.468(5)$ & 0.000 & $4 d^{9} 5 s 5 p$ & $\left({ }^{3} \mathrm{D}\right){ }^{4} \mathrm{~F}_{3.5}$ & - & $4 d^{9} 5 p^{2}$ & $\left({ }^{3} \mathrm{P}\right)^{4} \mathrm{~F}_{4.5}$ & $167,308.1$ & $244,559.9$ & $5.06 \mathrm{E}+09$ & & TW \\
\hline 250 & $\mathrm{w}$ & $1309.269(16)$ & $76,378.5$ & $1309.251(6)$ & 0.018 & $4 d^{9} 5 s^{2}$ & $\left({ }^{2} \mathrm{D}\right)^{2} \mathrm{D}_{1.5}$ & - & $4 d^{9} 5 s 5 p$ & $\left({ }^{3} \mathrm{D}\right)^{2} \mathrm{~F}_{2.5}$ & $122,419.73$ & $198,799.3$ & 8.07E+09 & & TW \\
\hline 15 & & $1315.880(8)$ & $75,994.8$ & $1315.880(8)$ & 0.000 & $4 d^{9} 5 s 5 p$ & $\left({ }^{3} \mathrm{D}\right)^{4} \mathrm{D}_{2.5}$ & - & $4 d^{9} 5 p^{2}$ & $\left({ }^{3} \mathrm{P}\right)^{4} \mathrm{P}_{1.5}$ & $174,496.6$ & $250,491.4$ & $1.81 \mathrm{E}+09$ & & TW \\
\hline 280 & $\mathrm{w}$ & 1316.430(16) & $75,963.0$ & $1316.440(7)$ & -0.010 & $4 d^{9} 5 s^{2}$ & $\left({ }^{2} \mathrm{D}\right)^{2} \mathrm{D}_{1.5}$ & - & $4 d^{9} 5 s 5 p$ & $\left({ }^{3} \mathrm{D}\right)^{2} \mathrm{P}_{0.5}$ & $122,419.73$ & $198,382.2$ & $3.01 \mathrm{E}+09$ & & TW \\
\hline 70 & & 1318.399(8) & $75,849.6$ & $1318.409(5)$ & -0.010 & $4 d^{9} 5 s 5 p$ & $\left({ }^{3} \mathrm{D}\right){ }^{4} \mathrm{P}_{2.5}$ & - & $4 d^{9} 5 p^{2}$ & $\left({ }^{1} \mathrm{D}\right)^{2} \mathrm{D}_{2.5}$ & $163,890.3$ & $239,739.3$ & $1.16 \mathrm{E}+09$ & & TW \\
\hline 60 & & $1318.946(8)$ & $75,818.1$ & $1318.941(6)$ & 0.005 & $4 d^{9} 5 s 5 p$ & $\left.\left({ }^{3} \mathrm{D}\right)\right)^{4} \mathrm{D}_{0.5}$ & - & $4 d^{9} 5 p^{2}$ & $\left({ }^{3} \mathrm{P}\right)^{4} \mathrm{P}_{0.5}$ & $178,187.72$ & $254,006.1$ & $1.12 \mathrm{E}+08$ & \# & TW \\
\hline 100 & & $1320.314(8)$ & $75,739.6$ & $1320.315(6)$ & -0.001 & $4 d^{9} 5 s 5 p$ & $\left({ }^{1} \mathrm{D}\right)^{2} \mathrm{~F}_{2.5}$ & - & $4 d^{9} 5 p^{2}$ & $\left({ }^{1} \mathrm{D}\right)^{2} \mathrm{~F}_{2.5}$ & $176,090.19$ & $251,829.7$ & $3.21 \mathrm{E}+09$ & & TW \\
\hline 150 & & 1321.683(8) & $75,661.1$ & 1321.681(7) & 0.002 & $4 d^{9} 5 s 5 p$ & $\left({ }^{3} \mathrm{D}\right){ }^{4} \mathrm{~F}_{1.5}$ & - & $4 d^{9} 5 p^{2}$ & $\left({ }^{3} \mathrm{P}\right)^{4} \mathrm{~F}_{1.5}$ & $170,813.7$ & $246,474.9$ & $4.82 \mathrm{E}+09$ & & TW \\
\hline 200 & & $1322.526(8)$ & $75,612.9$ & $1322.536(6)$ & -0.010 & $4 d^{9} 5 \mathrm{~s} 5 \mathrm{p}$ & $\left({ }^{3} \mathrm{D}\right){ }^{4} \mathrm{~F}_{4.5}$ & - & $4 d^{9} 5 p^{2}$ & $\left({ }^{3} \mathrm{P}\right)^{4} \mathrm{~F}_{4.5}$ & $168,947.6$ & $244,559.9$ & $1.78 \mathrm{E}+10$ & & TW \\
\hline 250 & $\mathrm{w}$ & $1323.944(16)$ & $75,531.9$ & $1323.944(16)$ & 0.000 & $4 d^{9} 5 s^{2}$ & $\left({ }^{2} \mathrm{D}\right)^{2} \mathrm{D}_{2.5}$ & - & $4 d^{9} 5 s 5 p$ & $\left({ }^{3} \mathrm{D}\right)^{2} \mathrm{~F}_{3.5}$ & $115,572.19$ & $191,104.1$ & $1.75 \mathrm{E}+10$ & & TW \\
\hline 80 & & $1349.914(8)$ & $74,078.8$ & $1349.919(6)$ & -0.005 & $4 d^{9} 5 s 5 p$ & $\left({ }^{3} \mathrm{D}\right){ }^{4} \mathrm{~F}_{2.5}$ & - & $4 d^{9} 5 p^{\frac{2}{2}}$ & $\left({ }^{3} \mathrm{P}\right)^{2} \mathrm{~F}_{2.5}$ & $167,465.9$ & $241,544.42$ & 6.37E+09 & & TW \\
\hline 60 & & $1357.284(8)$ & $73,676.5$ & $1357.282(7)$ & 0.002 & $4 d^{10} 5 \mathrm{~d}$ & $\left({ }^{1} \mathrm{~S}\right)^{2} \mathrm{D}_{1.5}$ & - & $4 d^{9} 5 s 5 p$ & $\left({ }^{3} \mathrm{D}\right)^{2} \mathrm{D}_{1.5}$ & $128,458.36$ & $202,135.0$ & $4.03 \mathrm{E}+09$ & & TW \\
\hline 100 & & 1358.998(8) & $73,583.6$ & $1359.002(7)$ & -0.004 & $4 d^{9} 5 s 5 p$ & $\left({ }^{3} \mathrm{D}\right)^{4} \mathrm{~F}_{3.5}$ & - & $4 d^{9} 5 p^{2}$ & $\left({ }^{1} \mathrm{D}\right)^{2} \mathrm{~F}_{3.5}$ & $167,308.1$ & $240,891.5$ & $1.66 \mathrm{E}+09$ & & TW \\
\hline 200 & & $1362.445(8)$ & $73,397.5$ & $1362.448(8)$ & -0.003 & $4 d^{9} 5 s 5 p$ & $\left({ }^{1} \mathrm{D}\right)^{2} \mathrm{~F}_{3.5}$ & - & $4 d^{9} 5 p^{2}$ & $\left({ }^{3} \mathrm{P}\right)^{2} \mathrm{~F}_{3.5}$ & $180,060.3$ & $253,457.6$ & $1.10 \mathrm{E}+10$ & & TW \\
\hline 15 & & $1370.424(8)$ & $72,970.1$ & 1370.432(7) & -0.008 & $4 d^{9} 5 s 5 p$ & $\left({ }^{3} D\right)^{4} \mathrm{D}_{0.5}$ & - & $4 d^{9} 5 p^{2}$ & $\left({ }^{3} \mathrm{P}\right)^{2} \mathrm{P}_{0.5}$ & $178,187.72$ & $251,157.4$ & $6.79 \mathrm{E}+08$ & & TW \\
\hline 8 & $\mathrm{f}$ & $1378.569(16)$ & $72,539.0$ & 1378.539(8) & 0.030 & $4 d^{9} 5 s 5 p$ & $\left({ }^{1} \mathrm{D}\right){ }^{2} \mathrm{P}_{1.5}$ & - & $4 d^{9} 5 p^{2}$ & $\left({ }^{3} \mathrm{P}\right)^{2} \mathrm{P}_{0.5}$ & $178,616.85$ & $251,157.4$ & $4.19 \mathrm{E}+08$ & & TW \\
\hline 160 & & $1380.066(8)$ & $72,460.3$ & $1380.079(5)$ & -0.013 & $4 d^{9} 5 s 5 p$ & $\left({ }^{3} \mathrm{D}\right)^{4} \mathrm{D}_{3.5}$ & - & $4 d^{9} 5 p^{2}$ & $\left({ }^{3} \mathrm{P}\right)^{4} \mathrm{P}_{2.5}$ & $174,043.59$ & $246,503.2$ & $6.43 \mathrm{E}+09$ & & TW \\
\hline 160 & & $1380.638(8)$ & $72,430.3$ & $1380.621(6)$ & 0.017 & $4 d^{9} 5 s 5 p$ & $\left({ }^{3} \mathrm{D}\right)^{4} \mathrm{~F}_{3.5}$ & - & $4 d^{9} 5 p^{2}$ & $\left({ }^{1} \mathrm{D}\right)^{2} \mathrm{D}_{2.5}$ & $167,308.1$ & $239,739.3$ & $1.06 \mathrm{E}+09$ & & TW \\
\hline 180 & & $1383.510(8)$ & $72,279.9$ & $1383.510(6)$ & 0.000 & $4 d^{9} 5 s 5 p$ & $\left({ }^{3} D\right)^{4} \mathrm{P}_{2.5}$ & - & $4 d^{9} 5 p^{2}$ & $\left({ }^{3} \mathrm{P}\right)^{4} \mathrm{D}_{3.5}$ & $163,890.3$ & $236,170.2$ & $1.06 \mathrm{E}+10$ & & TW \\
\hline 40 & & $1389.976(8)$ & $71,943.7$ & $1389.972(7)$ & 0.004 & $4 d^{9} 5 s 5 p$ & $\left({ }^{3} \mathrm{D}\right){ }^{4} \mathrm{~F}_{4.5}$ & - & $4 d^{9} 5 p^{2}$ & $\left({ }^{1} \mathrm{D}\right){ }^{2} \mathrm{~F}_{3.5}$ & $168,947.6$ & $240,891.5$ & $4.36 \mathrm{E}+08$ & & TW \\
\hline 8 & $\mathrm{f}$ & $1390.554(16)$ & $71,913.8$ & $1390.558(5)$ & -0.004 & $4 d^{9} 5 s^{2}$ & $\left({ }^{2} D\right)^{2} \mathrm{D}_{1.5}$ & - & $4 d^{10} 8 p$ & $\left({ }^{1} \mathrm{~S}\right)^{2} \mathrm{P}_{1.5}$ & $122,419.73$ & $194,333.3$ & $1.89 \mathrm{E}+07$ & \# & TW \\
\hline 20 & & $1397.429(8)$ & $71,560.0$ & 1397.415(6) & 0.014 & $4 d^{9} 5 s 5 p$ & $\left({ }^{3} \mathrm{D}\right)^{4} \mathrm{P}_{2.5}$ & - & $4 d^{9} 5 p^{2}$ & $\left({ }^{3} \mathrm{P}\right)^{4} \mathrm{D}_{2.5}$ & $163,890.3$ & $235,451.0$ & $2.21 \mathrm{E}+09$ & & TW \\
\hline
\end{tabular}


Table 3. Cont

\begin{tabular}{|c|c|c|c|c|c|c|c|c|c|c|c|c|c|c|c|}
\hline$I_{\mathrm{obs}}{ }^{\mathrm{a}}$ & $\mathrm{ch}^{\mathrm{b}}$ & $\lambda_{\text {obs }}{ }^{\mathrm{c}} \AA$ & $\sigma_{\mathrm{obs}} \mathrm{cm}^{-1}$ & $\lambda_{\text {Ritz }}{ }^{\mathrm{d}} \AA$ & $\Delta \lambda_{\text {O-Ritz }}{ }^{\mathrm{e}} \AA$ & & Classification & & & & $E_{\text {low }} \mathrm{cm}^{-1}$ & $E_{\text {upp }} \mathrm{cm}^{-1}$ & $g A^{\mathrm{h}} \mathrm{S}^{-1}$ & & Lin. $\operatorname{Ref}^{\mathrm{i}}$ \\
\hline 200 & & $1398.755(8)$ & $71,492.1$ & $1398.765(7)$ & -0.010 & $4 d^{9} 5 s 5 p$ & $\left({ }^{3} \mathrm{D}\right)^{4} \mathrm{P}_{1.5}$ & - & $4 d^{9} 5 p^{2}$ & $\left({ }^{3} \mathrm{P}\right)^{4} \mathrm{D}_{1.5}$ & $167,339.24$ & $238,830.9$ & $1.78 \mathrm{E}+09$ & & TW \\
\hline 10 & & $1399.355(8)$ & $71,461.5$ & $1399.357(7)$ & -0.002 & $4 d^{9} 5 s 5 p$ & $\left({ }^{1} \mathrm{D}\right)^{2} \mathrm{~F}_{2.5}$ & - & $4 d^{9} 5 p^{2}$ & $\left({ }^{1} \mathrm{D}\right)^{2} \mathrm{D}_{1.5}$ & $176,090.19$ & $247,551.6$ & $2.30 \mathrm{E}+09$ & & TW \\
\hline 100 & & $1401.254(8)$ & $71,364.6$ & $1401.247(7)$ & 0.007 & $4 d^{9} 5 s 5 p$ & $\left({ }^{3} \mathrm{D}\right){ }^{4} \mathrm{~F}_{2.5}$ & - & $4 d^{9} 5 p^{2}$ & $\left({ }^{3} \mathrm{P}\right)^{4} \mathrm{D}_{1.5}$ & $167,465.9$ & $238,830.9$ & $4.84 \mathrm{E}+09$ & & TW \\
\hline 100 & & $1402.439(8)$ & $71,304.3$ & $1402.438(8)$ & 0.001 & $4 d^{9} 5 s 5 p$ & $\left({ }^{1} \mathrm{D}\right)^{2} \mathrm{D}_{2.5}$ & - & $4 d^{9} 5 p^{2}$ & $\left({ }^{3} \mathrm{P}\right)^{2} \mathrm{P}_{1.5}$ & $182,399.28$ & $253,703.7$ & $6.34 \mathrm{E}+09$ & & TW \\
\hline 280 & $\mathrm{w}$ & $1403.017(16)$ & $71,275.0$ & $1403.029(6)$ & -0.012 & $4 d^{10} 5 p$ & $\left({ }^{1} \mathrm{~S}\right)^{2} \mathrm{P}_{0.5}$ & - & $4 d^{10} 5 d$ & $\left({ }^{1} \mathrm{~S}\right)^{2} \mathrm{D}_{1.5}$ & $57,184.0$ & $128,458.36$ & $4.26 \mathrm{E}+09$ & & B, TW \\
\hline 150 & & $1404.342(8)$ & $71,207.7$ & $1404.343(7)$ & -0.001 & $4 d^{9} 5 s 5 p$ & $\left({ }^{3} \mathrm{D}\right)^{4} \mathrm{D}_{2.5}$ & - & $4 d^{9} 5 p^{2}$ & $\left({ }^{3} \mathrm{P}\right)^{2} \mathrm{D}_{1.5}$ & $174,496.6$ & $245,704.3$ & $3.40 \mathrm{E}+09$ & & TW \\
\hline 200 & & 1407.302(8) & $71,058.0$ & $1407.295(7)$ & 0.007 & $4 d^{9} 5 s 5 p$ & $\left({ }^{1} \mathrm{D}\right)^{2} \mathrm{D}_{2.5}$ & - & $4 d^{9} 5 p^{2}$ & $\left({ }^{3} \mathrm{P}\right)^{2} \mathrm{~F}_{3.5}$ & $182,399.28$ & $253,457.6$ & $4.50 \mathrm{E}+09$ & & TW \\
\hline 120 & & $1408.292(8)$ & $71,008.0$ & 1408.292(7) & 0.000 & $4 d^{9} 5 s 5 p$ & $\left({ }^{1} \mathrm{D}\right)^{2} \mathrm{~F}_{2.5}$ & - & $4 d^{9} 5 p^{2}$ & $\left({ }^{3} \mathrm{P}\right)^{4} \mathrm{~F}_{2.5}$ & $176,090.1,9$ & $247,098.2$ & $3.16 \mathrm{E}+09$ & & TW \\
\hline 2 & $\mathrm{f}$ & $1411.028(16)$ & $70,870.3$ & $1411.032(14)$ & -0.004 & $4 d^{9} 5 s 5 p$ & $\left({ }^{3} \mathrm{D}\right){ }^{4} \mathrm{~F}_{1.5}$ & - & $4 d^{9} 5 p^{2}$ & $\left({ }^{3} \mathrm{P}\right)^{4} \mathrm{D}_{0.5}$ & $170,813.7$ & $241,683.8$ & $3.00 \mathrm{E}+09$ & & TW \\
\hline 2 & $\mathrm{f}$ & $1413.821(16)$ & $70,730.3$ & 1413.813(9) & 0.008 & $4 d^{9} 5 s 5 p$ & $\left({ }^{3} D\right)^{4} \mathrm{~F}_{1.5}$ & - & $4 d^{9} 5 p^{2}$ & $\left({ }^{3} \mathrm{P}\right)^{2} \mathrm{~F}_{2.5}$ & $170,813.7$ & $241,544.42$ & $1.55 \mathrm{E}+09$ & & TW \\
\hline 150 & & $1418.119(8)$ & $70,515.9$ & 1418.112(6) & 0.007 & $4 d^{9} 5 s 5 p$ & $\left({ }^{3} D\right)^{4} D_{3.5}$ & - & $4 d^{9} 5 p^{2}$ & $\left({ }^{3} \mathrm{P}\right)^{4} \mathrm{~F}_{4.5}$ & $174,043.59$ & $244,559.9$ & $5.34 \mathrm{E}+09$ & & TW \\
\hline 5 & $\mathrm{f}$ & $1420.204(16)$ & $70,412.4$ & 1420.192(7) & 0.012 & $4 d^{9} 5 s 5 p$ & $\left({ }^{1} \mathrm{D}\right)^{2} \mathrm{~F}_{2.5}$ & - & $4 d^{9} 5 p^{2}$ & $\left({ }^{3} \mathrm{P}\right)^{4} \mathrm{P}_{2.5}$ & $176,090.19$ & $246,503.2$ & $4.82 \mathrm{E}+08$ & \# & TW \\
\hline 4 & $\mathrm{f}$ & $1421.105(16)$ & $70,367.8$ & $1421.098(15)$ & 0.007 & $4 d^{9} 5 s 5 p$ & $\left({ }^{3} \mathrm{D}\right){ }^{4} \mathrm{P}_{0.5}$ & - & $4 d^{9} 5 p^{2}$ & $\left({ }^{3} \mathrm{P}\right)^{4} \mathrm{D}_{0.5}$ & $171,315.7$ & $241,683.8$ & $1.38 \mathrm{E}+09$ & & TW \\
\hline 6 & & $1421.649(8)$ & $70,340.9$ & $1421.647(6)$ & 0.002 & $4 d^{10} 5 d$ & $\left({ }^{1} \mathrm{~S}\right)^{2} \mathrm{D}_{1.5}$ & - & $4 d^{9} 5 s 5 p$ & $\left({ }^{3} \mathrm{D}\right)^{2} \mathrm{~F}_{2.5}$ & $128,458.36$ & $198,799.3$ & $4.94 \mathrm{E}+09$ & & TW \\
\hline 100 & & $1425.676(8)$ & $70,142.2$ & $1425.673(8)$ & 0.003 & $4 d^{9} 5 s 5 p$ & $\left({ }^{1} \mathrm{D}\right)^{2} \mathrm{~F}_{3.5}$ & - & $4 d^{9} 5 p^{2}$ & $\left({ }^{3} \mathrm{P}\right)^{2} \mathrm{D}_{2.5}$ & $180,060.3$ & $250,202.6$ & $4.78 \mathrm{E}+09$ & & TW \\
\hline 35 & & $1430.130(8)$ & $69,923.7$ & $1430.127(7)$ & 0.003 & $4 d^{10} 5 \mathrm{~d}$ & $\left({ }^{1} \mathrm{~S}\right)^{2} \mathrm{D}_{1.5}$ & - & $4 d^{9} 5 s 5 p$ & $\left({ }^{3} \mathrm{D}\right)^{2} \mathrm{P}_{0.5}$ & $128,458.36$ & $198,382.2$ & $1.38 \mathrm{E}+09$ & & TW \\
\hline 280 & $\mathrm{w}$ & $1434.800(16)$ & $69,696.1$ & $1434.805(6)$ & -0.005 & $4 d^{10} 5 p$ & $\left({ }^{1} \mathrm{~S}\right)^{2} \mathrm{P}_{0.5}$ & - & $4 \mathrm{~d}^{10} 6 \mathrm{~s}$ & $\left({ }^{1} \mathrm{~S}\right)^{2} \mathrm{~S}_{0.5}$ & $57,184.0$ & $126,879.89$ & $1.08 \mathrm{E}+09$ & & B, TW \\
\hline 35 & $\mathrm{bl}$ & $1439.854(16)$ & $69,451.5$ & $1439.830(4)$ & 0.024 & $4 d^{9} 5 s^{2}$ & $\left({ }^{2} \mathrm{D}\right)^{2} \mathrm{D}_{2.5}$ & - & $4 d^{10} 5 f$ & $\left({ }^{1} \mathrm{~S}\right)^{2} \mathrm{~F}_{2.5}$ & $115,572.19$ & $185,024.81$ & $7.33 \mathrm{E}+06$ & & TW \\
\hline 100 & & $1440.281(8)$ & $69,430.9$ & $1440.291(7)$ & -0.010 & $4 d^{9} 5 s 5 p$ & $\left({ }^{1} \mathrm{D}\right)^{2} \mathrm{D}_{2.5}$ & - & $4 d^{9} 5 p^{2}$ & $\left({ }^{1} \mathrm{D}\right)^{2} \mathrm{~F}_{2.5}$ & $182,399.28$ & $251,829.7$ & $2.33 \mathrm{E}+09$ & & TW \\
\hline 200 & $\mathrm{bl}$ & $1442.512(16)$ & $69,323.5$ & $1442.507(5)$ & 0.005 & $4 d^{9} 5 s^{2}$ & $\left({ }^{2} \mathrm{D}\right)^{2} \mathrm{D}_{2.5}$ & - & $4 \mathrm{~d}^{10} 5 \mathrm{f}$ & $\left({ }^{1} \mathrm{~S}\right)^{2} \mathrm{~F}_{3.5}$ & $115,572.19$ & $184,895.95$ & $2.52 \mathrm{E}+08$ & & TW \\
\hline 110 & & $1447.387(8)$ & $69,090.0$ & 1447.401(6) & -0.014 & $4 d^{9} 5 s^{2}$ & $\left({ }^{2} D\right)^{2} D_{1.5}$ & - & $4 d^{9} 5 s 5 p$ & $\left({ }^{3} \mathrm{D}\right)^{2} \mathrm{P}_{1.5}$ & $122,419.73$ & $191,509.1$ & $1.09 \mathrm{E}+08$ & \# & B \\
\hline 100 & & $1467.495(8)$ & $68,143.3$ & $1467.504(6)$ & -0.009 & $4 d^{9} 5 s 5 p$ & $\left({ }^{3} \mathrm{D}\right){ }^{4} \mathrm{~F}_{3.5}$ & - & $4 d^{9} 5 p^{\frac{1}{2}}$ & $\left({ }^{3} \mathrm{P}\right)^{4} \mathrm{D}_{2.5}$ & $167,308.1$ & $235,451.0$ & $6.54 \mathrm{E}+09$ & & TW \\
\hline 120 & & 1468.172(8) & $68,111.9$ & $1468.175(6)$ & -0.003 & $4 d^{9} 5 s 5 p$ & $\left({ }^{3} \mathrm{D}\right){ }^{4} \mathrm{P}_{1.5}$ & - & $4 d^{9} 5 p^{2}$ & $\left({ }^{3} \mathrm{P}\right)^{4} \mathrm{D}_{2.5}$ & $167,339.24$ & $235,451.0$ & $3.47 \mathrm{E}+09$ & & TW \\
\hline 40 & & $1481.468(8)$ & $67,500.6$ & $1481.463(5)$ & 0.005 & $4 d^{9} 5 s 5 p$ & $\left({ }^{3} D^{4} \mathrm{D}_{3.5}\right.$ & - & $4 d^{9} 5 p^{2}$ & $\left({ }^{3} \mathrm{P}\right)^{2} \mathrm{~F}_{2.5}$ & $174,043.59$ & $241,544.42$ & $1.54 \mathrm{E}+09$ & & TW \\
\hline 8 & $\mathrm{f}$ & $1482.483(16)$ & $67,454.4$ & 1482.505(6) & -0.022 & $4 \mathrm{~d}^{10} 6 \mathrm{~s}$ & $\left({ }^{1} \mathrm{~S}\right)^{2} \mathrm{~S}_{0.5}$ & - & $4 d^{10} 8 p$ & $\left({ }^{1} \mathrm{~S}\right)^{2} \mathrm{P}_{1.5}$ & $126,879.89$ & $194,333.3$ & $8.27 \mathrm{E}+06$ & & B \\
\hline 300 & $\mathrm{w}$ & $1487.623(23)$ & $67,221.3$ & $1487.595(10)$ & 0.028 & $4 d^{9} 5 s 5 p$ & $\left({ }^{3} \mathrm{D}\right){ }^{4} \mathrm{~F}_{4.5}$ & - & $4 d^{9} 5 p^{2}$ & $\left({ }^{3} \mathrm{P}\right)^{4} \mathrm{D}_{3.5}$ & $168,947.6$ & $236,170.2$ & $1.06 \mathrm{E}+10$ & & B, TW \\
\hline 300 & $\mathrm{w}$ & $1487.623(23)$ & $67,221.3$ & 1487.623(5) & 0.000 & $4 d^{10} 5 p$ & $\left({ }^{1} \mathrm{~S}\right)^{2} \mathrm{P}_{1.5}$ & - & $4 d^{10} 5 d$ & $\left({ }^{1} \mathrm{~S}\right)^{2} \mathrm{D}_{2.5}$ & $61,527.0$ & $128,748.33$ & $8.44 \mathrm{E}+09$ & & TW \\
\hline 15 & & $1491.235(8)$ & $67,058.5$ & 1491.235(8) & 0.000 & $4 d^{10} 6 s$ & $\left({ }^{1} \mathrm{~S}\right)^{2} \mathrm{~S}_{0.5}$ & - & $4 d^{10} 8 p$ & $\left({ }^{1} \mathrm{~S}\right)^{2} \mathrm{P}_{0.5}$ & $126,879.89$ & $193,938.4$ & $5.84 \mathrm{E}+05$ & & B \\
\hline 5 & $\mathrm{f}$ & $1491.474(16)$ & $67,047.8$ & 1491.473(10) & 0.001 & $4 d^{9} 5 s 5 p$ & $\left({ }^{3} \mathrm{D}\right)^{4} \mathrm{D}_{2.5}$ & - & $4 d^{9} 5 p^{2}$ & $\left({ }^{3} \mathrm{P}\right)^{2} \mathrm{~F}_{2.5}$ & $174,496.6$ & $241,544.42$ & $2.20 \mathrm{E}+09$ & & TW \\
\hline 200 & $\mathrm{w}$ & $1494.066(16)$ & $66,931.4$ & $1494.068(5)$ & -0.002 & $4 d^{10} 5 p$ & $\left({ }^{1} \mathrm{~S}\right)^{2} \mathrm{P}_{1.5}$ & - & $4 d^{10} 5 d$ & $\left({ }^{1} \mathrm{~S}\right)^{2} \mathrm{D}_{1.5}$ & $61,527.0$ & $128,458.36$ & $7.21 \mathrm{E}+08$ & & B, TW \\
\hline 5 & & $1495.389(8)$ & $66,872.2$ & $1495.377(6)$ & 0.012 & $4 d^{10} 6 p$ & $\left({ }^{1} \mathrm{~S}\right)^{2} \mathrm{P}_{0.5}$ & - & $4 \mathrm{~d}^{10} 11 \mathrm{~s}$ & $\left({ }^{1} \mathrm{~S}\right)^{2} \mathrm{~S}_{0.5}$ & $144,589.32$ & $211,462.1$ & $2.09 \mathrm{E}+07$ & & B \\
\hline 20 & & $1505.020(8)$ & $66,444.3$ & 1505.021(7) & -0.001 & $4 d^{10} 5 d$ & $\left({ }^{1} \mathrm{~S}\right)^{2} \mathrm{D}_{1.5}$ & - & $4 d^{9} 5 s 5 p$ & $\left({ }^{3} \mathrm{D}\right)^{2} \mathrm{D}_{2.5}$ & $128,458.36$ & $194,902.6$ & $6.10 \mathrm{E}+08$ & & TW \\
\hline 40 & * & $1511.615(23)$ & $66,154.4$ & 1511.619(11) & -0.004 & $4 d^{9} 5 s 5 p$ & $\left({ }^{3} \mathrm{D}\right)^{4} \mathrm{D}_{1.5}$ & & $4 d^{9} 5 p^{2}$ & $\left({ }^{3} \mathrm{P}\right)^{4} \mathrm{~F}_{2.5}$ & $180,943.95$ & $247,098.2$ & $1.23 \mathrm{E}+09$ & & TW \\
\hline 40 & * & $1511.615(23)$ & $66,154.4$ & 1511.618(8) & -0.003 & $4 d^{10} 5 \mathrm{~d}$ & $\left({ }^{1} \mathrm{~S}\right)^{2} \mathrm{D}_{2.5}$ & - & $4 d^{9} 5 s 5 p$ & $\left({ }^{3} \mathrm{D}\right)^{2} \mathrm{D}_{2.5}$ & $128,748.33$ & $194,902.6$ & $1.91 \mathrm{E}+08$ & & TW \\
\hline 35 & & $1515.040(8)$ & $66,004.9$ & $1515.035(6)$ & 0.005 & $4 d^{9} 5 s 5 p$ & $\left.\left({ }^{1} \mathrm{D}\right)\right)^{2} \mathrm{D}_{1.5}$ & - & $4 d^{9} 5 p^{2}$ & $\left({ }^{3} \mathrm{P}\right)^{2} \mathrm{~F}_{2.5}$ & $175,539.35$ & $241,544.42$ & $2.10 \mathrm{E}+09$ & & TW \\
\hline 10 & & $1518.024(8)$ & $65,875.1$ & $1518.028(5)$ & -0.004 & $4 d^{10} 5 \mathrm{~d}$ & $\left({ }^{1} \mathrm{~S}\right)^{2} \mathrm{D}_{1.5}$ & - & $4 d^{10} 8 p$ & $\left({ }^{1} \mathrm{~S}\right)^{2} \mathrm{P}_{1.5}$ & $128,458.36$ & $194,333.3$ & $2.33 \mathrm{E}+07$ & \# & B \\
\hline 60 & & 1522.164(8) & $65,695.9$ & $1522.169(6)$ & -0.005 & $4 d^{9} 5 s 5 p$ & $\left({ }^{3} \mathrm{D}\right)^{4} \mathrm{D}_{3.5}$ & - & $4 d^{9} 5 p^{2}$ & $\left({ }^{1} \mathrm{D}\right)^{2} \mathrm{D}_{2.5}$ & $174,043.59$ & $239,739.3$ & $7.16 \mathrm{E}+08$ & & TW \\
\hline 25 & & $1524.750(8)$ & $65,584.5$ & $1524.740(6)$ & 0.010 & $4 d^{10} 5 d$ & $\left({ }^{1} S\right)^{2} D_{2.5}$ & - & $4 d^{10} 8 p$ & $\left({ }^{1} \mathrm{~S}\right)^{2} \mathrm{P}_{1.5}$ & $128,748.33$ & $194,333.3$ & $7.42 \mathrm{E}+07$ & & B \\
\hline 10 & & 1525.344(8) & $65,559.0$ & $1525.338(6)$ & 0.006 & $4 d^{9} 5 s 5 p$ & $\left({ }^{3} D\right)^{4} D_{1.5}$ & - & $4 d^{9} 5 p^{2}$ & $\left({ }^{3} \mathrm{P}\right)^{4} \mathrm{P}_{2.5}$ & $180,943.95$ & $246,503.2$ & $1.21 \mathrm{E}+08$ & \# & TW \\
\hline
\end{tabular}


Table 3. Cont

\begin{tabular}{|c|c|c|c|c|c|c|c|c|c|c|c|c|c|c|c|}
\hline$I_{\mathrm{obs}}{ }^{\mathrm{a}}$ & $\mathrm{ch}^{\mathrm{b}}$ & $\lambda_{\text {obs }}{ }^{\mathrm{c}} \AA$ & $\sigma_{\text {obs }} \mathrm{cm}^{-1}$ & $\lambda_{\text {Ritz }}{ }^{\mathrm{d}} \AA$ & $\Delta \lambda_{\text {O-Ritz }}$ e $\AA$ & & Classification $^{\mathrm{f}}$ & & & & $E_{\text {low }} \mathrm{cm}^{-1}$ & $E_{\text {upp }} \mathrm{cm}^{-1}$ & $g A^{\mathrm{h}} \mathrm{S}^{-1}$ & & Lin. $\operatorname{Ref}^{\mathrm{i}}$ \\
\hline 14 & & $1525.869(8)$ & $65,536.4$ & $1525.881(6)$ & -0.012 & $4 d^{10} 6 p$ & $\left({ }^{1} \mathrm{~S}\right)^{2} \mathrm{P}_{1.5}$ & - & $4 \mathrm{~d}^{10} 11 \mathrm{~s}$ & $\left({ }^{1} \mathrm{~S}\right)^{2} \mathrm{~S}_{0.5}$ & $145,926.21$ & $211,462.1$ & $3.95 \mathrm{E}+07$ & & B \\
\hline 100 & & $1526.000(8)$ & $65,530.8$ & $1525.996(7)$ & 0.004 & $4 d^{9} 5 \mathrm{~s} 5 \mathrm{p}$ & $\left({ }^{3} \mathrm{D}\right)^{4} \mathrm{D}_{1.5}$ & - & $4 d^{9} 5 p^{2}$ & $\left({ }^{3} \mathrm{P}\right)^{4} \mathrm{~F}_{1.5}$ & $180,943.95$ & $246,474.9$ & $7.67 \mathrm{E}+08$ & & TW \\
\hline 40 & & $1527.784(8)$ & $65,454.3$ & 1527.785(6) & -0.001 & $4 d^{9} 5 \mathrm{~s} 5 \mathrm{p}$ & $\left({ }^{1} \mathrm{D}\right)^{2} \mathrm{~F}_{2.5}$ & - & $4 d^{9} 5 p^{2}$ & $\left({ }^{3} \mathrm{P}\right)^{2} \mathrm{~F}_{2.5}$ & $176,090.19$ & $241,544.42$ & $7.27 \mathrm{E}+07$ & \# & TW \\
\hline 52 & & $1529.704(8)$ & $65,372.1$ & $1529.713(5)$ & -0.009 & $4 d^{9} 5 s^{2}$ & $\left({ }^{2} \mathrm{D}\right)^{2} \mathrm{D}_{2.5}$ & - & $4 d^{9} 5 s 5 p$ & $\left({ }^{3} \mathrm{D}\right)^{4} \mathrm{D}_{1.5}$ & $115,572.19$ & $180,943.95$ & 7.42E+06 & \# & B \\
\hline 260 & $\mathrm{w}$ & $1530.169(16)$ & $65,352.3$ & $1530.154(6)$ & 0.015 & $4 d^{10} 5 p$ & $\left({ }^{1} \mathrm{~S}\right)^{2} \mathrm{P}_{1.5}$ & - & $4 d^{10} 6 s$ & $\left({ }^{1} \mathrm{~S}\right)^{2} \mathrm{~S}_{0.5}$ & $61,527.0$ & $126,879.89$ & $1.82 \mathrm{E}+09$ & & B, TW \\
\hline 180 & $\mathrm{w}$ & $1532.926(16)$ & $65,234.7$ & $1532.902(6)$ & 0.024 & $4 d^{10} 5 p$ & $\left({ }^{1} \mathrm{~S}\right)^{2} \mathrm{P}_{0.5}$ & - & $4 d^{9} 5 s^{2}$ & $\left({ }^{2} \mathrm{D}\right)^{2} \mathrm{D}_{1.5}$ & $57,184.0$ & $122,419.73$ & $2.79 \mathrm{E}+09$ & & B, TW \\
\hline 5 & $\mathrm{f}$ & $1534.868(16)$ & $65,152.2$ & $1534.865(10)$ & 0.003 & $4 d^{9} 5 s 5 p$ & $\left({ }^{1} \mathrm{D}\right)^{2} \mathrm{D}_{2.5}$ & - & $4 d^{9} 5 p^{2}$ & $\left({ }^{1} \mathrm{D}\right)^{2} \mathrm{D}_{1.5}$ & $182,399.28$ & $247,551.6$ & $1.00 \mathrm{E}+08$ & \# & TW \\
\hline 48 & & $1547.300(8)$ & $64,628.7$ & $1547.288(6)$ & 0.012 & $4 d^{10} 6 s$ & $\left({ }^{1} \mathrm{~S}\right)^{2} \mathrm{~S}_{0.5}$ & - & $4 d^{9} 5 s 5 p$ & $\left({ }^{3} \mathrm{D}\right)^{2} \mathrm{P}_{1.5}$ & $126,879.89$ & $191,509.1$ & $1.64 \mathrm{E}+07$ & & B \\
\hline 25 & & 1579.653(8) & $63,305.0$ & $1579.654(7)$ & -0.001 & $4 d^{9} 5 s 5 p$ & $\left({ }^{1} \mathrm{D}\right)^{2} \mathrm{D}_{2.5}$ & - & $4 d^{9} 5 p^{2}$ & $\left({ }^{3} \mathrm{P}\right)^{2} \mathrm{D}_{1.5}$ & $182,399.28$ & $245,704.3$ & $4.09 \mathrm{E}+07$ & \# & TW \\
\hline 30 & & (1593.384) & $62,759.5$ & 1593.352(8) & & $4 d^{10} 5 \mathrm{~d}$ & $\left({ }^{1} \mathrm{~S}\right)^{2} \mathrm{D}_{2.5}$ & - & $4 d^{9} 5 s 5 p$ & $\left({ }^{3} \mathrm{D}\right)^{2} \mathrm{P}_{1.5}$ & $128,748.33$ & $191,509.1$ & $4.16 \mathrm{E}+06$ & \# & B \\
\hline 32 & & (1593.592) & $62,751.3$ & 1593.639(8) & & $4 d^{10} 6 p$ & $\left({ }^{1} \mathrm{~S}\right)^{2} \mathrm{P}_{0.5}$ & - & $4 d^{10} 9 \mathrm{~d}$ & $\left({ }^{1} \mathrm{~S}\right)^{2} \mathrm{D}_{1.5}$ & $144,589.32$ & $207,338.8$ & $8.02 \mathrm{E}+07$ & & B \\
\hline 78 & & $1597.329(8)$ & $62,604.5$ & $1597.314(4)$ & 0.015 & $4 d^{9} 5 s^{2}$ & $\left({ }^{2} \mathrm{D}\right)^{2} \mathrm{D}_{1.5}$ & - & $4 d^{10} 5 f$ & $\left({ }^{1} \mathrm{~S}\right)^{2} \mathrm{~F}_{2.5}$ & $122,419.73$ & $185,024.81$ & $1.92 \mathrm{E}+08$ & & B \\
\hline 50 & & $1600.535(8)$ & $62,479.1$ & $1600.535(6)$ & 0.000 & $4 d^{10} 6 p$ & $\left({ }^{1} \mathrm{~S}\right)^{2} \mathrm{P}_{0.5}$ & - & $4 \mathrm{~d}^{10} 10 \mathrm{~s}$ & $\left({ }^{1} \mathrm{~S}\right)^{2} \mathrm{~S}_{0.5}$ & $144,589.32$ & $207,068.43$ & $3.15 \mathrm{E}+07$ & & B \\
\hline 60 & $\mathrm{w}$ & (1605.211) & $62,297.1$ & 1605.251(6) & & $4 d^{9} 5 s^{2}$ & $\left({ }^{2} \mathrm{D}\right)^{2} \mathrm{D}_{2.5}$ & & $4^{10} 7 p$ & $\left({ }^{1} \mathrm{~S}\right)^{2} \mathrm{P}_{15}$ & $115,572.19$ & $177,867.74$ & $7.79 \mathrm{E}+05$ & \# & B \\
\hline 10 & & $1609.613(8)$ & $62,126.7$ & $1609.616(7)$ & -0.003 & $4 d^{9} 5 s 5 p$ & $\left({ }^{3} \mathrm{D}\right){ }^{4} \mathrm{D}_{3.5}$ & - & $4 d^{9} 5 p^{2}$ & $\left({ }^{3} \mathrm{P}\right)^{4} \mathrm{D}_{3.5}$ & $174,043.59$ & $236,170.2$ & $3.86 \mathrm{E}+08$ & & TW \\
\hline 400 & $\mathrm{w}$ & $1625.301(16)$ & $61,527.1$ & 1625.303(9) & -0.002 & $4 d^{10} 5 s$ & $\left({ }^{1} \mathrm{~S}\right)^{2} \mathrm{~S}_{0.5}$ & - & $4 d^{10} 5 p$ & $\left({ }^{1} \mathrm{~S}\right)^{2} \mathrm{P}_{1.5}$ & 0.0 & $61,527.0$ & $3.34 \mathrm{E}+09$ & & B, TW \\
\hline 60 & & $1627.249(8)$ & $61,453.4$ & 1627.247(8) & 0.002 & $4 d^{10} 6 p$ & $\left({ }^{1} \mathrm{~S}\right)^{2} \mathrm{P}_{1.5}$ & - & $4 d^{10} 9 d$ & $\left({ }^{1} \mathrm{~S}\right)^{2} \mathrm{D}_{2.5}$ & $145,926.21$ & $207,379.7$ & $1.37 \mathrm{E}+08$ & & B \\
\hline 49 & & $1628.330(8)$ & $61,412.6$ & 1628.331(8) & -0.001 & $4 d^{10} 6 \mathrm{p}$ & $\left({ }^{1} \mathrm{~S}\right)^{2} \mathrm{P}_{1.5}$ & - & $4 d^{10} 9 d$ & $\left({ }^{1} \mathrm{~S}\right)^{2} \mathrm{D}_{1.5}$ & $145,926.21$ & $207,338.8$ & $1.52 \mathrm{E}+07$ & & B \\
\hline 35 & & $1635.534(8)$ & $61,142.1$ & 1635.531(6) & 0.003 & $4 d^{10} 6 p$ & $\left({ }^{1} \mathrm{~S}\right)^{2} \mathrm{P}_{1.5}$ & - & $4 \mathrm{~d}^{10} 10 \mathrm{~s}$ & $\left({ }^{1} \mathrm{~S}\right)^{2} \mathrm{~S}_{0.5}$ & $145,926.21$ & $207,068.43$ & $5.93 \mathrm{E}+07$ & & B \\
\hline 180 & $\mathrm{w}$ & $1642.237(16)$ & $60,892.6$ & $1642.232(6)$ & 0.005 & $4 d^{10} 5 p$ & $\left({ }^{1} \mathrm{~S}\right)^{2} \mathrm{P}_{1.5}$ & - & $4 d^{9} 5 s^{2}$ & $(2 \mathrm{D})^{2} \mathrm{D}_{1.5}$ & $61,527.0$ & $122,419.73$ & $4.70 \mathrm{E}+08$ & & B, TW \\
\hline 65 & & 1667.581(8) & $59,967.1$ & 1667.579(5) & 0.002 & $4 d^{9} 5 s^{2}$ & $\left({ }^{2} \mathrm{D}\right)^{2} \mathrm{D}_{2.5}$ & - & $4 d^{9} 5 s 5 p$ & $\left({ }^{1} \mathrm{D}\right)^{2} \mathrm{D}_{1.5}$ & $115,572.19$ & $175,539.35$ & $2.38 \mathrm{E}+07$ & \# & B \\
\hline 60 & & $(1708.662)$ & $58,525.3$ & 1708.694(5) & & $4 d^{9} 5 s^{2}$ & $\left({ }^{2} \mathrm{D}\right)^{2} \mathrm{D}_{1.5}$ & - & $4 d^{9} 5 s 5 p$ & $\left({ }^{3} \mathrm{D}\right)^{4} \mathrm{D}_{1.5}$ & $122,419.73$ & $180,943.95$ & $2.48 \mathrm{E}+06$ & \# & B \\
\hline 400 & $\mathrm{w}$ & $1748.728(16)$ & $57,184.4$ & $1748.741(11)$ & -0.013 & $4 d^{10} 5 s$ & $\left({ }^{1} \mathrm{~S}\right)^{2} \mathrm{~S}_{0.5}$ & - & $4 d^{10} 5 p$ & $\left({ }^{1} \mathrm{~S}\right)^{2} \mathrm{P}_{0.5}$ & 0.0 & $57,184.0$ & $1.38 \mathrm{E}+09$ & & B, TW \\
\hline 14 & & $1757.432(8)$ & $56,901.2$ & $1757.433(8)$ & -0.001 & $4 d^{9} 5 s^{2}$ & $\left({ }^{2} \mathrm{D}\right)^{2} \mathrm{D}_{1.5}$ & - & $4 d^{9} 5 s 5 p$ & $\left({ }^{1} \mathrm{D}\right)^{2} \mathrm{P}_{0.5}$ & $122,419.73$ & $179,320.9$ & $6.87 \mathrm{E}+06$ & \# & B \\
\hline 68 & & $1767.840(8)$ & $56,566.2$ & 1767.832(5) & 0.008 & $4 d^{10} 5 \mathrm{~d}$ & $\left({ }^{1} \mathrm{~S}^{2} \mathrm{D}_{1.5}\right.$ & - & $4 \mathrm{~d}^{10} 5 \mathrm{f}$ & $\left({ }^{1} \mathrm{~S}\right)^{2} \mathrm{~F}_{2.5}$ & $128,458.36$ & $185,024.81$ & $6.40 \mathrm{E}+07$ & & B \\
\hline 53 & & $1776.943(8)$ & $56,276.4$ & $1776.941(5)$ & 0.002 & $4 d^{10} 5 \mathrm{~d}$ & $\left({ }^{1} \mathrm{~S}\right)^{2} \mathrm{D}_{2.5}$ & - & $4 d^{10} 5 f$ & $\left({ }^{1} \mathrm{~S}\right)^{2} \mathrm{~F}_{2.5}$ & $128,748.33$ & $185,024.81$ & $1.31 \mathrm{E}+07$ & & B \\
\hline 40 & & $1779.457(8)$ & $56,196.9$ & $1779.451(5)$ & 0.006 & $4 d^{9} 5 s^{2}$ & $\left({ }^{2} \mathrm{D}\right)^{2} \mathrm{D}_{1.5}$ & - & $4 d^{9} 5 s 5 p$ & $\left({ }^{1} \mathrm{D}\right)^{2} \mathrm{P}_{1.5}$ & $122,419.73$ & $178,616.85$ & $5.00 \mathrm{E}+06$ & \# & B \\
\hline 65 & & $1779.704(8)$ & $56,189.1$ & $1779.708(5)$ & -0.004 & $4 d^{10} 6 p$ & $\left({ }^{1} \mathrm{~S}\right)^{2} \mathrm{P}_{0.5}$ & - & $4 \mathrm{~d}^{10} 8 \mathrm{~d}$ & $\left({ }^{1} \mathrm{~S}\right)^{2} \mathrm{D}_{1.5}$ & $144,589.32$ & $200,778.32$ & $1.44 \mathrm{E}+08$ & & B \\
\hline 70 & & 1781.020(8) & $56,147.6$ & 1781.019(5) & 0.001 & $4 d^{10} 5 d$ & $\left({ }^{1} \mathrm{~S}\right)^{2} \mathrm{D}_{2.5}$ & - & $4 d^{10} 5 f$ & $\left({ }^{1} \mathrm{~S}\right)^{2} \mathrm{~F}_{3.5}$ & $128,748.33$ & $184,895.95$ & $2.40 \mathrm{E}+08$ & & B \\
\hline 50 & & 1792.962(8) & $55,773.63$ & $1792.968(6)$ & -0.006 & $4 d^{10} 6 p$ & $\left({ }^{1} \mathrm{~S}\right)^{2} \mathrm{P}_{0.5}$ & - & $4 d^{10} 9 \mathrm{~s}$ & $\left({ }^{1} \mathrm{~S}\right)^{2} \mathrm{~S}_{0.5}$ & $144,589.32$ & $200,362.77$ & $5.11 \mathrm{E}+07$ & & B \\
\hline 50 & & 1793.146(8) & $55,767.91$ & 1793.143(5) & 0.003 & $4 d^{9} 5 s^{2}$ & $\left({ }^{2} \mathrm{D}\right)^{2} \mathrm{D}_{1.5}$ & - & $4 d^{9} 5 s 5 p$ & $\left({ }^{3} \mathrm{D}\right)^{4} \mathrm{D}_{0.5}$ & $122,419.73$ & $178,187.72$ & $2.70 \mathrm{E}+05$ & \# & B \\
\hline 19 & & $1803.500(8)$ & $55,447.74$ & 1803.491(5) & 0.009 & $4 d^{9} 5 s^{2}$ & $\left({ }^{2} \mathrm{D}\right)^{2} \mathrm{D}_{1.5}$ & - & $4^{10} 7 p$ & $\left({ }^{1} \mathrm{~S}\right)^{2} \mathrm{P}_{1.5}$ & $122,419.73$ & $177,867.74$ & $9.39 \mathrm{E}+05$ & \# & B \\
\hline 60 & & 1821.158(8) & $54,910.12$ & 1821.169(5) & -0.011 & $4 d^{10} 6 p$ & $\left({ }^{1} \mathrm{~S}\right)^{2} \mathrm{P}_{1.5}$ & - & $4 d^{10} 8 d$ & $\left({ }^{1} \mathrm{~S}^{2} \mathrm{D}_{2.5}\right.$ & $145,926.21$ & $200,836.01$ & $2.44 \mathrm{E}+08$ & & B \\
\hline 41 & & $1823.097(8)$ & $54,851.72$ & $1823.084(5)$ & 0.013 & $4 d^{10} 6 p$ & $\left({ }^{1} \mathrm{~S}\right)^{2} \mathrm{P}_{1.5}$ & - & $4 d^{10} 8 \mathrm{~d}$ & $\left({ }^{1} \mathrm{~S}\right)^{2} \mathrm{D}_{1.5}$ & $145,926.21$ & $200,778.32$ & $2.70 \mathrm{E}+07$ & & B \\
\hline 29 & & $1823.363(8)$ & $54,843.71$ & $1823.365(6)$ & -0.002 & $4 d^{9} 5 s^{2}$ & $\left({ }^{2} \mathrm{D}\right)^{2} \mathrm{D}_{1.5}$ & - & $4^{10} 7 p$ & $\left({ }^{1} \mathrm{~S}\right)^{2} \mathrm{P}_{0.5}$ & $122,419.73$ & $177,263.38$ & $8.19 \mathrm{E}+06$ & \# & B \\
\hline 60 & & 1837.006(8) & $54,436.40$ & 1837.001(6) & 0.005 & $4 d^{10} 6 p$ & $\left({ }^{1} \mathrm{~S}\right)^{2} \mathrm{P}_{1.5}$ & - & $4 d^{10} 9 s$ & $\left({ }^{1} \mathrm{~S}\right)^{2} \mathrm{~S}_{0.5}$ & $145,926.21$ & $200,362.77$ & $9.55 \mathrm{E}+07$ & & B \\
\hline 80 & $\mathrm{w}$ & $1850.280(16)$ & $54,045.9$ & $1850.303(8)$ & -0.023 & $4 d^{10} 5 p$ & $\left({ }^{1} \mathrm{~S}\right)^{2} \mathrm{P}_{1.5}$ & - & $4 d^{9} 5 s^{2}$ & $(2 \mathrm{D})^{2} \mathrm{D}_{2.5}$ & $61,527.0$ & $115,572.19$ & $2.28 \mathrm{E}+08$ & & B, TW \\
\hline 70 & & $1882.547(8)$ & $53,119.52$ & $1882.544(6)$ & 0.003 & $4 d^{9} 5 s^{2}$ & $\left({ }^{2} \mathrm{D}\right)^{2} \mathrm{D}_{1.5}$ & - & $4 d^{9} 5 s 5 p$ & $\left({ }^{1} \mathrm{D}\right)^{2} \mathrm{D}_{15}$ & $122,419.73$ & $175,539.35$ & $1.09 \mathrm{E}+07$ & \# & B \\
\hline 13 & & $1905.284(8)$ & $52,485.61$ & $1905.285(6)$ & -0.001 & $4 \mathrm{~d}^{10} 5 \mathrm{~d}$ & $\left({ }^{1} \mathrm{~S}\right)^{2} \mathrm{D}_{1.5}$ & - & $4 d^{9} 5 s 5 p$ & $\left({ }^{3} \mathrm{D}\right)^{4} \mathrm{D}_{1.5}$ & $128,458.36$ & $180,943.95$ & $2.37 \mathrm{E}+05$ & \# & B \\
\hline
\end{tabular}


Table 3. Cont

\begin{tabular}{|c|c|c|c|c|c|c|c|c|c|c|c|c|c|c|c|}
\hline$I_{\mathrm{obs}}{ }^{\mathrm{a}}$ & $\mathrm{ch}^{\mathrm{b}}$ & $\lambda_{\text {obs }}{ }^{\mathrm{c}} \AA$ & $\sigma_{\mathrm{obs}} \mathrm{cm}^{-1}$ & $\lambda_{\text {Ritz }}{ }^{\mathrm{d}} \AA$ & $\Delta \lambda_{\text {O-Ritz }}{ }^{\mathrm{e}} \AA$ & & Classification $^{\mathrm{f}}$ & & & & $E_{\text {low }} \mathrm{cm}^{-1}$ & $E_{\text {upp }} \mathrm{cm}^{-1}$ & $g A^{\mathrm{h}} \mathrm{S}^{-1}$ & & Lin. $\operatorname{Ref}^{\mathrm{i}}$ \\
\hline 19 & & 1915.881(8) & $52,195.31$ & $1915.870(6)$ & 0.011 & $4 d^{10} 5 d$ & $\left({ }^{1} \mathrm{~S}\right)^{2} \mathrm{D}_{2.5}$ & - & $4 d^{9} 5 s 5 p$ & $\left({ }^{3} \mathrm{D}\right)^{4} \mathrm{D}_{1.5}$ & $128,748.33$ & $180,943.95$ & $1.26 \mathrm{E}+05$ & \# & B \\
\hline 50 & & $1923.343(10)$ & $51,992.8$ & $1923.343(10)$ & 0.000 & $4 \mathrm{~d}^{10} 4 \mathrm{f}$ & $\left({ }^{1} \mathrm{~S}\right)^{2} \mathrm{~F}_{2.5}$ & - & $4 d^{10} 9 g$ & $\left({ }^{1} \mathrm{~S}\right)^{2} \mathrm{G}_{3.5}$ & $161,974.14$ & $213,966.94$ & $2.19 \mathrm{E}+08$ & sh & \\
\hline 46 & & $1923.654(10)$ & $51,984.4$ & 1923.662(7) & -0.008 & $4 d^{10} 4 f$ & $\left({ }^{1} \mathrm{~S}\right)^{2} \mathrm{~F}_{3.5}$ & - & $4 d^{10} 9 g$ & $\left({ }^{1}\right)^{2} \mathrm{G}_{4.5}$ & $161,982.00$ & $213,966.18$ & $2.84 \mathrm{E}+08$ & sh & \\
\hline 75 & & $1931.728(8)$ & $51,767.12$ & $1931.731(7)$ & -0.003 & $4 d^{9} 5 s^{2}$ & $\left({ }^{2} \mathrm{D}\right)^{2} \mathrm{D}_{2.5}$ & - & $4 d^{9} 5 s 5 p$ & $\left({ }^{3} \mathrm{D}\right){ }^{4} \mathrm{P}_{1.5}$ & $115,572.19$ & $167,339.24$ & $1.33 \mathrm{E}+07$ & \# & B \\
\hline 58 & & (1932.89) & $51,736.00$ & 1932.854(8) & & $4 d^{10} 6 s$ & $\left({ }^{1} \mathrm{~S}\right)^{2} \mathrm{~S}_{0.5}$ & - & $4 d^{9} 5 s 5 p$ & $\left({ }^{1} \mathrm{D}\right)^{2} \mathrm{P}_{1.5}$ & $126,879.89$ & $178,616.85$ & $3.74 \mathrm{E}+06$ & & B \\
\hline 28 & & 1949.021(8) & $51,307.81$ & $1949.020(6)$ & 0.001 & $4 d^{10} 6 s$ & $\left({ }^{1} \mathrm{~S}\right)^{2} \mathrm{~S}_{0.5}$ & - & $4 d^{9} 5 s 5 p$ & $\left({ }^{3} D\right)^{4} D_{0.5}$ & $126,879.89$ & $178,187.72$ & $2.11 \mathrm{E}+06$ & & B \\
\hline 38 & & $1961.245(8)$ & $50,988.02$ & 1961.252(6) & -0.007 & $4 d^{10} 6 s$ & $\left({ }^{1} \mathrm{~S}\right)^{2} \mathrm{~S}_{0.5}$ & - & $4 \mathrm{~d}^{10} 7 \mathrm{p}$ & $\left({ }^{1} \mathrm{~S}\right)^{2} \mathrm{P}_{1.5}$ & $126,879.89$ & $177,867.74$ & $3.29 \mathrm{E}+05$ & \# & B \\
\hline 75 & & (1965.976) & $50,865.32$ & 1966.083(11) & & $4 d^{10} 5 d$ & $\left({ }^{1} \mathrm{~S}\right)^{2} \mathrm{D}_{1.5}$ & - & $4 d^{9} 5 s 5 p$ & $\left({ }^{1} \mathrm{D}\right)^{2} \mathrm{P}_{0.5}$ & $128,458.36$ & $179,320.9$ & $1.19 \mathrm{E}+05$ & \# & B \\
\hline 30 & & $1984.780(8)$ & $50,383.42$ & 1984.777(6) & 0.003 & $4 d^{10} 6 s$ & $\left({ }^{1} \mathrm{~S}\right)^{2} \mathrm{~S}_{0.5}$ & - & $4 d^{10} 7 \mathrm{p}$ & $\left({ }^{1} \mathrm{~S}\right)^{2} \mathrm{P}_{0.5}$ & $126,879.89$ & $177,263.38$ & $1.38 \mathrm{E}+05$ & \# & B \\
\hline 10 & & 1993.680(8) & $50,158.50$ & $1993.680(6)$ & 0.000 & $4 d^{10} 5 d$ & $\left({ }^{1} S\right)^{2} \mathrm{D}_{1.5}$ & - & $4 d^{9} 5 s 5 p$ & $\left({ }^{1} \mathrm{D}\right)^{2} \mathrm{P}_{1.5}$ & $128,458.36$ & $178,616.85$ & $4.67 \mathrm{E}+05$ & \# & B \\
\hline 66 & & $2004.620(20)$ & $49,868.6$ & 2004.624(8) & -0.004 & $4 d^{10} 5 d$ & $\left({ }^{1} \mathrm{~S}\right)^{2} \mathrm{D}_{2.5}$ & - & $4 d^{9} 5 s 5 p$ & $\left({ }^{1} \mathrm{D}\right)^{2} \mathrm{P}_{1.5}$ & $128,748.33$ & $178,616.85$ & $3.64 \mathrm{E}+07$ & & B \\
\hline 36 & & $2010.200(20)$ & $49,730.2$ & 2010.235(8) & -0.035 & $4 d^{10} 5 d$ & $\left({ }^{1} \mathrm{~S}\right)^{2} \mathrm{D}_{1.5}$ & - & $4 d^{9} 5 s 5 p$ & $\left({ }^{3} D\right)^{4} D_{0.5}$ & $128,458.36$ & $178,187.72$ & $2.68 \mathrm{E}+07$ & \# & B \\
\hline 63 & & $2023.260(20)$ & $49,409.3$ & $2023.255(7)$ & 0.005 & $4 d^{10} 5 d$ & $\left({ }^{1} \mathrm{~S}\right)^{2} \mathrm{D}_{1.5}$ & - & $4 d^{10} 7 \mathrm{p}$ & $\left({ }^{1} \mathrm{~S}\right)^{2} \mathrm{P}_{1.5}$ & $128,458.36$ & $177,867.74$ & $8.57 \mathrm{E}+06$ & \# & B \\
\hline 76 & & 2035.190(20) & $49,119.7$ & 2035.201(8) & -0.011 & $4 d^{10} 5 \mathrm{~d}$ & $\left({ }^{1} S\right)^{2} D_{2.5}$ & - & $4 d^{10} 7 \mathrm{p}$ & $\left({ }^{1} \mathrm{~S}\right)^{2} \mathrm{P}_{1.5}$ & $128,748.33$ & $177,867.74$ & $5.75 \mathrm{E}+07$ & & B \\
\hline 76 & & $2048.310(20)$ & $48,805.1$ & 2048.313(8) & -0.003 & $4 d^{10} 5 d$ & $\left({ }^{1} \mathrm{~S}\right)^{2} \mathrm{D}_{1.5}$ & - & $4 d^{10} 7 p$ & $\left({ }^{1} \mathrm{~S}\right)^{2} \mathrm{P}_{0.5}$ & $128,458.36$ & $177,263.38$ & $1.84 \mathrm{E}+07$ & \# & B \\
\hline 74 & & $2051.070(20)$ & $48,739.4$ & 2051.092(8) & -0.022 & $4 \mathrm{~d}^{10} 4 \mathrm{f}$ & $\left({ }^{1} \mathrm{~S}\right)^{2} \mathrm{~F}_{2.5}$ & - & $4 d^{10} 8 g$ & $\left({ }^{1} \mathrm{~S}\right)^{2} \mathrm{G}_{3.5}$ & $161,974.14$ & $210,713.04$ & $3.39 \mathrm{E}+08$ & & B \\
\hline 78 & & $2051.410(20)$ & $48,731.3$ & 2051.423(9) & -0.013 & $4 \mathrm{~d}^{10} 4 \mathrm{f}$ & $\left({ }^{1} \mathrm{~S}\right)^{2} \mathrm{~F}_{3.5}$ & - & $4 \mathrm{~d}^{10} 8 \mathrm{~g}$ & $\left({ }^{1} \mathrm{~S}\right)^{2} \mathrm{G}_{3.5}$ & $161,982.00$ & $210,713.04$ & $1.25 \mathrm{E}+07$ & & B \\
\hline 10 & & $2136.480(20)$ & $46,791.2$ & 2136.488(9) & -0.008 & $4 d^{10} 5 \mathrm{~d}$ & $\left({ }^{1} \mathrm{~S}\right)^{2} \mathrm{D}_{2.5}$ & - & $4 d^{9} 5 s 5 p$ & $\left({ }^{1} \mathrm{D}\right)^{2} \mathrm{D}_{1.5}$ & $128,748.33$ & $175,539.35$ & $3.77 \mathrm{E}+05$ & \# & B \\
\hline 81 & & $(2154.04)$ & $46,409.8$ & 2154.039(11) & & $4 d^{9} 5 s^{2}$ & $\left({ }^{2} \mathrm{D}\right)^{2} \mathrm{D}_{2.5}$ & - & $4 \mathrm{~d}^{10} 4 \mathrm{f}$ & $\left({ }^{1} \mathrm{~S}\right)^{2} \mathrm{~F}_{3.5}$ & $115,572.19$ & $161,982.00$ & $1.48 \mathrm{E}+08$ & & B \\
\hline 80 & & $2154.400(20)$ & $46,402.0$ & $2154.404(10)$ & -0.004 & $4 d^{9} 5 s^{2}$ & $\left({ }^{2} \mathrm{D}\right)^{2} \mathrm{D}_{2.5}$ & - & $4 \mathrm{~d}^{10} 4 \mathrm{f}$ & $\left({ }^{1} \mathrm{~S}\right)^{2} \mathrm{~F}_{2.5}$ & $115,572.19$ & $161,974.14$ & $8.14 \mathrm{E}+06$ & & B \\
\hline 77 & & 2199.550(20) & $45,449.7$ & 2199.558(13) & -0.008 & $4 d^{10} 6 p$ & $\left({ }^{1} \mathrm{~S}\right)^{2} \mathrm{P}_{0.5}$ & - & $4 d^{10} 7 d$ & $\left({ }^{1} \mathrm{~S}\right)^{2} \mathrm{D}_{1.5}$ & $144,589.32$ & $190,038.8$ & $3.15 \mathrm{E}+08$ & & B \\
\hline 42 & & $(2201.47)$ & $45,410.0$ & $2201.686(19)$ & & $4 d^{10} 4 f$ & $\left({ }^{1} \mathrm{~S}\right)^{2} \mathrm{~F}_{2.5}$ & - & $4 d^{10} 9 d$ & $\left({ }^{1} \mathrm{~S}\right)^{2} \mathrm{D}_{2.5}$ & $161,974.14$ & $207,379.7$ & $2.98 \mathrm{E}+05$ & & B \\
\hline 10 & & (2203.54) & $45,367.4$ & 2203.671(18) & & $4 d^{10} 4 f$ & $\left({ }^{1} \mathrm{~S}\right)^{2} \mathrm{~F}_{2.5}$ & - & $4 d^{10} 9 d$ & $\left({ }^{1} \mathrm{~S}^{2} \mathrm{D}_{1.5}\right.$ & $161,974.14$ & $207,338.8$ & $4.11 \mathrm{E}+06$ & & B \\
\hline 45 & & $2225.480(20)$ & $44,920.2$ & $2225.512(11)$ & -0.032 & $4 d^{9} 5 s^{2}$ & $\left({ }^{2} \mathrm{D}\right)^{2} \mathrm{D}_{1.5}$ & - & $4 d^{9} 5 s 5 p$ & $\left({ }^{3} D\right)^{4} P_{1.5}$ & $122,419.73$ & $167,339.24$ & $8.27 \mathrm{E}+05$ & $\#$ & B \\
\hline 75 & & $2232.170(20)$ & $44,785.5$ & $2232.188(13)$ & -0.018 & $4 d^{10} 6 p$ & $\left({ }^{1} \mathrm{~S}\right)^{2} \mathrm{P}_{0.5}$ & & $4 \mathrm{~d}^{10} 8 \mathrm{~s}$ & $\left({ }^{1} \mathrm{~S}\right)^{2} \mathrm{~S}_{0.5}$ & $144,589.32$ & $189,374.5$ & $9.32 \mathrm{E}+07$ & & B \\
\hline 77 & & $2261.230(20)$ & $44,210.0$ & $2261.227(19)$ & 0.003 & $4 d^{10} 6 p$ & $\left({ }^{1} \mathrm{~S}\right)^{2} \mathrm{P}_{1.5}$ & - & $4 d^{10} 7 \mathrm{~d}$ & $\left({ }^{1} \mathrm{~S}\right)^{2} \mathrm{D}_{2.5}$ & $145,926.21$ & $190,136.3$ & $5.25 \mathrm{E}+08$ & & B \\
\hline 74 & & $2266.230(20)$ & $44,112.5$ & $2266.226(14)$ & 0.004 & $4 d^{10} 6 p$ & $\left({ }^{1} \mathrm{~S}\right)^{2} \mathrm{P}_{1.5}$ & - & $4 d^{10} 7 d$ & $\left({ }^{1} \mathrm{~S}\right)^{2} \mathrm{D}_{1.5}$ & $145,926.21$ & $190,038.8$ & $5.80 \mathrm{E}+07$ & & B \\
\hline 78 & & $2272.370(20)$ & $43,993.3$ & $2272.417(9)$ & -0.047 & $4 d^{10} 4 f$ & $\left({ }^{1} \mathrm{~S}\right)^{2} \mathrm{~F}_{2.5}$ & - & $4 d^{10} 7 g$ & $\left({ }^{1} \mathrm{~S}\right)^{2} \mathrm{G}_{3.5}$ & $161,974.14$ & $205,966.56$ & $5.74 \mathrm{E}+08$ & & B \\
\hline 80 & & $2272.810(20)$ & $43,984.8$ & $2272.812(13)$ & -0.002 & $4 d^{10} 4 f$ & $\left({ }^{1} \mathrm{~S}\right)^{2} \mathrm{~F}_{3.5}$ & - & $4 \mathrm{~d}^{10} 7 \mathrm{~g}$ & $\left({ }^{1} \mathrm{~S}\right)^{2} \mathrm{G}_{4.5}$ & $161,982.00$ & $205,966.76$ & $7.44 \mathrm{E}+08$ & & B \\
\hline 81 & & $2300.890(20)$ & $43,448.1$ & $2300.878(14)$ & 0.012 & $4 d^{10} 6 p$ & $\left({ }^{1} \mathrm{~S}\right)^{2} \mathrm{P}_{1.5}$ & - & $4 d^{10} 8 s$ & $\left({ }^{1} \mathrm{~S}\right)^{2} \mathrm{~S}_{0.5}$ & $145,926.21$ & $189,374.5$ & $1.72 \mathrm{E}+08$ & & B \\
\hline 74 & & $2527.380(20)$ & $39,554.8$ & $2527.403(11)$ & -0.023 & $4 d^{9} 5 s^{2}$ & $\left({ }^{2} \mathrm{D}\right)^{2} \mathrm{D}_{1.5}$ & - & $4 d^{10} 4 f$ & $\left({ }^{1} \mathrm{~S}\right)^{2} \mathrm{~F}_{2.5}$ & $122,419.73$ & $161,974.14$ & $1.45 \mathrm{E}+09$ & & B \\
\hline 10 & & $(2572.42)$ & $38,862.3$ & $2572.446(15)$ & & $4 d^{10} 4 f$ & $\left({ }^{1} \mathrm{~S}\right)^{2} \mathrm{~F}_{2.5}$ & - & $4 d^{10} 8 \mathrm{~d}$ & $\left({ }^{1} \mathrm{~S}\right)^{2} \mathrm{D}_{2.5}$ & $161,974.14$ & $200,836.01$ & $4.91 \mathrm{E}+05$ & & B \\
\hline 30 & & (2572.94) & $38,854.4$ & $2572.966(17)$ & & $4 \mathrm{~d}^{10} 4 \mathrm{f}$ & $\left({ }^{1} \mathrm{~S}\right)^{2} \mathrm{~F}_{3.5}$ & - & $4 d^{10} 8 \mathrm{~d}$ & $\left({ }^{1} \mathrm{~S}\right)^{2} \mathrm{D}_{2.5}$ & $161,982.00$ & $200,836.01$ & $9.82 \mathrm{E}+06$ & & B \\
\hline 22 & & (2576.15) & $38,806.0$ & $2576.270(15)$ & & $4 \mathrm{~d}^{10} 4 \mathrm{f}$ & $\left({ }^{1} \mathrm{~S}\right)^{2} \mathrm{~F}_{2.5}$ & - & $4 d^{10} 8 \mathrm{~d}$ & $\left({ }^{1} \mathrm{~S}\right)^{2} \mathrm{D}_{1.5}$ & $161,974.14$ & $200,778.32$ & $6.77 \mathrm{E}+06$ & & B \\
\hline 85 & & $2725.460(20)$ & $36,680.2$ & 2725.462(19) & -0.002 & $4 d^{10} 4 f$ & $\left({ }^{1} \mathrm{~S}\right)^{2} \mathrm{~F}_{2.5}$ & - & $4 d^{10} 6 g$ & $\left({ }^{1} \mathrm{~S}\right)^{2} \mathrm{G}_{3.5}$ & $161,974.14$ & $198,654.3$ & $1.13 \mathrm{E}+09$ & & B \\
\hline 86 & * & $2726.07(6)$ & $36,672.0$ & $2726.046(23)$ & 0.02 & $4 d^{10} 4 f$ & $\left({ }^{1} \mathrm{~S}\right)^{2} \mathrm{~F}_{3.5}$ & - & $4 d^{10} 6 g$ & $\left({ }^{1} \mathrm{~S}\right)^{2} \mathrm{G}_{3.5}$ & $161,982.00$ & $198,654.3$ & $4.18 \mathrm{E}+07$ & & B \\
\hline 86 & * & $2726.07(6)$ & $36,672.0$ & $2726.07(6)$ & 0.000 & $4 \mathrm{~d}^{10} 4 \mathrm{f}$ & $\left({ }^{1} \mathrm{~S}\right)^{2} \mathrm{~F}_{3.5}$ & - & $4 d^{10} 6 g$ & $\left({ }^{1} \mathrm{~S}\right)^{2} \mathrm{G}_{4.5}$ & $161,982.00$ & $198,654.0$ & $1.46 \mathrm{E}+09$ & & B \\
\hline 26 & & $(2923.41)$ & $34,196.62$ & 2923.23(3) & & $4 d^{10} 7 p$ & $\left({ }^{1} \mathrm{~S}\right)^{2} \mathrm{P}_{0.5}$ & - & $4 d^{10} 11 \mathrm{~s}$ & $\left({ }^{1} \mathrm{~S}\right)^{2} \mathrm{~S}_{0.5}$ & $177,263.38$ & $211,462.1$ & $8.81 \mathrm{E}+06$ & & B \\
\hline 80 & & $2982.800(20)$ & $33,515.77$ & 2982.799(14) & 0.001 & $4 d^{10} 5 d$ & $\left({ }^{1} \mathrm{~S}\right)^{2} \mathrm{D}_{1.5}$ & - & $4 \mathrm{~d}^{10} 4 \mathrm{f}$ & $\left({ }^{1} \mathrm{~S}\right)^{2} \mathrm{~F}_{2.5}$ & $128,458.36$ & $161,974.14$ & $2.16 \mathrm{E}+09$ & & $\mathrm{~s}$ \\
\hline
\end{tabular}


Table 3. Cont

\begin{tabular}{|c|c|c|c|c|c|c|c|c|c|c|c|c|c|c|}
\hline$I_{\mathrm{obs}}{ }^{\mathrm{a}}$ & $\mathrm{ch}^{\mathrm{b}}$ & $\lambda_{\text {obs }}{ }^{\mathrm{c}} \AA$ & $\sigma_{\mathrm{obs}} \mathrm{cm}^{-1}$ & $\lambda_{\text {Ritz }}{ }^{\mathrm{d}} \AA$ & $\Delta \lambda_{\text {O-Ritz }}{ }^{\mathrm{e}} \AA$ & & Classification $^{\mathrm{f}}$ & & & & $E_{\text {low }} \mathrm{cm}^{-1}$ & $E_{\text {upp }} \mathrm{cm}^{-1}$ & $g A^{\mathrm{h}} \mathrm{S}^{-1}$ & Lin. $\operatorname{Ref}^{\mathrm{i}}$ \\
\hline 82 & & $3008.080(20)$ & $33,234.11$ & $3008.120(15)$ & -0.040 & $4 d^{10} 5 d$ & $\left({ }^{1} \mathrm{~S}\right)^{2} \mathrm{D}_{2.5}$ & - & $4 d^{10} 4 f$ & $\left({ }^{1} \mathrm{~S}\right)^{2} \mathrm{~F}_{3.5}$ & $128,748.33$ & $161,982.00$ & $4.91 \mathrm{E}+09$ & $\mathrm{~s}$ \\
\hline 77 & & $(3008.76)$ & $33,226.60$ & $3008.832(17)$ & & $4 d^{10} 5 d$ & $\left({ }^{1} \mathrm{~S}\right)^{2} \mathrm{D}_{2.5}$ & - & $4 \mathrm{~d}^{10} 4 \mathrm{f}$ & $\left({ }^{1} \mathrm{~S}\right)^{2} \mathrm{~F}_{2.5}$ & $128,748.33$ & $161,974.14$ & $2.44 \mathrm{E}+08$ & B \\
\hline 45 & & (3293.56) & $30,353.54$ & 3293.51(3) & & $4 d^{9} 5 s^{2}$ & $\left({ }^{2} \mathrm{D}\right)^{2} \mathrm{D}_{2.5}$ & - & $4 d^{10} 6 p$ & $\left({ }^{1} \mathrm{~S}\right)^{2} \mathrm{P}_{1.5}$ & $115,572.19$ & $145,926.21$ & $4.41 \mathrm{E}+06$ & B \\
\hline 21 & & $3438.970(20)$ & $29,070.14$ & $3438.960(18)$ & 0.010 & $4 d^{10} 5 f$ & $\left({ }^{1} \mathrm{~S}\right)^{2} \mathrm{~F}_{3.5}$ & - & $4 d^{10} 9 g$ & $\left({ }^{1} \mathrm{~S}\right)^{2} \mathrm{G}_{4.5}$ & $184,895.95$ & $213,966.18$ & $2.06 \mathrm{E}+08$ & B \\
\hline 37 & & (3551.03) & $28,152.80$ & $3550.84(6)$ & & $4 \mathrm{~d}^{10} 4 \mathrm{f}$ & $\left({ }^{1} \mathrm{~S}\right)^{2} \mathrm{~F}_{3.5}$ & - & $4 \mathrm{~d}^{10} 7 \mathrm{~d}$ & $\left({ }^{1} \mathrm{~S}\right)^{2} \mathrm{D}_{2.5}$ & $161,982.00$ & $190,136.3$ & $1.84 \mathrm{E}+07$ & B \\
\hline 28 & & $(3562.35)$ & $28,063.34$ & $3562.18(5)$ & & $4 \mathrm{~d}^{10} 4 \mathrm{f}$ & $\left({ }^{1} \mathrm{~S}\right)^{2} \mathrm{~F}_{2.5}$ & - & $4 d^{10} 7 d$ & $\left({ }^{1} \mathrm{~S}\right)^{2} \mathrm{D}_{1.5}$ & $161,974.14$ & $190,038.8$ & $1.27 \mathrm{E}+07$ & B \\
\hline 30 & * & $3640.69(10)$ & $27,459.5$ & $3640.69(10)$ & 0.00 & $4 d^{10} 5 g$ & $\left({ }^{1} \mathrm{~S}\right)^{2} \mathrm{G}_{4.5}$ & - & $4 d^{10} 9 \mathrm{~h}$ & $\left({ }^{1} \mathrm{~S}\right)^{2} \mathrm{H}_{5.5}$ & $186,528.26$ & $213,987.75$ & $1.37 \mathrm{E}+08$ & B \\
\hline 30 & * & $3640.69(10)$ & $27,459.5$ & $3640.64(8)$ & 0.05 & $4 d^{10} 5 g$ & $\left({ }^{1} \mathrm{~S}\right)^{2} \mathrm{G}_{3.5}$ & - & $4 d^{10} 9 \mathrm{~h}$ & $\left({ }^{1} \mathrm{~S}\right)^{2} \mathrm{H}_{4.5}$ & $186,527.40$ & $213,987.3$ & $1.11 \mathrm{E}+08$ & B \\
\hline 30 & * & $3640.69(10)$ & $27,459.5$ & $3640.75(8)$ & -0.06 & $4 d^{10} 5 g$ & $\left({ }^{1} \mathrm{~S}\right)^{2} \mathrm{G}_{4.5}$ & - & $4 d^{10} 9 h$ & $\left({ }^{1} \mathrm{~S}\right)^{2} \mathrm{H}_{4.5}$ & $186,528.26$ & $213,987.3$ & 2.53E+06 & B \\
\hline 91 & & $3853.010(20)$ & $25,946.38$ & 3853.001(17) & 0.009 & $4 d^{10} 6 p$ & $\left({ }^{1} \mathrm{~S}\right)^{2} \mathrm{P}_{0.5}$ & - & $4 \mathrm{~d}^{10} 6 \mathrm{~d}$ & $\left({ }^{1} \mathrm{~S}\right)^{2} \mathrm{D}_{1.5}$ & $144,589.32$ & $170,535.76$ & $1.15 \mathrm{E}+09$ & B \\
\hline 65 & & $3872.630(20)$ & $25,814.93$ & $3872.630(20)$ & 0.000 & $4 d^{10} 5 f$ & $\left({ }^{1} \mathrm{~S}\right)^{2} \mathrm{~F}_{3.5}$ & - & $4 d^{10} 8 g$ & $\left({ }^{1} \mathrm{~S}\right)^{2} \mathrm{G}_{4.5}$ & $184,895.95$ & $210,710.88$ & $3.11 \mathrm{E}+08$ & B \\
\hline 45 & & $3891.740(20)$ & $25,688.17$ & $3891.731(19)$ & 0.009 & $4 d^{10} 5 f$ & $\left({ }^{1} \mathrm{~S}\right)^{2} \mathrm{~F}_{2.5}$ & - & $4 d^{10} 8 g$ & $\left({ }^{1} \mathrm{~S}\right)^{2} \mathrm{G}_{3.5}$ & $185,024.81$ & $210,713.04$ & $2.42 \mathrm{E}+08$ & B \\
\hline 86 & & $(4023.82)$ & $24,844.98$ & 4023.77(3) & & $4 d^{10} 6 p$ & $\left({ }^{1} \mathrm{~S}\right)^{2} \mathrm{P}_{0.5}$ & - & $4 d^{10} 7 \mathrm{~s}$ & $\left({ }^{1} \mathrm{~S}\right)^{2} \mathrm{~S}_{0.5}$ & $144,589.32$ & $169,434.59$ & $2.29 \mathrm{E}+08$ & B \\
\hline 90 & & $4032.320(20)$ & $24,792.61$ & $4032.322(20)$ & -0.002 & $4 d^{10} 6 p$ & $\left({ }^{1} \mathrm{~S}\right)^{2} \mathrm{P}_{1.5}$ & - & $4 d^{10} 6 \mathrm{~d}$ & $\left({ }^{1} \mathrm{~S}\right)^{2} \mathrm{D}_{2.5}$ & $145,926.21$ & $170,718.81$ & $1.81 \mathrm{E}+09$ & $\mathrm{~s}$ \\
\hline 88 & & $4062.310(20)$ & $24,609.59$ & $4062.316(17)$ & -0.006 & $4 d^{10} 6 p$ & $\left({ }^{1} \mathrm{~S}\right)^{2} \mathrm{P}_{1.5}$ & - & $4 \mathrm{~d}^{10} 6 \mathrm{~d}$ & $\left({ }^{1} \mathrm{~S}\right)^{2} \mathrm{D}_{1.5}$ & $145,926.21$ & $170,535.76$ & $1.97 \mathrm{E}+08$ & B \\
\hline 81 & & $4071.640(20)$ & $24,553.20$ & $4071.629(19)$ & 0.011 & $4 \mathrm{~d}^{10} 4 \mathrm{f}$ & $\left({ }^{1} \mathrm{~S}\right)^{2} \mathrm{~F}_{2.5}$ & - & $4 d^{10} 5 g$ & $\left({ }^{1} \mathrm{~S}\right)^{2} \mathrm{G}_{3.5}$ & $161,974.14$ & $186,527.40$ & $2.96 \mathrm{E}+09$ & B \\
\hline 92 & & $4072.780(20)$ & $24,546.32$ & $4072.790(19)$ & -0.010 & $4 \mathrm{~d}^{10} 4 \mathrm{f}$ & $\left({ }^{1} \mathrm{~S}\right)^{2} \mathrm{~F}_{3.5}$ & - & $4 d^{10} 5 g$ & $\left({ }^{1} \mathrm{~S}\right)^{2} \mathrm{G}_{4.5}$ & $161,982.00$ & $186,528.26$ & $1.10 \mathrm{E}+08$ & B \\
\hline 40 & * & $4128.42(10)$ & $24,215.5$ & 4128.42(10) & 0.00 & $4 d^{10} 5 g$ & $\left({ }^{1} \mathrm{~S}\right)^{2} \mathrm{G}_{4.5}$ & - & $4 d^{10} 8 \mathrm{~h}$ & $\left({ }^{1} \mathrm{~S}\right)^{2} \mathrm{H}_{5.5}$ & $186,528.26$ & $210,743.8$ & 2.43E +08 & B \\
\hline 40 & * & $4128.42(10)$ & $24,215.5$ & $4128.35(8)$ & 0.07 & $4 d^{10} 5 g$ & $\left({ }^{1} \mathrm{~S}\right)^{2} \mathrm{G}_{3.5}$ & - & $4 \mathrm{~d}^{10} 8 \mathrm{~h}$ & $\left({ }^{1} \mathrm{~S}\right)^{2} \mathrm{H}_{4.5}$ & $186,527.40$ & $210,743.3$ & $1.98 \mathrm{E}+08$ & B \\
\hline 40 & * & $4128.42(10)$ & $24,215.5$ & $4128.50(8)$ & -0.08 & $4 d^{10} 5 g$ & $\left({ }^{1} \mathrm{~S}\right)^{2} \mathrm{G}_{4.5}$ & - & $4 \mathrm{~d}^{10} 8 \mathrm{~h}$ & $\left({ }^{1} \mathrm{~S}\right)^{2} \mathrm{H}_{4.5}$ & $186,528.26$ & $210,743.3$ & $4.50 \mathrm{E}+06$ & B \\
\hline 38 & & $(4233.56)$ & $23,614.13$ & $4233.50(6)$ & & $4 d^{10} 6 \mathrm{~d}$ & $\left({ }^{1} \mathrm{~S}\right)^{2} \mathrm{D}_{2.5}$ & - & $4 d^{10} 8 p$ & $\left({ }^{1} \mathrm{~S}\right)^{2} \mathrm{P}_{1.5}$ & $170,718.81$ & $194,333.3$ & $7.87 \mathrm{E}+06$ & B \\
\hline 64 & & (4250.94) & $23,517.59$ & $4251.42(4)$ & & $4 d^{10} 7 p$ & $\left({ }^{1} \mathrm{~S}\right)^{2} \mathrm{P}_{0.5}$ & - & $4 \mathrm{~d}^{10} 8 \mathrm{~d}$ & $\left({ }^{1} \mathrm{~S}\right)^{2} \mathrm{D}_{1.5}$ & $177,263.38$ & $200,778.32$ & $7.93 \mathrm{E}+07$ & B \\
\hline 88 & & $4252.600(20)$ & $23,508.41$ & $4252.605(20)$ & -0.005 & $4 d^{10} 6 p$ & $\left({ }^{1} \mathrm{~S}\right)^{2} \mathrm{P}_{1.5}$ & - & $4 \mathrm{~d}^{10} 7 \mathrm{~s}$ & $\left({ }^{1} S^{2}\right)^{2} \mathrm{~S}_{0.5}$ & $145,926.21$ & $169,434.59$ & $3.93 \mathrm{E}+08$ & B \\
\hline 80 & & $(4252.91)$ & $23,506.69$ & 4252.95(4) & & $4 d^{9} 5 s^{2}$ & $\left({ }^{2} D\right)^{2} D_{1.5}$ & - & $4 d^{10} 6 p$ & $\left({ }^{1} \mathrm{~S}\right)^{2} \mathrm{P}_{1.5}$ & $122,419.73$ & $145,926.21$ & $1.51 \mathrm{E}+07$ & B \\
\hline 40 & & (4328.03) & $23,098.71$ & $4327.90(5)$ & & $4 d^{10} 7 p$ & $\left({ }^{1} \mathrm{~S}\right)^{2} \mathrm{P}_{0.5}$ & - & $4 d^{10} 9 s$ & $\left({ }^{1} \mathrm{~S}\right)^{2} \mathrm{~S}_{0.5}$ & $177,263.38$ & $200,362.77$ & $2.32 \mathrm{E}+07$ & B \\
\hline 12 & & $4352.620(20)$ & $22,968.21$ & $4352.609(19)$ & 0.011 & $4 d^{10} 7 p$ & $\left({ }^{1} \mathrm{~S}\right)^{2} \mathrm{P}_{1.5}$ & - & $4 d^{10} 8 \mathrm{~d}$ & $\left({ }^{1} \mathrm{~S}\right)^{2} \mathrm{D}_{2.5}$ & $177,867.74$ & $200,836.01$ & $1.48 \mathrm{E}+08$ & B \\
\hline 2 & & $4363.560(20)$ & $22,910.63$ & $4363.569(19)$ & -0.009 & $4 d^{10} 7 p$ & $\left({ }^{1} \mathrm{~S}\right)^{2} \mathrm{P}_{1.5}$ & - & $4 \mathrm{~d}^{10} 8 \mathrm{~d}$ & $\left({ }^{1} \mathrm{~S}\right)^{2} \mathrm{D}_{1.5}$ & $177,867.74$ & $200,778.32$ & $1.65 \mathrm{E}+07$ & B \\
\hline 50 & & $(4444.36)$ & $22,494.11$ & $4444.18(4)$ & & $4 d^{10} 7 \mathrm{p}$ & $\left.{ }^{1} \mathrm{~S}\right)^{2} \mathrm{P}_{1.5}$ & - & $4 d^{10} 9 \mathrm{~s}$ & $\left({ }^{1} S\right)^{2} S_{0.5}$ & $177,867.74$ & $200,362.77$ & $4.83 \mathrm{E}+07$ & B \\
\hline 22 & & (4479.97) & $22,315.32$ & $4480.24(8)$ & & $4 d^{10} 5 f$ & $\left({ }^{1} \mathrm{~S}\right)^{2} \mathrm{~F}_{2.5}$ & - & $4 d^{10} 9 \mathrm{~d}$ & $\left({ }^{1} \mathrm{~S}\right)^{2} \mathrm{D}_{1.5}$ & $185,024.81$ & $207,338.8$ & $5.53 \mathrm{E}+06$ & B \\
\hline 87 & & (4509.78) & $22,167.81$ & $4509.42(4)$ & & $4 d^{9} 5 s^{2}$ & $\left({ }^{2} D\right)^{2} \mathrm{D}_{1.5}$ & - & $4 d^{10} 6 p$ & $\left({ }^{1} \mathrm{~S}\right)^{2} \mathrm{P}_{0.5}$ & $122,419.73$ & $144,589.32$ & $6.28 \mathrm{E}+07$ & B \\
\hline 73 & * & $4744.58(6)$ & $21,070.8$ & $4744.62(4)$ & -0.04 & $4 d^{10} 5 f$ & $\left({ }^{1} \mathrm{~S}\right)^{2} \mathrm{~F}_{3.5}$ & - & $4 \mathrm{~d}^{10} 7 \mathrm{~g}$ & $\left({ }^{1} \mathrm{~S}\right)^{2} \mathrm{G}_{3.5}$ & $184,895.95$ & $205,966.56$ & $1.44 \mathrm{E}+07$ & B \\
\hline 73 & * & $4744.58(6)$ & $21,070.8$ & $4744.58(5)$ & 0.00 & $4 d^{10} 5 f$ & $\left({ }^{1} \mathrm{~S}\right)^{2} \mathrm{~F}_{3.5}$ & - & $4 d^{10} 7 \mathrm{~g}$ & $\left({ }^{1} \mathrm{~S}\right)^{2} \mathrm{G}_{4.5}$ & $184,895.95$ & $205,966.76$ & $5.05 \mathrm{E}+08$ & B \\
\hline 63 & & $4773.830(20)$ & $20,941.69$ & $4773.815(19)$ & 0.015 & $4 \mathrm{~d}^{10} 5 \mathrm{f}$ & $\left({ }^{1} \mathrm{~S}\right)^{2} \mathrm{~F}_{2.5}$ & - & $4 \mathrm{~d}^{10} 7 \mathrm{~g}$ & $\left({ }^{1} \mathrm{~S}\right)^{2} \mathrm{G}_{3.5}$ & $185,024.81$ & $205,966.56$ & $3.93 \mathrm{E}+08$ & B \\
\hline 44 & * & $5130.85(10)$ & $19,484.5$ & $5130.85(10)$ & 0.00 & $4 d^{10} 5 g$ & $\left({ }^{1} \mathrm{~S}\right)^{2} \mathrm{G}_{4.5}$ & - & $4 \mathrm{~d}^{10} 7 \mathrm{~h}$ & $\left({ }^{1} \mathrm{~S}\right)^{2} \mathrm{H}_{5.5}$ & $186,528.26$ & $206,012.78$ & $5.20 \mathrm{E}+08$ & B \\
\hline 44 & * & $5130.85(10)$ & $19,484.5$ & $5130.75(8)$ & 0.10 & $4 d^{10} 5 g$ & $\left({ }^{1} \mathrm{~S}\right)^{2} \mathrm{G}_{3.5}$ & - & $4 \mathrm{~d}^{10} 7 \mathrm{~h}$ & $\left({ }^{1} \mathrm{~S}\right)^{2} \mathrm{H}_{4.5}$ & $186,527.40$ & $206,012.3$ & $4.23 \mathrm{E}+08$ & B \\
\hline 44 & * & $5130.85(10)$ & $19,484.5$ & $5130.98(8)$ & -0.13 & $4 d^{10} 5 g$ & $\left({ }^{1} \mathrm{~S}\right)^{2} \mathrm{G}_{4.5}$ & - & $4 \mathrm{~d}^{10} 7 \mathrm{~h}$ & $\left({ }^{1} \mathrm{~S}\right)^{2} \mathrm{H}_{4.5}$ & $186,528.26$ & $206,012.3$ & $9.62 \mathrm{E}+06$ & B \\
\hline
\end{tabular}


Table 3. Cont.

\begin{tabular}{|c|c|c|c|c|c|c|c|c|c|c|c|c|c|c|}
\hline$I_{\text {obs }}{ }^{a}$ & $\mathrm{ch}^{\mathrm{b}}$ & $\lambda_{\text {obs }}{ }^{c} \AA$ & $\sigma_{\text {obs }} \mathrm{cm}^{-1}$ & $\lambda_{\text {Ritz }}{ }^{\mathrm{d}} \AA$ & $\Delta \lambda_{\text {O-Ritz }}{ }^{\mathrm{e}} \AA$ & & Classification $^{\mathrm{f}}$ & & & & $E_{\text {low }} \mathrm{cm}^{-1}$ & $E_{\text {upp }} \mathrm{cm}^{-1}$ & $g A^{\mathrm{h}} \mathrm{S}^{-1}$ & Lin. Ref ${ }^{i}$ \\
\hline 72 & & (5248.77) & $19,046.78$ & $5248.90(6)$ & & $4 \mathrm{~d}^{10} 6 \mathrm{~s}$ & $\left({ }^{1} \mathrm{~S}\right)^{2} \mathrm{~S}_{0.5}$ & - & $4 d^{10} 6 p$ & $\left({ }^{1} \mathrm{~S}\right)^{2} \mathrm{P}_{1.5}$ & $126,879.89$ & $145,926.21$ & $4.79 \mathrm{E}+08$ & $\mathrm{~S}$ \\
\hline 70 & & (5644.96) & $17,710.00$ & $5645.14(7)$ & & $4 d^{10} 6 s$ & $\left({ }^{1} \mathrm{~S}\right)^{2} \mathrm{~S}_{0.5}$ & - & $4 d^{10} 6 \mathrm{p}$ & $\left({ }^{1} \mathrm{~S}\right)^{2} \mathrm{P}_{0.5}$ & $126,879.89$ & $144,589.32$ & $1.96 \mathrm{E}+08$ & B \\
\hline 76 & & (5722.71) & $17,469.39$ & $5723.22(7)$ & & $4 d^{10} 5 \mathrm{~d}$ & $\left({ }^{1} \mathrm{~S}\right)^{2} \mathrm{D}_{1.5}$ & - & $4 d^{10} 6 p$ & $\left({ }^{1} \mathrm{~S}\right)^{2} \mathrm{P}_{1.5}$ & $128,458.36$ & $145,926.21$ & $2.50 \mathrm{E}+07$ & B \\
\hline 70 & & (5819.41) & $17,179.11$ & $5819.83(8)$ & & $4 d^{10} 5 \mathrm{~d}$ & $\left({ }^{1} \mathrm{~S}\right)^{2} \mathrm{D}_{25}$ & - & $4 d^{10} 6 \mathrm{p}$ & $\left({ }^{1} \mathrm{~S}\right)^{2} \mathrm{P}_{15}$ & $128,748.33$ & $145,926.21$ & $3.39 \mathrm{E}+08$ & B \\
\hline 40 & & (6197.72) & $16,130.50$ & 6197.54(9) & & $4 \mathrm{~d}^{10} 5 \mathrm{~d}$ & $\left({ }^{1} \mathrm{~S}^{2} \mathrm{D}_{1.5}\right.$ & - & $4 \mathrm{~d}^{10} 6 \mathrm{p}$ & $\left({ }^{1} \mathrm{~S}\right)^{2} \mathrm{P}_{0.5}$ & $128,458.36$ & $144,589.32$ & $1.02 \mathrm{E}+08$ & B \\
\hline 10 & & (6520.50) & $15,332.01$ & $6519.8(5)$ & & $4 d^{10} 6 g$ & $\left({ }^{1} \mathrm{~S}\right)^{2} \mathrm{G}_{4.5}$ & - & $4 d^{10} 9 h$ & $\left({ }^{1} \mathrm{~S}\right)^{2} \mathrm{H}_{5.5}$ & $198,654.0$ & $213,987.75$ & $1.49 \mathrm{E}+08$ & B \\
\hline
\end{tabular}

a Observed relative intensities on an arbitrary scale (1-400) for the blackening of the lines on the photographic plates. Response functions of the instruments were not taken into account. ${ }^{b}$ Character of the observed line encoded as follows: $\mathrm{w}$-wide line; f-faint line; sh- shaded line; ${ }^{*}$ - intensity shared by two or more transitions. ${ }^{\mathrm{c}}$ Observed and Ritz wavelengths are given in standard air for wavenumber $\sigma$ between $5000 \mathrm{~cm}^{-1}$ and $50,000 \mathrm{~cm}^{-1}$ and in vacuum ouside of this range. The uncertainty (standard deviation) in the last digit is given in parentheses for both $\lambda_{\text {obs }}$ and $\lambda_{\text {Ritz, }}(\lambda)$ denotes values not included in the level optimization. ${ }^{\mathrm{d}}$ Ritz wavelengths and their uncertainties were determined in the least-squares level optimization procedure LOPT [15]. ${ }^{\mathrm{e}}$ Difference between observed and Ritz wavelength. If this column is blank, the line was excluded from the level optimization because its observed wavelength deviates from the Ritz value by more than our given uncertainty. ${ }^{\mathrm{f}}$ Classification specifies the lower and upper levels of the transition. ${ }^{\mathrm{h}}$ Weighted transition probability values $(g=2 J$ upper +1 is statistical weight of the upper level). If marked as \# then the given $g A$ values are too unreliable for the transitions whose cancellation factor $|C F|<0.10$ in our calculations with Cowan's code [12]. ${ }^{1}$ Reference to the source: B-Bhatia et al. [6]; B, TW-Wavelength from this work; K—Kaufman et al. [7]; R-Ryabtsev et al. [9]; S-Skočić et al. [10]; TW-this work. 


\subsection{The $4 d^{10} 5 s-\left[4 d^{10} n p\right]$ Transition Array}

The resonance transitions $4 \mathrm{~d}^{10} 5 \mathrm{~s}-4 \mathrm{~d}^{10} 5 \mathrm{p}$ were first reported by Rao [1], and confirmed by all other workers [2-5]. We observed these two lines in our indium spectra with high intensity. They were the main reference in establishing the In III ionization characteristics. Bhatia [6] reported the levels of $4 \mathrm{~d}^{10} n \mathrm{p}(n=5-9)$. We agreed with Bhatia's analysis only up to $4 \mathrm{~d}^{10} 8 \mathrm{p}$. The $4 \mathrm{~d}^{10} 5 \mathrm{~s}-4 \mathrm{~d}^{10} 9 \mathrm{p}$ transitions could not be seen in our spectra. The reported level value of $4 \mathrm{~d}^{10} 9 \mathrm{p}{ }^{2} \mathrm{P}_{3 / 2,1 / 2}$ at $201,180.3 \mathrm{~cm}^{-1}$ did not fit in our least squares fitted parametric calculations. Our predicted values were found to be at $203,388.5 \mathrm{~cm}^{-1}$ and $203,556.3 \mathrm{~cm}^{-1}$ for ${ }^{2} \mathrm{P}_{3 / 2}$ and ${ }^{2} \mathrm{P}_{1 / 2}$, respectively. A plot of the energy differences between observed and Hartree-Fock (HF) calculated values of $4 \mathrm{~d}^{10} n \mathrm{p}(n=5-9){ }^{2} \mathrm{P}_{3 / 2}$ series is shown in Figure 2, and it is evident from this figure that the reported value for $4 \mathrm{~d}^{10} 9 \mathrm{p}$ levels shows an irregular behavior. Therefore, this reported level seems to be doubtful. We did not find any alternative value as $4 \mathrm{~d}^{10} 9 \mathrm{p}$ transitions were too weak to be observed on our plates.

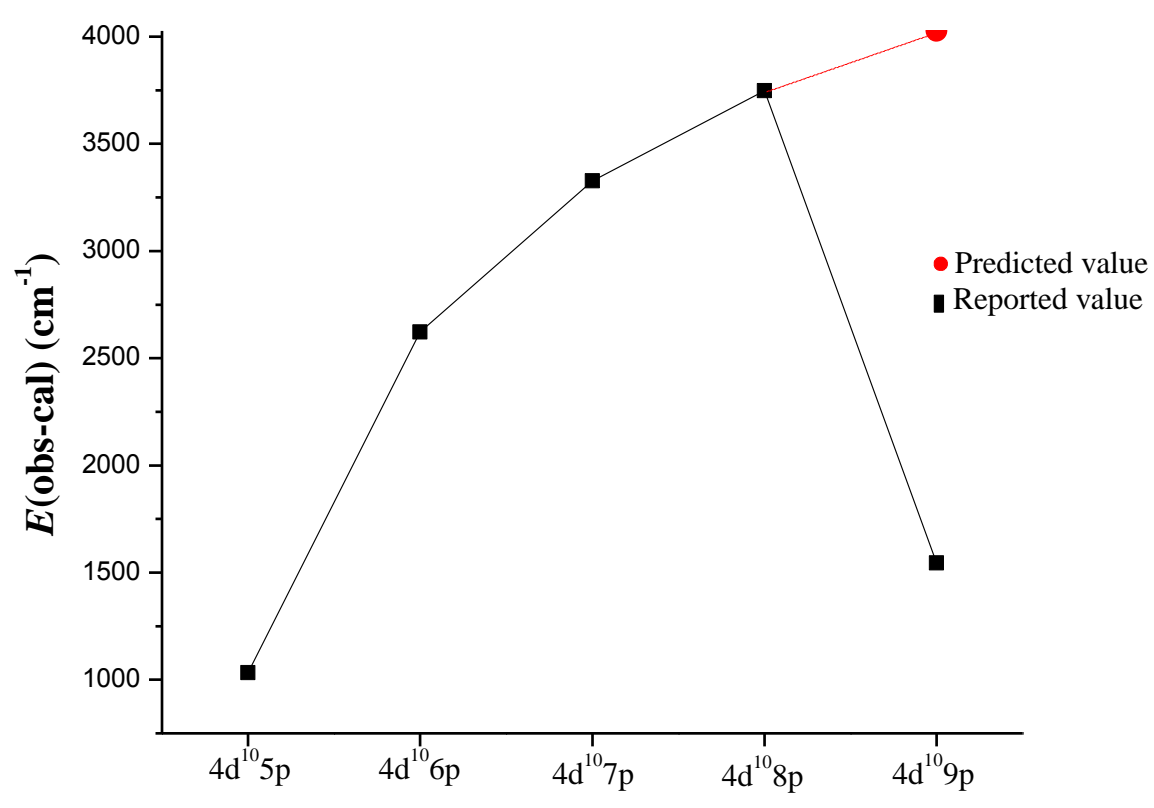

Figure 2. A plot of the observed and calculated energy difference in $4 \mathrm{~d}^{10} n \mathrm{p}$ series of In III.

\subsection{The $4 d^{10} n p-\left[4 d^{10}\{n s+n d\}+4 d^{9} 5 s^{2}\right]$ Transition Array}

The second excitation, $4 \mathrm{~d}^{10} 5 \mathrm{p}-\left[4 \mathrm{~d}^{10}(6 \mathrm{~s}+5 \mathrm{~d})+4 \mathrm{~d}^{9} 5 \mathrm{~s}^{2}\right]$ transitions, is also observed to be quite strong. In the $4 \mathrm{~d}^{10} n \mathrm{~s}$ series, we observed transitions $4 \mathrm{~d}^{10} 5 \mathrm{p}-4 \mathrm{~d}^{10} n \mathrm{~s}(n=6-8)$ and $4 \mathrm{~d}^{10} 6 \mathrm{p}-4 \mathrm{~d}^{10} n \mathrm{~s}$ $(n=9-12)$, and, in the $4 \mathrm{~d}^{10} n \mathrm{~d}$ series, three transitions are possible between each of the $4 \mathrm{~d}^{10} n \mathrm{p}-4 \mathrm{~d}^{10} n \mathrm{~d}$ configurations out of which two transitions, namely ${ }^{2} \mathrm{P}_{1 / 2}{ }^{2} \mathrm{D}_{3 / 2}$ and ${ }^{2} \mathrm{P}_{3 / 2}{ }^{2} \mathrm{D}_{5 / 2}$, were observed to be quite strong, while the third transition, ${ }^{2} \mathrm{P}_{3 / 2}{ }^{2} \mathrm{D}_{3 / 2}$, was predicted to be weak in the series. All these three transitions were observed in $4 \mathrm{~d}^{10}[5 \mathrm{p}-\mathrm{nd}(\mathrm{n}=5-7)]$. Thus, we confirmed the levels of the $4 \mathrm{~d}^{10} n \mathrm{~s}$ $(n=6-12)$ and $4 \mathrm{~d}^{10} n \mathrm{~d}(n=5-7)$ configurations. The transitions from $4 \mathrm{~d}^{10} n \mathrm{~d}(n=8,9)$ to $4 \mathrm{~d}^{10} 5 \mathrm{p}$ were not observed on our plates. However, these transitions were reported by Bhatia [6]. We examined these levels and found their scaling factor to be quite regular. Secondly, a similar plot as in Figure 2 with the average energy difference between the calculated and observed values shows a regular behavior for the $4 \mathrm{~d}^{10} n \mathrm{~s}$ and $4 \mathrm{~d}^{10} n \mathrm{~d}$ series (Figure 3). Although we could not confirm the levels of the $4 \mathrm{~d}^{10} n \mathrm{~d}$ $(n=8,9)$ configurations, on the basis of their regularity, we included them in Table 2 for the sake of completeness. 


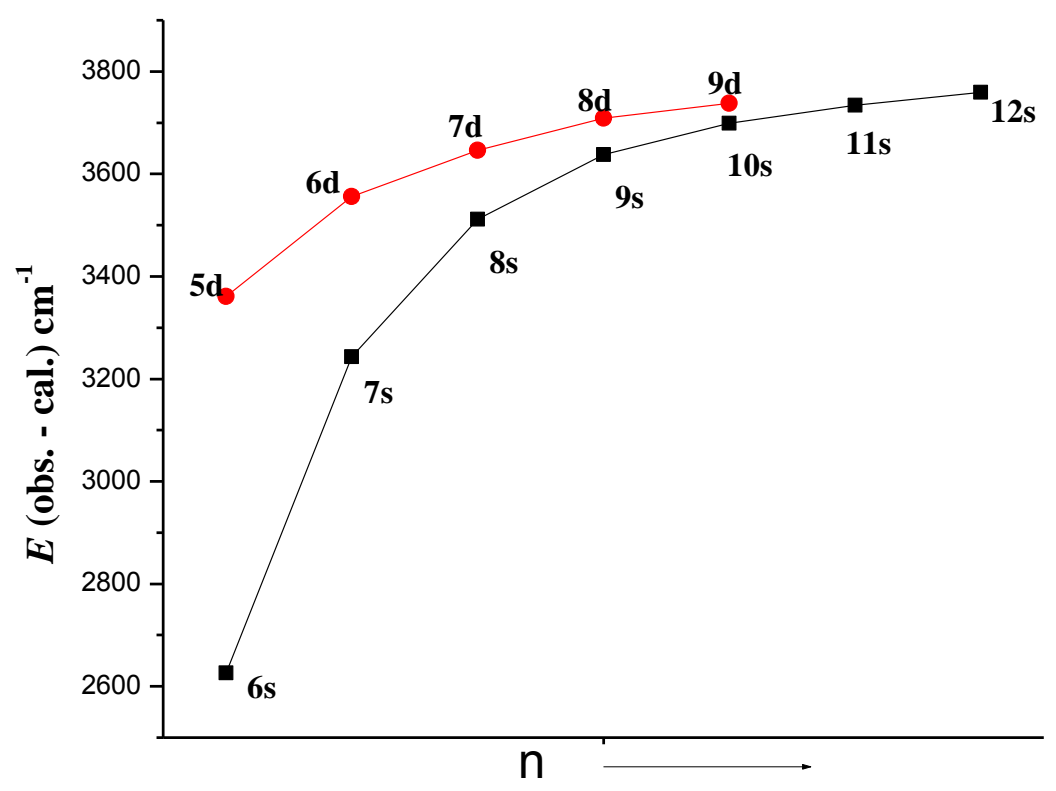

Figure 3. A plot $E$ (obs-cal) for the $4 \mathrm{~d}^{10} n \mathrm{~s}$ and $4 \mathrm{~d}^{10} n \mathrm{~d}$ series in In III.

The other configuration $4 \mathrm{~d}^{9} 5 \mathrm{~s}^{2}$ in even parity system has two inverted ${ }^{2} \mathrm{D}$ levels having the same energy range as the $4 \mathrm{~d}^{10} 5 \mathrm{~d}{ }^{2} \mathrm{D}$ levels. Both ${ }^{2} \mathrm{D}$ levels of these two configurations interact with each other. As a result of this interaction, $4 d^{10} 5 p-4 d^{9} 5 s^{2}$ transitions are observed. Further confirmation of these two levels was made by the observed transitions from the levels of the $4 d^{9} 5 s 5 p$ configuration that will be discussed later.

\subsection{The $4 d^{10}(n f+n g+n h)$ Configurations}

The $4 \mathrm{~d}^{10} 5 \mathrm{~d}-4 \mathrm{~d}^{10} 4 \mathrm{f}$ transitions lie beyond our wavelength region of investigation (above $2080 \AA$ ); therefore, we could not confirm them experimentally in the present work. However, these levels were well established by Nodwell [4] along with levels of the $4 \mathrm{~d}^{10} n \mathrm{~g}(n=5-7)$ series by observing transitions from $4 \mathrm{~d}^{10} 4 \mathrm{f}$. The repeated appearance of the $4 \mathrm{~d}^{10} 4 \mathrm{f}^{2} \mathrm{~F}_{5 / 2,7 / 2}$ interval in transitions from the $4 \mathrm{~d}^{10}(5 \mathrm{~g}, 6 \mathrm{~g}$ and $7 \mathrm{~g}){ }^{2} \mathrm{G}_{7 / 2,9 / 2}$ levels confirms the correctness of the $4 \mathrm{~d}^{10} 4 \mathrm{f}$ levels. The latter were compiled in AEL [5] and were later confirmed by Bhatia [6]. The $4 \mathrm{~d}^{10} 5 \mathrm{~d}-4 \mathrm{~d}^{10} 5 \mathrm{f}$ transitions lie in our wavelength region. We observed a pair of lines from $4 d^{10} 5 d^{2} D_{3 / 2}$ and ${ }^{2} D_{5 / 2}$, and two transitions from $4 d^{9} 5 s^{2}{ }^{2} D_{5 / 2,3 / 2}$, thus confirming $4 d^{10} 5 f^{2} F_{5 / 2}$. The other level $4 d^{10} 5 f^{2} F_{7 / 2}$ is also expected to gives two transitions, one from $4 \mathrm{~d}^{10} 5 \mathrm{~d}^{2} \mathrm{D}_{5 / 2}$ and the other from $4 \mathrm{~d}^{9} 5 \mathrm{~s}^{22} \mathrm{D}_{5 / 2}$; both were in fact found. Furthermore, the level positions agree well with theoretical prediction of an inverted doublet. The $4 \mathrm{~d}^{10} 6 \mathrm{f}^{2} \mathrm{~F}_{5 / 2,7 / 2}$ levels are strongly mixed with the $4 \mathrm{~d}^{9} 5 \mathrm{~s} 5 \mathrm{p}{ }^{2} \mathrm{~F}_{5 / 2,7 / 2}$ levels. Bhatia [6] reported only the $4 \mathrm{~d}^{10} 6 \mathrm{f}^{2} \mathrm{~F}_{5 / 2}$ level at $198,499.3 \mathrm{~cm}^{-1}$, but our least squares fitted calculation predicted at 191,337 $\mathrm{cm}^{-1}$. This large deviation does not seem to be right. Bhatia [6] reported unresolved $4 \mathrm{~d}^{10} 7 \mathrm{f}$ levels, but we did not find his identified lines on our line list. Therefore, his $4 \mathrm{~d}^{10} 6 \mathrm{f}$ and $4 \mathrm{~d}^{10} 7 \mathrm{f}$ levels could not be confirmed.

Neither the $4 \mathrm{~d}^{10} 4 \mathrm{f}-4 \mathrm{~d}^{10} n \mathrm{~g}(n=5-7)$ nor $4 \mathrm{~d}^{10} 5 \mathrm{~g}-4 \mathrm{~d}^{10} n \mathrm{~h}(n=7-9)$ transitions lie in our wavelength region. Therefore, they could not be confirmed in the present work. However, we have compared Bhatia's experimental results [6] with theoretical calculations for the known spectra in the isoelectronic sequence from Ag I-Sn IV [11], and they appear to be regular. The $4 \mathrm{~d}^{10} 4 \mathrm{f}-(8 \mathrm{~g}+9 \mathrm{~g})$ transitions do lie in our wavelength region, but they are too weak to be verified. However, we have included them in our LSF calculations for the sake of completeness. 


\subsection{The $4 d^{9} 5 s 5 p$ Configuration}

This configuration arises due to core excitation of the ground level configuration $4 \mathrm{~d}^{10} 5 \mathrm{~s}$. A number of levels from this configuration were reported by Bhatia [6]. Kaufman et al. [7] revised three levels of this configuration by observing transitions from the ground level $4 d^{10} 5 s^{2} S_{1 / 2}$, thus connecting only $J=1 / 2$ and $3 / 2$ levels. The remaining levels of Bhatia (with $J=5 / 2,7 / 2$ and 9/2) still remain to be verified. In the present investigation, we agreed with six levels of Kaufman et al. [7] but revised four levels. The ionization separation on our recorded spectrum in this wavelength region was quite clear, thus new levels could be found with full confidence. The level ${ }^{2} \mathrm{P}_{1 / 2}$ reported by Bhatia [6] at $199,561.2 \mathrm{~cm}^{-1}$ was revised by Kaufman et al. [7] to a new position at $197,081 \mathrm{~cm}^{-1}$. The line (507.406 ̊) used by Kaufman et al. [7] for this transition actually belongs to O III (507.391 $\AA$ ) [11] and the line used by Bhatia was not found on our spectrograms. We found an unclassified In III line with moderate intensity at $504.080 \AA$ that has been assigned to this transition, yielding the level value at $198,382.2 \mathrm{~cm}^{-1}$ that also fits well in the least squares calculations.

Kaufman et al. [7] had revised another $J=1 / 2$ level of Bhatia [6] and re-designated it as a $J=3 / 2$ level at $170,888 \mathrm{~cm}^{-1}$ based on Bhatia's line list as they did not observe the corresponding lines. We also could not find the lines associated with this level in our line list. Therefore, this level was rejected. According to our analysis, we found that the lowest $J=1 / 2$ level reported by Kaufman et al. [7] at $170,812 \mathrm{~cm}^{-1}$ is in fact a $J=3 / 2$ level and the replacement for the lowest $J=1 / 2$ level is found at $171,315.7 \mathrm{~cm}^{-1}$. The lowest $J=3 / 2$ level of this configuration reported by Kaufman et al. [7] at $167,079 \mathrm{~cm}^{-1}$ is in fact based on an In IV line (598.526 $\AA$ ) [16,17]. However, Bhatia [6] had reported this level at $167,339.1 \mathrm{~cm}^{-1}$, which was based on a correct In III line at (597.589 $\AA$ ), and we agree with this identification. Moreover, it also gives two transitions from the recently found $4 \mathrm{~d}^{9} 5 \mathrm{p}^{2}$ configuration [9] that confirm the identification of this level.

The highest $\mathrm{J}=3 / 2$ level was not found by Kaufman et al. [7] because calculations predict a weak transition to the ground level. However, Bhatia [6] had reported this level at 202,132.3 $\mathrm{cm}^{-1}$. We found two strong lines with correct In III ionization characteristics, which we classified as transitions from $4 d^{9} 5 s^{2}$ levels to the level in question. Thus, we confirmed Bhatia's level value. Table 4 shows the summary of the $J=1 / 2$ and $3 / 2$ levels of the $4 d^{9} 5 s 5 p$ configuration given by previous researchers [6,7] and the present analysis.

Table 4. Energy level values $\left(J=1 / 2 \& 3 / 2\right.$ Levels) of $4 d^{9} 5$ s5p Configuration.

\begin{tabular}{ccccc}
\hline Configuration $\left(\mathbf{4 d}^{\mathbf{9}} \mathbf{5 s 5 p )}\right.$ & \multicolumn{2}{c}{ Previous Work } & \multicolumn{2}{c}{ This Work } \\
\hline & Bhatia [6] & Kaufman et al. [7] & & \\
$\left({ }^{3} \mathrm{D}\right)^{4} \mathrm{P}_{1 / 2}$ & $170,888.3$ & 170,812 & $171,315.7$ & Revised \\
$\left({ }^{1} \mathrm{D}\right)^{2} \mathrm{P}_{1 / 2}$ & $178,187.5$ & 178,187 & $178,187.72$ & Verified \\
$\left({ }^{3} \mathrm{D}\right)^{4} \mathrm{D}_{1 / 2}$ & $179,321.0$ & 179,321 & $179,320.9$ & Verified \\
$\left({ }^{3} \mathrm{D}\right)^{2} \mathrm{P}_{1 / 2}$ & $199,561.2$ & 197,081 & $198,382.2$ & Revised \\
$\left({ }^{3} \mathrm{D}\right)^{4} \mathrm{P}_{3 / 2}$ & $167,339.1$ & 167,079 & $167,339.24$ & Revised \\
$\left({ }^{3} \mathrm{D}\right)^{4} \mathrm{~F}_{3 / 2}$ & $170,918.9$ & 170,888 & $170,813.7$ & Revised \\
$\left({ }^{1} \mathrm{D}\right)^{2} \mathrm{D}_{3 / 2}$ & $175,538.7$ & 175,538 & $175,539.35$ & Verified \\
$\left({ }^{1} \mathrm{D}\right)^{2} \mathrm{P}_{3 / 2}$ & $178,616.5$ & 178,616 & $178,616.85$ & Verified \\
$\left({ }^{3} \mathrm{D}\right)^{4} \mathrm{D}_{3 / 2}$ & $180,945.0$ & 180,945 & $180,943.95$ & Verified \\
$\left({ }^{3} \mathrm{D}\right)^{2} \mathrm{P}_{3 / 2}$ & $191,509.2$ & 191,508 & $191,509.1$ & Verified \\
$\left({ }^{3} \mathrm{D}\right)^{2} \mathrm{D}_{3 / 2}$ & $202,132.3$ & - & $202,134.5$ & Verified \\
\hline
\end{tabular}

The remaining 12 levels of this configuration with higher $J$ values (5/2-9/2) were considered next. These levels have only been reported by Bhatia [6] through the transitions from $4 d^{9} 5 s^{2}$. We found lines corresponding to transitions from the $J=5 / 2$ level at $194,902.6 \mathrm{~cm}^{-1}$ and confirmed only this level in Bhatia's list. We were successful in locating 10 remaining levels of $J=5 / 2$ and $7 / 2$ from transitions to $4 d^{9} 5 s^{2}$ and $4 d^{9} 5 p^{2}$ levels. The level with the highest $J$ value $(9 / 2)$ does not connect to any other known configuration except $4 \mathrm{~d}^{9} 5 \mathrm{p}^{2}$, which was partially known. We extended that configuration to 
include $J=7 / 2$ levels. This paved the way for the establishment of the $J=9 / 2$ level. We found three transitions placing the $J=9 / 2$ level at $168,947.6 \mathrm{~cm}^{-1}$. All 23 levels of $4 \mathrm{~d}^{9} 5 \mathrm{~s} 5 \mathrm{p}$ configuration are now known experimentally.

\subsection{The $4 d^{9} 5 s(n f+n p)$ Configurations}

These are the configurations that arise due to the core excitation. The $4 \mathrm{~d}^{9} 5 \mathrm{~s} 4 \mathrm{f}$ configuration has a large energy spread and contains 39 levels. Since the ground configuration contains only the ${ }^{2} S_{1 / 2}$ level, only $J=1 / 2$ and $3 / 2$ levels of the $4 \mathrm{~d}^{9} 5 \mathrm{~s} 4 \mathrm{f}$ configuration can decay to the ground configuration. Kilbane et al. [8] have studied the $4 \mathrm{~d}^{9} 5 \mathrm{~s} n \mathrm{f}(n=4-12)$ and $4 \mathrm{~d}^{9} 5 \mathrm{~s} n \mathrm{p}(n=6-11)$ configurations using a photoabsorption technique. They reported 10 levels of $4 d^{9} 5 \mathrm{~s} 4 \mathrm{f}$ and seven levels of $4 \mathrm{~d}^{9} 5 \mathrm{~s} 6 \mathrm{p}$ belonging to $J=1 / 2$ and $3 / 2$. In our spectra, these transitions lie in the shorter wavelength region, where reflectivity of the grating falls considerably in the normal incidence setting. Therefore, these transitions appeared with very weak intensity on our spectrograms. Secondly, a large number of In V [18] and In VI [19] transitions overlap in this region. Therefore, it was very difficult to identify confidently In III lines of this array. Moreover, these levels lie above the ionization limit and consequently have a very small population. Therefore, these levels could not be located in the present work. However, we performed least squares fitted parametric calculations to provide a precise prediction of the remaining levels of the $4 \mathrm{~d}^{9} 5 \mathrm{~s} n \mathrm{f}(n=4-7)$ and $4 \mathrm{~d}^{9} 5 \mathrm{~s} n \mathrm{p}(n=6-7)$ configurations based on the identification made in reference [8].

\subsection{The $4 d^{9} 5 p^{2}$ Configuration}

The first attempt to study the low-lying autoionizing configuration $4 \mathrm{~d}^{9} 5 \mathrm{p}^{2}$ in the sequence In III-Te VI was made by Ryabtsev et al. [9], connecting this configuration with $4 \mathrm{~d}^{10} 5 \mathrm{p}$. It is important to note that all the levels of this configuration lie above the ionization limit. It was difficult to arrange experimental conditions providing for a reasonable population above the ionization limit. Certainly it was advantageous to identify the broad lines due to continuum effect, but only the strongest transitions could be observed. Not many pairs connecting to both $4 d^{10} 5 p^{2} P_{1 / 2,3 / 2}$ were found to confirm these levels. However, the lines used to locate these levels have a definite In III characteristic and show continuum broadening effect. Out of 28 levels of $4 \mathrm{~d}^{9} 5 \mathrm{p}^{2}$, only 13 levels with $J=1 / 2,3 / 2$ and $5 / 2$ were reported by Ryabtsev et al. [9]. We should point out that two levels $\left({ }^{1} \mathrm{D}\right){ }^{2} \mathrm{~S}_{1 / 2}$ and $\left({ }^{1} \mathrm{D}\right){ }^{2} \mathrm{P}_{3 / 2}$ were reported by Ryabtsev et al. [9] with the same energy level values. They were based on the double classification of the same pair of lines (555.669 $\AA$ and $569.421 \AA$ ). We agreed with assignments of these lines to $\left({ }^{1} \mathrm{D}\right){ }^{2} \mathrm{P}_{3 / 2}$ giving the level value at $237,145.6 \mathrm{~cm}^{-1}$ as both transitions are predicted to be of the comparable intensity. However, the $\left({ }^{1} \mathrm{D}\right){ }^{2} \mathrm{~S}_{1 / 2}$ level is predicted to have one strong and one weak transition, and we found one unclassified line on our plate at $555.501 \AA$, which we used to establish this level at $237,201.7 \mathrm{~cm}^{-1}$. Several levels have also been confirmed through transitions to the $4 d^{9} 5 s 5 p$ configuration. The higher $J$ values of $4 d^{9} 5 p^{2}$ configuration $(J=7 / 2$ and $9 / 2)$ could only be established through transitions from $4 \mathrm{~d}^{9} 5 \mathrm{~s} 5 \mathrm{p}$. We were successful in establishing three $J=7 / 2$ and one $J=9 / 2$ levels. One $J=9 / 2$ and two $J=7 / 2$ levels remain unknown. The study of the $4 \mathrm{~d}^{9} 5 \mathrm{p}^{2}$ and $4 \mathrm{~d}^{9} 5 \mathrm{~s} 5 \mathrm{p}$ configurations together complemented each other. The other even parity configuration $4 d^{9} 5 s 5 d$ lies above the ionization limit and partially overlaps the $4 d^{9} 5 p^{2}$ configuration. It has also been incorporated in the least squares fitted parametric calculation to interpret the results.

\section{Optimization of the Energy Levels}

The transition wavelengths observed for this spectrum were used to derive the energy level values. For this purpose, a least-squares level optimization code LOPT [15] was used. The essential factors for the level optimization procedure are the correct identification of the spectral lines and estimation of their uncertainties. The wavelength uncertainty is determined by the combined effect of the statistical deviation of the line position measured on the comparator and systematic uncertainty of reference wavelengths used in the fitting. Ryabtsev et al. [9] reported the uncertainty of autoionized 
lines to be $\pm 0.006 \AA$. Our wavelength accuracy for sharp and unblended lines is estimated to be within $\pm 0.006 \AA$ and $\pm 0.008 \AA$ below and above $900 \AA$. We estimated the uncertainty of Bhatia's lines to be $\pm 0.008 \AA$ for lines below $2000 \AA$ with the comparison of our measurement and Kaufman et al. [7] for sharp and unblended lines. Bhatia mentioned in his paper that the prism lines are not accurate to more than $0.01 \AA$. However, he gave wavelengths above $2000 \AA$ with only two places after the decimal point implying that the uncertainty is at least $0.02 \AA$ or higher. In our level optimization with Bhatia's lines [6], we noticed several lines showing a deviation around $0.22 \AA$ for the region $2000-4000 \AA$ from their Ritz values. The deviation increases up to $0.8 \AA$ for the longer-wavelength region $4000-6500 \AA$. We, therefore, did not use these lines with large deviation in the level optimization. All of the lines used in the optimization of the level values were given an estimated uncertainty to find the final optimized energy level values with an estimated uncertainty for each level. Since the level $4 d^{10} 5 p\left({ }^{1} S\right)$ ${ }^{2} \mathrm{P}_{3 / 2}$ connects with the largest number of observed transitions, it was adopted as the base level, hence all the level uncertainties in Table 2 are given with respect to this level. All the given uncertainties are taken to be at the level of one standard deviation.

\section{Ionization Potential}

Since more than one series with three members are known in In III, its ionization potential can be determined with good accuracy. The value of ionization potential of In III given in AEL [5] at $226,100 \mathrm{~cm}^{-1}$ was derived by Catalan and Rico [20] by comparison of the third spectra from $Y$ to In. Bhatia [6] improved the value of ionization potential by using $4 \mathrm{~d}^{10} n \mathrm{~g}(n=5-9)$ and $4 \mathrm{~d}^{10} n \mathrm{~h}(n=6-9)$ in frames of the polarization theory [21]. He calculated the In III limit at $226,191 \mathrm{~cm}^{-1}$; this value is listed in the NIST Atomic Spectra Database [11]. We have calculated the ionization potential from two series, $n$ s $(n=5-12)$ and $n g(n=5-9)$ using the Ritz quantum defect extrapolation method with the aid of the RITZPL code [22]. However, the non-penetrating $\left(4 \mathrm{~d}^{10} n \mathrm{~g}\right)$ series is certainly expected to give more accurate value. The value of IP obtained using the three-parameter extended Ritz formula [22] for the $4 \mathrm{~d}^{10} n \mathrm{~s}(n=5-12)$ series is $226,196.58 \mathrm{~cm}^{-1}$, while the values obtained by fitting the two-parameter extended Ritz formula for the two $4 \mathrm{~d}^{10} \mathrm{ng}^{2} \mathrm{G}_{7 / 2,9 / 2}(n=5-9)$ series are 226,197.00 $\mathrm{cm}^{-1}$ and $226,195.08 \mathrm{~cm}^{-1}$, respectively. The limits calculated by the POLAR code [22] for the $n \mathrm{~g}(n=5-9)$ ${ }^{2} \mathrm{G}_{7 / 2}, 9 / 2$ series were found to be $226,197.28$ and $226,195.35 \mathrm{~cm}^{-1}$, respectively. The adopted value is the average of these calculations at $226,196.3 \mathrm{~cm}^{-1} \pm 1.0 \mathrm{~cm}^{-1}(28.0448 \pm 0.0001 \mathrm{eV})$ differing by $5 \mathrm{~cm}^{-1}$ from Bhatia's value.

\section{Conclusions}

A total of 91 energy levels have been established, among which three levels are revised and 21 are new. All of these levels were based on the identification of 218 spectral transitions, 70 being new. The results were interpreted using Cowan's codes and the least square fitted parametric theory. The optimized energy levels and their calculated values are given in Table 2 along with the level uncertainty, LS-percentage compositions and number of connecting transitions. All of the classified transitions are given in Table 3 along with their weighted transition probabilities $(g A)$ obtained with least squares fitted energy parameters. This table also contains the Ritz wavelengths of all transitions with their uncertainties obtained by using the level optimization code (LOPT).

Author Contributions: All authors contributed equally to this work.

Conflicts of Interest: The authors declare no conflict of interest.

\section{References}

1. Rao, K.R. On the Spectra of Doubly-Ionised Gallium and Indium. Proc. Phys. Soc. 1927, 39, 150-160. [CrossRef]

2. Lang, R.J. On the Spectra of Zn II, Cd II, In III and Sn IV. Proc. Natl. Acad. Sci. USA 1929, 15, 414-418. [CrossRef] [PubMed] 
3. Archer, D.H. The Spectra of Indium. Master's Thesis, University of British Columbia, Vancouve, BC, Canada, 1948.

4. Nodwell, R.A. A Study of Spark Spectrum of Indium. Ph.D. Thesis, University of British Columbia, Vancouve, BC, Canada, 1956.

5. Moore, C.E. Atomic Energy Levels, National Bureau of Standards Circular 467; US Govt. Printing Office: Washington, DC, USA, 1958; Volume III.

6. Bhatia, K.S. Spectrum of Doubly Ionised Indium. J. Phys. B At. Mol. Phys. 1978, 11, 2421-2434. [CrossRef]

7. Kaufman, V.; Sugar, J.; VanKleef, T.A.M.; Joshi, Y.N. Resonance transition $4 d^{10} 5 \mathrm{~s}-4 \mathrm{~d}^{9} 5 \mathrm{~s} 5 \mathrm{p}$ in the Ag I sequence of In III, Sn IV, Sb V, and Te VI. J. Opt. Soc. Am. B 1985, 2, 426-429. [CrossRef]

8. Kilbane, D.; Mosnier, J.-P.; Kennedy, E.T.; Costello, J.T.; van Kampen, P. 4d Photoabsorption Spectra of Indium (In II-In IV). J. Opt. Soc. Am. B Opt. Phys. 2006, 39, 773-782. [CrossRef]

9. Ryabtsev, A.; Churilov, S.S.; Kononov, É.Y. $4 \mathrm{~d}^{9} 5 \mathrm{p}^{2}$ Configuration in the Spectra of In III-Te VI. Opt. Spectrosc. 2007, 102, 354-362. [CrossRef]

10. Skočić, M.; Burger, M.; Bukvić, S.; Djeniže, S. Line intensity and broadening in the In III spectrum. J. Phys. B At. Mol. Opt. Phys. 2012, 45, 225701. [CrossRef]

11. Kramida, A.; Ralchenko, Y.; Reader, J.; NIST ASD Team. NIST Atomic Spectra Database, v. 5.4, National Institute of Standards and Technology, Gaithersburg, MD, USA. 2016. Available online: http:/ / physics.nist. gov / ASD (access on 7 June 2017).

12. Cowan, R.D. The Theory of Atomic Structure and Spectra; University California Press: Berkeley, CA, USA, 1981.

13. Kramida, A. Critically Evaluated Energy Levels and Spectral Lines of Singly Ionized Indium (In II). J. Res. Natl. Inst. Tech. 2013, 118, 52-104. [CrossRef] [PubMed]

14. van het Hof, G.J. A Computer Program-FIND3, for Searching the Levels; Zeeman Lab: Amsterdam, The Netherland, 1994.

15. Kramida, A.E. The program LOPT for least-squares optimization of energy levels. Comput. Phys. Commun. 2011, 182, 419-434. [CrossRef]

16. Ryabtsev, A.N.; Kononov, E.Y. High Lying Configurations in the Spectrum of Three Times Ionized Indium (In IV). J. Quant. Spectrosc. Radiat. Transf. 2016, 168, 89-101. [CrossRef]

17. Swapnil; Tauheed, A. Revised and Extended Analysis of the Fourth Spectrum of Indium: In IV. J. Quant. Spectrosc. Radiat. Transf. 2013, 129, 31-47. [CrossRef]

18. Joshi, Y.N.; VanKleef, T.A.M.; Kushawaha, V.S. The Fifth Spectrum of Indium: In V. Can. J. Phys. 1976, 54, 889-894. [CrossRef]

19. Joshi, Y.N.; VanKleef, T.A.M. Sixth Spectrum of Indium: In VI. J. Opt. Soc. Am. 1982, 72, 259-267. [CrossRef]

20. Catalán, M.A.; Rico, F.R. Series y potenciales de ionizacion en los espectros III de los elementos del grupo del paladio. An. Fis. Quim. Ser. A 1957, 53, 85.

21. Edlen, B. Wavelength measurements in the vacuum ultra-violet. Rep. Prog. Phys. 1963, 26, 181. [CrossRef]

22. Sansonetti, C.J. (National Institute of Standards and Technology, Gaithersburg, ML, USA). Computer Programs RITZPL and POLAR. Private Communication, 2005.

(C) 2017 by the authors. Licensee MDPI, Basel, Switzerland. This article is an open access article distributed under the terms and conditions of the Creative Commons Attribution (CC BY) license (http:/ / creativecommons.org/licenses/by/4.0/). 